\title{
Topics in Low-Dimensional Field Theory •
}

\author{
Michael J. Crescimanno \\ Theoretical Physics Croup \\ Physics Division \\ Lawrence Berkeley Laboratory \\ 1 Cyclotron Road \\ Berkeley, California 94720
}

\begin{abstract}
Abstraci
Conformal field theory is a natural tool for understanding two-dimensional critical systems. This work presents results in the lagrangian approash to conformal field theory. The first sections are chiefly about a particular class of field theories called coset constrctions and the last part is an exposition of the connection between two-dimensioal conformal theory and a three-dimenional gauge theory whose lagrangian is the Chern-Sicsons density.
\end{abstract}

This report has been reproduced directly from the best available copy.

-This work was supported by the Director, Office of Energy Research, Oftice of High Energy and Nuclear Physics, Division of High Energy Physics of the U.S. Department of Energy under Contract DE-AC03-76SF00098. 


\section{TABLE OF CONTENTS}

1. Introduction

2. Monopole Backgrounds on the World Sheet

3. Parafermions and Coset Models

(a) The Abelian Coset Model

(b) The Non-Abelian Coset Model

4. Classical W-Algebras and Non-Abelian Coset Models

5. Chern-Simons Quantum Mechanics, Modular Invariance and Monopoles

6. Conciusion 


\section{CHAFTER 1: Introduction}

Since 1984 there has been strong renewed interest in field theory in two and three dimensions. In that year Green and Schwarz discovered an anomaly-free string theory' thereby launchiag hopes that there might be a unique, finite fundamental theory of gauge interactions and gravity. In the intervening years although the prospect of understanding physical interactions in terms of string theory have dimmed there have been vigorous efforts and inroads made in understanding aspects of low-dimensional field theory.

String theory is an old subject that was first motivated by an attempt to understand the strong interactions ${ }^{2}$. In particular it was discovered ${ }^{3}$ that, in the large $N$ limit of the gauge theory $\mathrm{SC}(\mathrm{N})$, the dominant contributions from perturbation theory come from planar diagrams. This combined with earlier work 4 suggested that one may think of mesons. hadrons and other strongly interacting particles as being essentially quarks tied together by tubes of strongly interacting gauge bosons.

This idea was further expanded upon in ref.[5] in which a quantum mechanical theory of such an extended particle was described in terms of a lagrangian that measured the area of the surface swept out by the tubes as they propagate in space. It was thus discovered that because the area of the sheet swept out by the string didn't depend on the co-ordinates used to label the points in the sheet, that the resulting theory had a two-dimensional conformal invariance. The picture is as follows; as the string propagate in some background space. the functions that describe how the string is situated in space at every instant (called the embedding fields) if viewed in terms of the two-dimensional co-ordinates oi the sheet. become fields of a conformal field theory. In two dimensions the conformal group is infinitedimensional and its representations have been studied in ref.[6].

Two-dimensional systems that possess conformal symmetries are of interest for reasons other than string theory. Indeed, any two-dimensicnal system undergoing a second order phase transition will be describable at the critical point in terms of a conformal field 
theory. Although there are very stringent conditions for the existence of phase transitions in systems of such low dimensionality there are $\mathbf{2}$ wealth of examples provided by the known conformal field theories. For more on the connection between coiformal invariance and statistical mechanics see ref.[r]. Thus, conformal field theory is a subject that joins string theory, statistical mechanics and the representation theory of infinite-dimensionad lie algebras in an unusual harmony.

Conformal field theory has also lead to a surge of interest in possibly understanding theories of gravitation. In particular it was known fron the early days of string theory that any string theory necessarily contains states of arbitrarily high spin and in the simplest consistent string theories that were written down there appeared massless spin one and spin two fields ${ }^{8}$. The massless spin one fields were thought of as gauge bosons and the massless spin two field was to be identified with the graviton. Indeed, it was known that any interacting massless spin two excitation must contribute to the Einstein-Hilbert action and therefore may be thought of as a type of graviton'. In addition to finding these massless states the string aiso had excitations that had negative mass-squared. These states, called tachyons because their dispersion relation indicates that they travel faster than the speed of light, are not permisable on physical grounds. Furthermore, the early string theorists were interested in a theory of strong interactions and so didn't want a theory with either massless spin two fields or tachyons. Later it was found that in the supersymmetric generailzation of string theory there are no tachyon states ${ }^{10}$. Although this theory still had a massless spin two field, many physicists began to regard this theory as a toy model for a theory with both gravitation and gauge interactions.

Indeed, gravity arises in a very natural way in (closed) string theory. As remarked earlier, one may think about the string propagating in some background spacetime in terms of constrained embedding fields. These fields become those of a two dimensional field theory. Such a system has been analyzed in detail in ref.[11] in which it is shown that consistency of such a model (at the level of quantum mechanics) requires the background spacetime to be a solution of the free space Einstein equation. This is a very stringent condition and 
may be understood from many points of view.

Trying to understand gravity as a quantum theory has been a hallmark of string theory. Although two dimensional gravity is trivial (in the sense that the equations of motion are satisfied identically) conformal invariance is part of the classical invariance of a two dimensional gravity theory. Understanding how to represent this classical invariance as unitary transformations on the Hilbert space of the theory gives one a sense of what a quantum theory of gravity might look like. One conventionally thinks of only metrical theories of gravity but it has been pointed out in ref.[12] that if one imagines including in the path integral contributions from metrics that are singular and of arbitrary signature (called studying gravity in the "unbroken" phase to draw analogy with the phases of the $\phi^{4}$ model) one may rewrite three dimensional gravity as a topological theory. By a topological theory we understand a field theory that does not involve the background metric. Thus integrating over the metrics (as one is instructed to do in a path integral of gravity) is trivial. This idea had its antecedent in understanding three dimensional topological gauge theories ${ }^{13}$. One particularly interesting facet of this approach is that it allows one to understand conformal field theories from another point of view, namely the conformal invariance is a residual symmetry of the three dimensional general co-ordinate invariance. There is still much to be done to understand more precisely the connection between the three dimensional topological field theories and two dimensional conformal field theories.

This thesis contains work done over the last few years that pertains to the above discussed topics. Since most of this work has already been published an effort has been made to preserve the form in which the work appeared. For each paper the title page has been included because of joint authorship. Chapter I contains the paper "Monopoles on the World Sheet" in which the effect of including non-trivial gauge backgrounds in the path integral of a simple coset model is investigated. Coset models are a particularly ubiquitous type of conformal field theory and so the phenomena remarked upon here are presumed to bo of a general nature. Chapter II contains two monographs on computing the Poisson brackit of coset models, the first one for the case of abelian cosets and the second one for non- 
abelian cosets. The results of this investigation compare with the classical limit of certain known theories and really provides a unifying framework for semiclassically understanding these theories. The Poisson bracket of chapter II is then used in a short note in chapter III to show how one may understand the classical limit of W algebres. Finally, chapter $N$ contains a paper which explores the connection between topological field theory in tbree dimensions and conformal field theory in two dimensions. Chapter $V$ contains a conciusion and this authors' outlook. 


\section{Bibliography}

1. M.B. Green and J.H. Schwarz, Phys. Lett. B149, 11 (1984).

2. G. Veneziano, Phys. Reports C9, 199 (1974),

3. G. $t$ 'Hooft, Nucl. Phys. B72, 461 (1974).

4. H.B. Nielsen and P. Olesen. Phys. Lett. B32, 203 (1970).

5. A.M. Polyakov, Phys. Lett. B103, 207 (1981).

6. A.A. Belavin, A.M. Polyakov and A.B. Zamalodchikov, Nucl. Phys. B241, 333 (1984).

i. J. Cardy, Sicl. Plyss. B270. 186 (1986).

8. S. Fubini and G. Veneziano, Ann. Phys. 63, 12 (19i1).

9. D. Bouware and S. Deser, Ann. Phys. 89, 193 (19i5).

10. J.H. Schwarz, Phys. Reports 89, 223 (1982).

11. D. Friedan, Ann. Phys. 163, 318 (1985).

12. E. Witten, Nucl. Phys. 311, 46 (1988).

13. E. Witten, Comm. Math. Phys. 121, 351 (1989). 


\section{ACKNOWLEDGEMENTS}

The author would like to thank his advisor Professor $\mathrm{K}$. Bardakci for his insight, intuition and experience. Without his guidance and gentle-spirited approach these years of graduate study would likely have been unsewarding. The author wishes to thank S.A. Hotes for perseverance and patience in working together on many things. Tharks also to A. Giveon, E. Kiritsis and D.J. Smit for conversations and explainations and to Professors O. Alvarez, M.B. Halpern. H. Morrison and B. Zumino of the Physics Department and Professor V.F.R. Jones of the Mathematics Department and Professor E. Rabinovici (of the Fiebrew University; Jerusalem) for many explanations. It is a pleasure to acknowledge useful incidental conversations with I. Bars (UCLA), D. Bar-Natan (Princeton), M. Bos (Columbia), K. Intrilligator (Hariard), S. Naculich (John Hopkins University), V.P. Nair (Columbia), and A. Polychronakos (Florida State). For innummerable technical discussions on a variety of topics this author wishes to thank A. Landsberg, S. Hsu and N. Obers. Special thanks to A. Landsberg. T. Pipp,S.J. and M.K. Cohan for their warm friendship and support. 


\section{CHAPTER 2: Monopole Backgrounds on the World Sheet}

Since the advent of string theory it has been usefull to thing about the string propagating in some geometrically fixed, i.e. classical, background. In this first paper we investigate what effect a monopole background will have on a siring. As described in the introduction to this work, this is an iateresting question because a broad class of models (the so called coset constructions) for the compactification of the theory involve integrals over a non-dynamical gauge field. Thus it is natural to ask what may be learned about the theory by studying it in non-trivial gauge backgrounds.

The entire paper is a description of a very particular type of coset i.e. those cosets that may be realized in terms of free fermions coupled to an abelian gauge field (as an example consider $S O(2 n) / S O(2)$ cosets). Highlights include a new spectrum for states propagating in this monopole background and also novel modular properties at genus one. 
Monopole Backgrounds on the World Sheet

\author{
K. Bardakci \\ and \\ M. Crescimanno \\ Department of Physics \\ Cniversity of California \\ Berkeley, California 94720 \\ and \\ Laurence Berieley Laboratory
}

appeared in Nuclear Physics B, Vol. 313 (1989), pg. 269 


\section{Introduction}

Compactification has emerged as one of the central problems of string theory. ${ }^{1-8}$ Vari. ous compactification schemes have already led to the construction of semi-realistic models. The simplest version, the torojdal compactification, can be described either in the language of bosons or of fermions on the world sheet. ${ }^{9-13}$ These models have, in genecal a built-in $S O(N)$ Kac-Moody algebra. The next simplest version of compactification makes ure of the coset construction. ${ }^{14}$ By projecting out a suitable subgroup of the initial compactification group, it is possible to construct various non-trivial models, including the whole series with central charge less tilan one. ${ }^{14}$ This projection can be carried out implicitly by coupling suitable currents of the free fermion model to gauge ficlds without kinetic energ terms. These gauge fields then act as lagrange multipliers and set the corresponding currents equal to zero. $8,15,16,17$

In this paper, we investigate the possibility that the gauge fields used in the coset construction have non-trivial topological structure. The simplest possibility is an abeliar. gauge field with non-vanishing total flux over a compact surface; the total flux is then quantized in integer multiples of fundamental monopole charge. (For an excellent review, see ref.[20]). In section 2, we consider the model where the $U(1)$ current of free fermions with a flavor quantum number is coupled to a gauge field. We allow the gauge field to carry non-trivial monopole charge by introducing a "classical" background field with non-trivial topology. In the next section, correlation functions of gauge invariant fertsionic bilinears are explicitly caisulated by carrying out the summation over backgrounds of different monopole charge. The result of this two dimensional "instanton" calculation is similar to the four dimensional one: invariance under chiral rotations is spontaneously broken. We have a'so factorized the correlation functions and study the resulting spectrum. In section 4 we investigate the modular invariance of the model by studying the zero mode solutions on 
the torus. Although we prestnt som; unusual features, the standard sum over tise four spin structures is still modular invariant. Finally, in the last section, ve summurize our conclusicans and discuss pussible extensions. In the appendix that follows, the analysis of modular invariance is extended to non-zero modes. 


\section{The Model.}

We start with $\kappa$ Dirac fermions in two dimensions, coupled to an abelian gauge field $A_{\mu}$ that serves as a Lagrange multiplier. The action is given by

$$
\begin{aligned}
& A=\int d^{2} x \mathcal{L} \\
& \mathcal{L}=\sum_{\alpha=1}^{\infty} \psi^{\alpha}(i \rho+A) \psi^{\alpha},
\end{aligned}
$$

where the metric is Euclidean aid the two dimensional gamma matrices are hermitian. Integration over the gauge field imposes the constraint that the current must vanish

$$
j_{\mu}=\sum_{a} \psi^{a} \gamma_{\mu} \psi^{a}=0
$$

Since this constraint is awiward to implement directly, it is customary to first eliminate the gauge field by a redefinition of the fermion fields. Consider taking the gauge

$$
\partial_{\mu} A_{\mu}=0
$$

which allows one to set

$$
A_{\mu}=\epsilon_{\mu \nu} \quad \partial_{\nu} \phi
$$

where $\phi$ is a scalar field which can be absorbed by a redefinition of the fermions:

$$
\psi^{\circ} \rightarrow \exp \left(\gamma_{3} \phi\right) \psi^{\alpha}, \quad \bar{\psi}^{\alpha} \rightarrow \bar{\psi}^{\circ} \exp \left(\gamma_{3} \phi\right),
$$

with $\gamma_{3}=i \gamma_{1} \gamma_{2}$. However, by virtue of the anomaly equation

$$
\partial_{\omega, 3}=-\frac{\kappa}{\pi} F
$$

where,

$$
\begin{aligned}
j_{\mu, 3} & =i \sum_{-\sigma} \bar{\psi}^{\alpha} \gamma_{\mu} \gamma_{3} \psi^{\alpha}=\epsilon_{\mu \nu} j_{\nu,} \\
F & =\varepsilon_{\mu \nu} \partial_{\mu} A_{\nu},
\end{aligned}
$$


this redefinition induces an extra term in the action:

$$
\Delta \mathcal{C}=-\frac{\kappa}{2 \pi}\left(\partial_{\mu} \phi\right)^{2}
$$

Sr ar, all this is well known; however, here, we wish to generalize the preceding analysis to gauge fields associated with monopoles. Consider a compact surface, $\Sigma$, like a sphere or torus; then it can be shown for arbitrary genus that the gauge configurations satisfying the monopole quantization condition

$$
\int_{\underline{x}} F d^{2} x=2 \pi N
$$

with $N=$ integer, can be included in the functional integral over the gauge fielc $A_{\mu}$. If $N$ is different from zero then it is not possible to globally define an $A_{\mu}$ satisfying eq.(2.i); rather the vector potential will be a nontrivial $U(1)$ bundle.

In particular, the field 0 of eq.(2.3b) cannot be defined globally. To overiome this problem, we replace this equation by

$$
A_{\mu}=A_{\mu}^{c}+\epsilon_{\mu} \partial_{\nu} \dot{\phi}
$$

where $A_{\omega}^{c}$ is a fixed. patch-dependent gauge field that satisfies eq. (2.i), and the field $O$ is now globally defined. One may think of $A_{\mu}^{c}$ as a classical background field that carries the net monopole flux, and $o$ may be thought of as the quantum field (responsible for redistributing the given monopole flux) to be path integrated. This model is still conformally intariant. both classically arid quantum mechanically, since a conformal transformation would change $A_{\mu}^{c}$ only by terns that can be removed by suitable gauge transformations ( $A_{\mu} \rightarrow A_{\mu}+\partial_{\mu} \alpha$, $\alpha$ not respecting $\square \sigma=0$ necessarily) and shifts in $\phi$. Since we wish to perform path integration over $\alpha$ and $\phi$ the model is manifestly quantum mechanically conformally invariant. Taking into account eqs.(2.4), (2.6) and (2.8), the final Lagrangian is

$$
\mathcal{L}_{N}=\sum_{a=1}^{n} \vec{\psi}^{\alpha}\left(i \partial+A^{\epsilon}\right) \psi^{\alpha}-\frac{\kappa}{2 \pi}\left(\partial_{\mu} \phi\right)^{2}-\frac{\kappa}{\pi} \phi F^{\epsilon}
$$


where

$$
F^{c}=\epsilon_{\mu} \partial_{\mu} A_{\nu}^{c}
$$

and $F^{c}$ satisfies the quantization condition eq.(2.7). Actually there is a term missing from the above Lagrangian. This term contains the Faddeev-Popov ghosts from the gauge constraint eq.(2.3a) and it plays an important role if the above model is incorporated into a string compartification scheme. It is, however, not relevant to the developments in this paper and will henceforth be omitted.

The Lagrangian given by eq.(2.9) is conformally invariant, and, as pointed out in the introduction, it can be used for string compactification or in its own right as an example of a ccnformal two-dimensional model. The calculation of the functional integral using the Lagrangian of eq.(2.9) proceeds as in the standard instanton calculations: one has to integrate over the quantum fields for a fixed $A_{\mu}^{c}$, and then sum over different $A_{\mu}^{c}$ corresponding to different $N$ (so long as $A_{\mu}^{c}$ satisfies the conditions eq.(2.7) and eq.(2.3a)).

In the next section, we shall present the details of the calculation of various correlation functions for the model on the plane. Before delving into these computations we briefly review some of the standard instanton lore that is relevant in our case. The most inportane feature of a calculation of the type outlined above is the resulting spontaneous breakdown of invariance under chiral rotations given by

$$
\psi^{\beta} \rightarrow \exp \left(i a \gamma_{3}\right) \psi^{\beta}
$$

As a result, the right and left fermion numbers are no longer separately conserved in the presence of monopoles; only their sum remains a good quantum numbar. The violation of chirality satisfies the following selection rule: If, in a given process, $N_{r}$ and $N_{l}$ ase the number of the right handed and the left handed fermions (of any flavor) respectively; then the amplitude for the process is zonzero only when

$$
N_{r}-N_{\ell}=N_{\kappa},
$$


where $N$ is the monopole number. This selection rule directly follows from the existence of zero mode solutions to the Dirac equation in the presence of a non-trivial background. The index theorem guarantees the existence of $N$ square integrable solutions to the equation

$$
\left(i \not \partial+A^{c}\right) \eta=0 \text {. }
$$

For positive $\boldsymbol{N}$, the olutions are right handed $\left(\gamma_{3} \eta=\eta\right)$, and for negative $N$, they are left handed $\left(\gamma_{3} \eta=-\eta\right)$. Strictly speaking, these statements hold only on a compact surface, and later we will look for the solutions of eq.(2.13) on the plane, supplemented by the point at infinity: For $A_{\mu}^{c}$. we choose.

$$
A_{\mu}^{e}=\epsilon_{\mu \nu} \partial_{\nu}(g)
$$

where $g$ is a snooth function with the following asympiotic behavior:

$$
g_{|x| \rightarrow \infty}-N \log |x|
$$

in order to satisfy' eq. (2.7).

In this fashion a Dirac singularity is introduced at infinity. It is possible to avoid this singularity by considering two overlapping patches, one around the origin and one around infinity, accompanied by two different functions $g^{+}$and $g^{-}$, to be used in eq.(2.14a). One may easily verify that both methods yield identical end results and so for the purpose of exposition we choose to work entirely in one patch thereby putting a Dirac string at infinity.

With the backgtound given by eq.(2.14a), eq. (2.13) splits into two decoupled equations:

$$
\begin{aligned}
& \partial_{z}\left(\eta_{R}\right)-\partial_{x}(g) \eta_{R}=0, \\
& \partial_{x}\left(\eta_{L}\right)+\partial_{x}(g) \eta_{L}=0,
\end{aligned}
$$

where $z=x+i y, \bar{z}=x-i y$. The solutions are given by

$$
\eta_{R}=f_{R}(z) h(z, \bar{z}),
$$




$$
\eta_{L}=f_{L}(\bar{z}) h^{-1}(z, \bar{z})
$$

where $h=\exp (g)$ and $\int_{R . L}$ are entire analytic functions of their respective arguments. By virtue of eq.(2.14), the asymptotic behavior of $h$ is given by

$$
h \underset{|x| \rightarrow \infty}{\longrightarrow}|z|^{-N}
$$

Now, let us require the $\eta$ be square integrable. For positive $N$, only $\eta_{\boldsymbol{A}}$ is square integrable, and the entire function $f_{R}$ has to be a polynomial of maximum degree $N-1$. For negative $N$ the roles of $\eta_{R}$ and $\eta_{L}$ are interchanged. The zero mode solutions are therefore given by the following list:

$$
\eta_{m, R}=z^{m} h(z, \bar{z})
$$

for $y>0, \pi_{m, L}=0$ and

$$
\begin{aligned}
& \eta_{m, A}=0 \\
& \eta_{m, L}=\bar{z}^{m} h^{-1}(z, \bar{z})
\end{aligned}
$$

for $N<0$, where $m=0,1,2, N-1$ throughout.

There is small technical problem related to the question of square integrability on a plane. The flat metric which we have been using so far would eliminate the mode corresponding to $m=N-1$. By mapping the plane onto a suitable compact surface, for example, the sphere, and using the natural metric on this surface, one can easily show that this mode is indeed square integrable. Apart from this single issue, the details of the metric never enter the problem.

So far, we have only considered the integer (periodic in rigid rotations of $2 \pi$ about $z=0$ ) modes. We know from standard string theory that half-integer (antiperiodic) modes are also important. They are projerly defined on a double-cover of the plane, and the 
corresponding wave functions are given by taking $m=-\frac{1}{2}, \frac{1}{2}, \cdots, N-\frac{3}{2}$. These functions will be used in constructing amplitudes in the next section.

As expected for the perjodic modes, the $N$ square integrable solutions saturate the two-dimensional index theorem. Let $n_{R}\left(n_{L}\right)$ be the number of right (left) handed zero modes. The index theorem:

$$
n_{R}-n_{L}=C_{1}(A)=N
$$

is satisfied by the above solutions in eq. $(2.17 \mathrm{a}, \mathrm{b}),\left(C_{1}(A)\right.$ is the first chern invariant for the bundle $A_{\mu}$ ). We may include the antiperiodic modes in our accounting by looking at the double-cover of the sphere in which the total flux (and $C_{1}(A)$ ) will be $2 . N$. 


\section{Correlation Functions on the Plane}

In this section, we sketch the calculation of various correlation functions on the plane and explicitly display some typical ones. We shall consider correlation functions of gauge invariant operators bilinear in the fermion felds. These fall into two classes: The chiral charge conserving operators (cursents) given by

$$
\begin{aligned}
& j_{+}^{\alpha \beta}(z)=\vec{\psi}_{R}^{\alpha}(z) \psi_{L}^{\beta}(z) \rightarrow \vec{\psi}_{R}^{\alpha} \psi_{L}^{\beta}+\frac{i}{\pi} \partial_{\bar{x}}(\phi) \delta^{\alpha \beta}, \\
& j_{-}^{\alpha \beta}(z)=\vec{\psi}_{L}^{\alpha}(z) \psi_{R}^{\beta}(z)-\bar{\psi}_{L}^{\alpha} \psi_{R}^{\beta}-\frac{i}{\pi} \partial_{x}(\phi) \delta^{\alpha \beta}
\end{aligned}
$$

which belong to the first class, and chiral charge changing operators given by

$$
\begin{aligned}
& s_{+}^{\alpha \beta}(z)=\bar{\psi}_{R}^{\alpha}(z) \psi_{R}^{\beta}(z) \rightarrow \bar{\psi}_{R}^{\alpha} \psi_{R}^{\beta} \exp (2 \phi), \\
& s_{-}^{\alpha \beta}(z)=\bar{\psi}_{L}^{\alpha}(z) \psi_{L}^{\beta}(z) \rightarrow \bar{\psi}_{L}^{\alpha} \psi_{L}^{\beta} \exp (-2 \phi)
\end{aligned}
$$

which belong to the second class. The expressions following the arrow signs are in terms of fermion fields redefined according to eq.(2.4). The resulting $\phi$ dependence in eq.(3.2) follows directly from eq.(2.4). On the other hand, the derivation of the $\phi$ dependence in eq.(3.1) is more subtle; one has to either use the anomaly equation eq.(2.5a), or go through a point-splitting procedure in defining the currents.

Our goal is to deduce the spectrum of the theory from the correlations functions. and also to write these functions in factorized form in terms of vertex operators. Along the way, we will verify several analogues of the standard results of instanton physics in four dimensions; among them that chiral symmetry is spontaneously broken without the appearance of a (massless) goldstone mode.

The starting point for the calculation of correlation functions is the standard functional 
integral:

$$
\left\langle j\left(z_{1}\right) j\left(z_{2}\right) \cdots j\left(z_{n}\right)\right\rangle=Z^{-1} \int D \psi D \bar{\psi} D \odot j\left(z_{1}\right) j\left(z_{2}\right) \cdots j\left(z_{n}\right) \sum_{N=-\infty}^{\infty} \exp \left[\int d^{2} z_{\mathcal{L}_{N}(z)}\right] .
$$

In this expression, each $j$ stands for either type of operator given in eq.(3.1) and eq.(3.2), and also, in order to simplify writing, flavor indices are suppressed.

The partition function. $Z$. is given by 2 similar integral without the operator insertions. and $\mathcal{L}_{N}$ is the lagrangian of eq.(2.9). The final sum is over background fields $A_{\mu}^{c}\left(N^{\prime}\right)$, labeled by the monopole charge.$V$. The functional integral over the fermi fields can be carried out by first expanding these fields in eigenmodes of the Dirac operator;

$$
\begin{aligned}
& \dot{H}(z)=\sum_{m=0}^{N-1} c_{m} \eta_{m}(z)+\cdots, \\
& \bar{\Psi}(z)=\sum_{m=0}^{N-1} \bar{c}_{m} \eta_{m}^{*}(z)+\cdots
\end{aligned}
$$

and then integrating over the expansion coefficients $c$ and $\bar{c}$. In the above expression. we displayad only that part of the expansion that suns over the zero modes, since we shall only use the zero modes in the following discussion. The importance of the zero modes is due to the fact that upon substituting the mode expansion eq.(3.4) into $\mathcal{C}_{N}$ we find that the action is independent of $\mathrm{c}$ and $\overline{\mathbf{c}}$ and, therefore (by Brezin integration), the functional integral tanishes, unless each coefficient appears once and only once in the expansion of the bilinear operators. From this observation, the following selection rules easily follow:

a) The partition function $Z$ receives contributions from the $N=0$ sector only.

b) When all the bilinears are in the first class (eq.(3.1)), again only the $N=0$ sector contributes. Since, in this sector, $A_{\mu}^{c}$ can be set equal to zero, the model reduces to a sum of massless free fermions and a single massless free boson. ${ }^{15)}$ Clearly, nothini new or interesting emerges from probing this sector. 
c) Consider correlation functions of only second class bilinears. For the sector with monopole number $N$, a nonvarishing correlation function must contain a mininum number of bilinears in order not to vanish. This occurs when all the zero mode functional integrals art each accompanied by a $\psi$ or $\psi^{*}$ from the corresponding bilinear. The following selection rules are then easy to verify: For $N>0$, the minimal nonvanishing correlation function must be built out of $s_{+}$'s only, and for $N<0$, out of $s_{-}$'s only. Furthermore, referring to eq.(3.2), each flavor index $\alpha$, as well as each index $\beta$, must appear precisely $|\boldsymbol{N}|$ number of times, and the selection rule of eq.(2.12) will be sasisfied. In summary, for a given $N$, the "minimal" correlation functions contains $|N| \kappa$ bilinears of second class, with each fiavor index appearing $|N|$ number of times. Also, the insertions are necessarily all right handed ( $s_{+}$'s) for $N>0$, and only left handed (s-'s) for $N<0$.

We shall now carry out the fermionic integrations for the minimal (simplest nonsanishing) correlation functions. It is clear that the answer will be an antisymmetrized product of the zero mode wave functions, given by eqs.(2.17), and so, up to an overall sign, the particular order in which each flavor index is attached to a particular bilinear is of no consequence. In other words, there is only one rninimal correlation function to compute, and the computation can be done by choosing a particular set of flavor indices that satisfy the selection rules. Furthermore, the result factorizes, so it is sufficient to compute the zero mode contribution for a single flavor and fermion number; the final result is then a product of the partial results for different flavor species and fermion numbers. This is due to the fact that complete factorization is spoiled only by the necessity of skew-symmetry of the wave function for identical fermions; for non-identical fermions, there is no such constraint. At the cost of a slight abuse of notation, let us denote the result for a single species of fermions 
by

$$
\begin{aligned}
& V_{R}^{\alpha} \equiv\left\{\prod_{n=0}^{N-1} \psi_{R}^{\alpha}\left(z_{n}^{\alpha}\right),\right\rangle \\
& \bar{V}_{R}^{\alpha} \equiv\left\{\prod_{n=0}^{N-1} \Psi_{R}^{\alpha}\left(z_{n}^{a}\right)\right\rangle,
\end{aligned}
$$

so that the complete result for positive $N$ is given by

$$
V_{R}=\prod_{\alpha=1}^{\infty} v_{R}^{a} \prod_{\beta=1}^{n} \nabla_{R}^{\beta}
$$

A similar result with $V_{R}$ 's replaced by $V_{L}$ 's holds for negative $N$. For the sake of clarity we have denoted by $z_{n}^{\circ}$ the co-ordinate of the nth insertion of the $\alpha$ flavored bilinear. The expression for the I " s is easily calculated and yields

$$
\eta_{R}^{\circ}=D^{\alpha} \prod_{n=0}^{N-l} h\left(z_{n}^{\alpha}, \bar{z}_{n}^{\alpha}\right)
$$

where $D^{\circ}$ is the Van der Monde determinant:

$$
D^{\alpha}=\operatorname{det}\left|\left(z_{n}^{\alpha}\right)^{m}\right|=\prod_{n>m}\left(z_{n}^{\alpha}-z_{m}^{\alpha}\right),
$$

with $n$ and $m$ taking values from 0 to $N-1$. The result for the $\bar{V}$ 's one cbitains by replacing E's by I's, with the following overall result:

$$
V_{R}=H F \bar{F},
$$

where.

$$
H=\prod_{\alpha=3}^{n} \prod_{m=0}^{N=1} h^{2}\left(z_{m}^{\alpha}, \bar{z}_{m}^{\alpha}\right)=\exp \left\{2 \sum_{\alpha=1}^{n} \sum_{m=0}^{N-1} g\left(z_{m}^{\alpha}, \overline{z_{m}^{\alpha}}\right)\right\},
$$

and

$$
\begin{aligned}
& F=\prod_{a=1}^{n} \prod_{m>n}\left(z_{m}^{\alpha}-z_{n}^{\alpha}\right), \\
& F=\prod_{a=1}^{\infty} \prod_{m>n}\left(\bar{z}_{m}^{\alpha}-\bar{z}_{n}^{\alpha}\right) .
\end{aligned}
$$


Having completed the fermionic part of the functional integration, we now do the integration over the field $\phi$. The lagrangian for $\phi$ is given by eq.(2.9), supplemented by the exponentials in $\phi$ resulting from the redefinition of the fermion fields. (See eq.(3.2)). The resulting functional integral is;

$$
B=\int D \phi \exp \left\{\int d^{2} z \mathcal{L}_{\phi}(z)\right\},
$$

with,

$$
\mathcal{L}_{\phi}=-\frac{\kappa}{2 \pi}\left(\partial_{\mu} \phi\right)^{2}-\frac{\kappa}{\pi} \phi F^{c}+2 \phi(z) \sum_{\alpha=1}^{\kappa} \sum_{m=0}^{N-1} \delta^{2}\left(z-z_{m}^{\circ}\right) .
$$

This gaussian integral is easily done by shifting $\phi$ by

$$
\phi \rightarrow \phi+\phi^{c}
$$

where $\phi$ satisfies

$$
\square \phi^{c}-F^{c}+\frac{2 \pi}{\kappa} \sum_{a=1}^{\kappa} \sum_{m=0}^{N-1} \delta^{2}\left(z-z_{m}^{\alpha}\right)=0
$$

Noting that

$$
F^{c}=-\square g
$$

from eqs. (2.10) and (2.14a), the solution to eq.(3.9b) is

$$
\phi^{e}=-g-\frac{1}{2 \kappa} \sum_{\alpha, n} \log \left|z-z_{n}^{a}\right|^{2} .
$$

It is easy to verify that $\phi^{c}$ is not singular at $|z|=\infty$, since the singularity of $g$ given by eq.(2.14b) is cancelled by the sum over the logarithms. This is as it should be, since the coefficient of the logarithm at infinity measuses the monopole flux, and in the separation of the gauge field given by eq.(2.8), $\phi$ was stipulated not to carry any mcnopole flux. Substituting eq. (3.11) back into eq.(3.8a), we have

$$
B=\Lambda H^{-1} \exp \left\{-\frac{1}{2 \kappa} \sum_{\alpha_{1}, \mathcal{\rho}, m, n} \log \left|z_{m}^{\alpha}-z_{n}^{\rho}\right|^{2}\right\},
$$


where

$$
\Lambda=\exp \left(-\frac{\kappa}{2 \pi} \int d^{2} z g(\square g)\right)
$$

is a normalization constant. The final answer for the minimal correlation function is then the product of three factors: $V_{R}$ given by eq.(3.7), the factor $\Lambda$ given by eq. (3.11b), and a remaining fermionic determinant due to non-zero modes;

$$
\begin{aligned}
\left\langle\prod_{a, 3} s_{+}^{\alpha \beta}\right\rangle & =\Lambda^{\prime} \exp \left\{\sum_{a} \sum_{m>n} \log \left|z_{m}^{\alpha}-z_{n}^{a}\right|^{2}\right. \\
& \left.-\frac{1}{2 \alpha} \sum_{\alpha, \hat{B}, m, n} \log \left|z_{m}^{\alpha}-z_{n}^{\beta}\right|^{2}\right\},
\end{aligned}
$$

with $\Lambda^{\prime}$ given by

$$
\Lambda^{\prime}=\Lambda \operatorname{det}_{0}\left(i \not \partial+A^{c}\right)
$$

The subscript 0 is a reminder that zero modes are to be omitted in computing the determinant. Although we assumed $N>0$ in calculating this result, an identical expression is obtained if we start with $X<0$ and replace $s_{+}$'s by $s^{\prime} . s$.

A few comments about eq.(3.12) are in order. Firstly, the factors involving position variables ( $z$ 's) are completely independent of the details $A_{\mu}^{c}$, or equivalently, of $g$. They: depend only on the monopole charge $N$, and even then only indirectly thorough the range of the indices $m$ and $n$. (Remember, they run from 0 to $N-1$ ). The factor $H$, which depends on $g$. has disappeared completely from the final expression. The normalization constant $A^{\prime}$ could still depend on $g$; it is, however, quite easy to show that it dons not. One makes an infinitesimal change in $A_{\mu}^{e}$ and a compensating change in $\phi$, and using the anomaly equation eq.(2.5a), it is possible to show that these changes cancel. This is a statement of the fact that the result eq.(3.12) is gauge invariant. Thus, the correlation function is. as it should be, independent of the arbitrary split introduced in eq.(2.8). Although the preceding discussion establishes that $\Lambda^{\prime}$ is independent of the details of $A_{u}^{c}$, it may depend on the monopole charge $N$. In the appendix, by evaluating the fermion determinant, we 
shall show that this dependence is of the form

$$
\Lambda_{N}^{\prime}=(\lambda)^{N}
$$

where $\lambda$ is a constant, which can be thought of as an effective coupling constant of the chirality violating process.

Our next task is to deduce the spectrum of the $N \neq 0$ sectors from eq.(3.12). Since eq.(3.12) is in the standard Koba-Nielsen form, it can ive factorized in terms of the wellknown string vertex operators that depend only on the momenta entering the vertices. The exponent in eq.(3.12a) corresponds to the coulomb energy of various charges located at points $z_{m}^{\alpha}$. The momentum flowing into the vertex at $z_{m}^{\circ}$ is simply the charge located there. Comparing ea. $(3.12 a)$ with the expression

$$
W=\frac{1}{2} \sum_{\alpha, \beta, m, n} \mathbf{p}_{m}^{\alpha} \cdot \mathbf{p}_{n}^{\rho} \log \left|z_{m}^{\alpha}-z_{n}^{\rho}\right|^{2}
$$

for the coulomb energy, we arrive at the following condition:

$$
p_{m}^{\alpha} \cdot p_{n}^{\beta}=\delta_{\alpha \beta}-\frac{1}{\kappa}
$$

The minimal solution to these equations can be expressed in terms of $\kappa$ orthonormal vectors $\hat{p}^{\mathbf{a}}$ as

$$
\mathbf{p}_{m}^{\alpha}=\tilde{p}^{\alpha}-\frac{1}{\kappa} \sum_{\beta} \hat{p}^{\beta}
$$

where

$$
\hat{p}^{a} \cdot \hat{p}^{\beta}=\delta_{\alpha \beta}
$$

It is important to notice that the momenta do not depend on the subscript $m$, and therefore, the solution eq. (3.15a) is valid for all $N$. This means that we can forget about the monopole flux and simple assign moment2 to both fermions and antifermions in accordance with their flavor only through eq.(3.15a). The constraint

$$
\sum_{a=1}^{n} p_{m}^{a}=0 \text {, }
$$


satisfied for all $m$, guarantees overall momentum conservation

$$
\sum_{\alpha_{1} m} p_{m}^{a}=0
$$

if and only if each flavor index occurs with the same multiplicity. This multiplicity is exactly $N$, the monopole charge. An identical momentum assignment can be made for left handed fermions when $N$ is negative.

It is of interest to investigate the mass spectrum that follows from the momentum. assignment eq.(3.15). Consider a thanıel in which the ath flavor occurs $n^{\alpha}$ number of times, where $n^{\alpha}$ is a non-negative integer. The lowest squared "mass" in this channel is

$$
\begin{aligned}
M^{2} & =\left(\sum_{\alpha} n^{\alpha}\left(p^{\alpha}-\frac{1}{\kappa} \sum_{\beta} \rho^{\alpha}\right)\right)^{2} \\
& =\sum_{\alpha}\left(n^{\alpha}\right)^{2}-\frac{1}{\kappa}\left(\sum_{\alpha} n^{\alpha}\right)^{2} .
\end{aligned}
$$

Of course, starting with the lowest $M^{2}$, there are integerly spaced higher excited states. If it were not for the second term in eq.(3.18), this spectrum could be identified with the spectrum resulting from toroidal compactification in $\approx$ dimension. Eq. (3.18) corresponds to a compactification on a Lorentz lattice with a constraint and although the lattice has an indefinite metric the spectrum is readily seen to be non- negative (t.tis is ammended by including contributions to $M^{2}$ from string excitations).

Let us now examine corselation functions with a non-minimal number of second class bilinears for a given $N$, which, for the sake of definitenes, is again taken to be positive. In this case, there are a certain number of left-handed bilinears ( $s_{-}$'s), in addition to $s_{+}$'s. The extra $\psi_{L}$ 's will then contract with some of the $\bar{\psi}_{R}$ 's, and the extra $\bar{\psi}_{L}$ 's will contract with $\psi_{R}$ 's, leaving behind an equal number (i.e. $N$ ) of uncontracted $\psi_{R}$ 's and $\bar{\psi}_{R}$ 's. The uncontracted fermions make up the "minimal" correlation function which absorbs all the 2ero modes, and which has already been evaluated. The contractions will result in a product 
of two point functions, to be calculated in the presence of the external field $A_{\mu}^{c}$ given by eq. (2.14a). The two point functions, defined by,

$$
\begin{aligned}
& \left\langle\psi_{R}^{a}(z) \bar{\psi}_{L}^{\beta}\left(z^{\prime}\right)\right\rangle=\delta_{\alpha \beta} G_{12}\left(z, z^{\prime}\right), \\
& \left\langle\psi_{L}^{Q}(z) \bar{\psi}_{R}^{S}\left(z^{\prime}\right)\right\rangle=\delta_{\alpha \beta} G_{21}\left(z, z^{\prime}\right),
\end{aligned}
$$

satisfy the following equations:

$$
\begin{aligned}
& 2 i\left(\partial_{\bar{z}} G_{12}-\partial_{\bar{z}}(g) G_{12}\right)=\delta^{2}\left(z-z^{\prime}\right), \\
& 2 i\left(\partial_{z} G_{11}+\partial_{z}(g) G_{21}\right)=\delta^{2}\left(z-z^{\prime}\right)-\sum_{m=0}^{N-1} \eta_{m}(z) \eta_{m}^{*}\left(z^{\prime}\right)
\end{aligned}
$$

The sum that appears on the right hand side of the second equation is over the positive chirality (right handed) zero modes defined by eq.(2.17a). In computing the $\bar{G}$ 's, the functional integral over $\psi_{R}$ and $\bar{\psi}_{R}$ is performed over only the non-zero modes, since the zero modes have already been accounted for by the minimal part of the correlation function. The delta function on the right hand side of the second equation in eq.(3.20) has then to be projected into the orthogonal complement of the space spanned by the zero modes, thereby: explaining the appearance of the summation term. Of course, eq.(3.20) are only valid for $N>0$; for $N<0$, the roles of left and right, i.e. $G_{12}$ and $G_{31}$ have to be interchanged.

The solution to eq. $(3.20)$ is easy to write down:

$$
\begin{aligned}
& G_{12}\left(z, z^{\prime}\right)=\frac{1}{2 \pi i} \frac{h(z) h^{-1}\left(z^{\prime}\right)}{z-z^{\prime}}-\sum_{m=0}^{N-1} \eta_{m}(z) x_{m}^{*}\left(z^{\prime}\right), \\
& G_{21}\left(z, z^{\prime}\right)=\frac{1}{2 \pi i} \frac{h^{-1}(z) h\left(z^{\prime}\right)}{\bar{z}-z^{\prime}}-\sum_{m=0}^{N-1} \chi_{m}(z) \eta_{m}^{*}\left(z^{\prime}\right),
\end{aligned}
$$

where $\chi_{m}$ are solutions to the equation

$$
2 i\left(\partial_{z} \chi_{m}+\partial_{s}(g) \chi_{m}\right)=\eta_{m}
$$

After having gone through the trouble of projecting out the zero modes from the two point functions, we shall now argue that such a projection is unnecessary. In computing 
the contractions, one can replace eq.(3.21) by the following simplified version:

$$
\begin{aligned}
& G_{12}-\tilde{G}_{12}=\frac{1}{2 \pi i} \frac{h(z) h^{-1}\left(z^{\prime}\right)}{z-z^{\prime}}, \\
& G_{21}-\tilde{G}_{21}=\frac{1}{2 \pi i} \frac{h^{-1}(z) h\left(z^{\prime}\right)}{\bar{z}-\bar{z}^{\prime}} .
\end{aligned}
$$

To see how this comes about, note that the extra terms in sum in eq.(3.21) contain a zero mode wave function $\eta_{* n}$ or $\eta_{\mathrm{m}}^{*}$. By fermi statistics, the product of these wave functions have to be antisymmetrized. so that for a fixed flavor index, each value of $m$ can occur at most once. But each value of $m$ has already appeared once in the "minimal" part of the correlation function; hence, the contribution of this extre term vanishes.

Having taken care of the fermionic integration, we now return to the functional integration in the of field. Immediately one sees that in this case, eq.(3.8b) is amended by additional terms of both signs. This is due to the fact that we have additional $s_{-}$'s and $s_{+}$'s and with the $s_{-}$'s there appear negative exponents of the field $\phi$ compared to $s_{+}$'s. (see eq.(3.2)). Equation (3.8b) is therefore replaced by

$$
\mathcal{L}_{\phi}=-\frac{\kappa}{2 \pi}\left(\partial_{\mu} \phi\right)^{2}-\frac{\kappa}{\pi} \phi F^{\epsilon}+2 \phi(z) \sum_{\alpha, m} q_{m}^{\alpha} \delta^{2}\left(z-z_{m}^{a}\right),
$$

where $q_{m}^{o}$ is +1 or -1 , depending on whether the insertion at $z_{m}^{a}$ is an $s_{+}$or an s- respectively. The integration over $\phi$ can be carried out as before, with the result that the flux dependent factors $h$ and $h^{-1}$ in eq.(3.23) are again canceled, just as they did in deriving eq.(3.12a). We will not exhibit the final answer, since it is somewhat complicated to write down. It turns out, however, that the result can be compactly written as a factorized operator expression in terms of free fermion and boson fields. The appearance of free fermions is not surprising since aiter the cancellation of the factors $h$ and $h^{-1}$ in eq.(3.23) one is left with free fermion propagators. Let us then define massless free fermion $f$ Ids $\psi_{O, R}^{\circ}$ and $\psi a, L$, with the two point functions

$$
\left(\psi_{O, L}^{\alpha}(z) \bar{\psi}_{C, L}^{\beta}\left(z^{\prime}\right)\right)=\frac{1}{2 \pi i} \frac{1}{z-z^{\prime}} \delta_{\alpha \beta},
$$




$$
\left\langle\psi^{\prime} \circ, L(z) \bar{\psi}_{O, R}^{G}\left(z^{\prime}\right)\right\rangle=\frac{1}{2 \pi i} \frac{1}{\bar{z}-\bar{z}^{\prime}} \delta_{\alpha \beta} .
$$

Since the field $\phi$ of eq.(3.24) is essentially a free boson field, it is convenient to define two fields, cue right moving and one left moving, by

$$
\begin{aligned}
\left\langle\phi_{+}(z) \psi_{+}\left(z^{\prime}\right)\right\rangle & =-\log \left(z-z^{\prime}\right), \\
\left\langle\phi_{-}(z) \psi_{-}\left(z^{\prime}\right)\right\rangle & =-\log \left(\bar{z}-\bar{z}^{\prime}\right) \\
\left(\phi_{+} \psi_{-}\right\rangle & =0 .
\end{aligned}
$$

Finally, to take care of the contribution of the zero modes, wa define four sets of scalar fields, labeled by the flavor index $a$ :

$$
\begin{aligned}
& \left\langle\phi_{+, R}^{\alpha}(z) \phi_{+, R}^{\beta}\left(z^{\prime}\right)\right\rangle=-\delta_{\alpha \beta} \log \left(z-z^{\prime}\right), \\
& \left\langle\phi_{+, L}^{\alpha}(z) \phi_{+, L}^{\beta}\left(z^{\prime}\right)\right\rangle=-\delta_{\alpha \beta} \log \left(z-z^{\prime}\right), \\
& \left\langle\phi_{-, R}^{\alpha}(z) \phi_{-, R}^{\beta}\left(z^{\prime}\right)\right\rangle=-\delta_{\alpha \rho} \log \left(\bar{z}-\vec{z}^{\prime}\right), \\
& \left\langle\phi_{-, L}^{\alpha}(z) \phi_{-. L}^{\beta}\left(z^{\prime}\right)\right\rangle=-\delta_{\alpha \beta} \log \left(\bar{z}-\bar{z}^{\prime}\right) .
\end{aligned}
$$

Two point functions with mixed indices are, as usual, defined to be zero. The fermionic bilinears can now be expressed in terms of these free fields as follows:

$$
\begin{array}{r}
\bar{\psi}_{R}^{a}(z) \psi_{R}^{\beta}(z)-\exp \left[(2 \kappa)^{-\frac{1}{2}}\left(\phi_{+}(z)+\phi_{-}(z)\right)\right] \\
\left\{\exp \left[2^{-\frac{1}{2} i}\left[\phi_{-, R}^{\alpha}(z)+\phi_{+, R}^{\rho}(z)\right]\right]+\bar{\psi}_{O, R}^{a}(z) \psi_{O, R}^{\beta}(z)\right\}, \\
\vec{\psi}_{L}^{a}(z) \psi_{L}^{\beta}(z) \rightarrow \exp \left[-(2 \kappa)^{\frac{1}{2}}\left(\phi_{+}(z)+\phi_{-}(z)\right)\right] \\
\left\{\exp \left(2^{-\frac{1}{2}} i\left[\phi_{+, L}^{\alpha}(z)+\phi_{-. L}^{\beta}(z)\right]+\bar{\psi}_{O, L}^{a}(z) \psi_{O, L}^{\beta}(z)\right\} .\right.
\end{array}
$$

To establish this correspondence, one must show that the correlation functions are correctly reproduced by the right hand side of eq.(3.28). The exponential factor in front 
obviously reproduces the result of integrating over $\phi$, and the bilinears in free fermions provide the fermionic contractions. Comparing with the first term on the right hand side of eq.(3.12a), it is also easy to verify that the exponential in flavored scalar fields $\phi^{\circ}$ reproduces the contribution of the zero modes. 


\section{The Torus and Modular Invariance.}

In this section, we study the model on a surface of higher genus, namely, the torus, and pay particular attention to the modular properties of theory. Consider the two dimensional field theory given by eq. $(2.9)$ on a torus corresponding to the parallelogram with side $(0,1)$ and $(c, d)$, where

$$
T=c+i d
$$

is the modular parameter (see Fig. 1). The problem is simplified considerably by choosing the flux density $F^{c}$. defined by eq.(2.10), to be constant over the torus. This choice has the advantage of being modular invariant: it is therefore possible to study the modular properties of the fermion sector separtely, without having to worry about the scalar field $\phi$. Furthermore, the problem of solving the Dirac equation in the presence of a uniform external magnetic field is a standard one going all the way back to Landau, and it has recently been studied by several authors frorn different points of view. ${ }^{21), 18\}, 19)}$ In the interests of completeness and clarity, we present a brief exposition below.

Let us begin by noting that the monopole quantization condition, eq.(2.i) valid for surfaces of arbitrary genus in this case reads

$$
F^{c} d=2 \pi N
$$

where $N$, as before, is the integer monopole number. Without any loss of generality: we can make the following convenient gauge choice for $A_{\mu}^{c}$ (see eq.(2.3a)):

$$
A_{\nu}^{c}=0, A_{x}^{c}=-F^{c} y=-\frac{2 \pi N}{d} y .
$$

We are interested in finding the eigenfunctions of the Dirac operator

$$
i p=i p+A^{e}
$$

on the torus. In this section we solve the massless Dirac equation and investigate the modular groups action on these zero modes. In the appendix the massive Dirac equation 
is solved and it is shown that these non-zero modes transform exactly as the zero modes under modular transformations.

The equation for the zero modes, eq.(2.13) with potential given by eq.(4.3), has the following general solutions:

$$
\begin{aligned}
& \eta_{R}=f_{R}(z) \exp \left(-\frac{\pi N y^{2}}{d}\right), \\
& \eta_{L}=f_{L}(\bar{z}) \exp \left(\frac{-\pi N y^{2}}{d}\right),
\end{aligned}
$$

where the $f$ 's are analy'tic functions in their respective arguments and are determined by the Ehoice of the boundary conditions. Along the horizontal direction, we impose the standard periodic $(P)$, or antiperiodic $(A)$ boundary conditions

$$
\eta(z+1)= \pm \eta(z)
$$

We will show later that this choice of boundary conditions is the minimal set for which one can construct modular covariant Green's functions. We pause to remark that in the $\Lambda=0$ (fre) case it is the requirement of modular covariance at higher loop ( $g \geq 2$ ) that picks out eq.(4.6) as the minimal set of boundary conditions but with $N \neq 0$ it will be shown that this spin structure is necessary at one loop. As a result of the non-vanishing total flux the torus (eq.(4.21)), we cannot impose a simple boundary condition like eq.(4.6) in the $\bar{\tau}$ direction. This follows from the fact that in an ambient magnetic field the two independent covariant translation operators no longer commute and therefore cannot be simultaneously diagonalized. The most general boundary conditions in the $\dot{\tau}$ direction consistent with non-vanishing total flux, turn out to be

$$
\eta(z+\tau)=\exp (-2 \pi i N x+i \delta) \eta(z)
$$

where $\delta$ is an arbitrary constant ( $z$ independent) phase. These boundary conditions are readily satisfied by taking $f$ 's to be suitable modular functions i.e. Jacobi theta functions ${ }^{22)}$; 
however, both $\eta_{R}$ and $\eta_{L}$ cannot simultaneously be normalizable. If $N>0$, the only normalizable solution for $\eta_{L}$ is trivial:

$$
\eta_{L}=0
$$

whereas, 2 special solution for $\eta_{R}$ is obtained by taking

$$
f_{R}=\theta_{3}(N z, N r)
$$

where $\theta_{3}$ is one of the standard Jacobj theta functions. For $N<0$, the roles of $R$ and $L$ are reversed. Without any ioss of generality we henceforth take $N$ positive.

The solution eq.(4.8b) satisfies the boundary condition eq.(4.7) with

$$
\delta=-\pi N c
$$

To find the general solution corresponding to an arbitrary $\delta$, we take advantage of translation invariance: We can translate the coordinate $z$ in eq. $(4.8 \mathrm{~b})$ by an arbitrary complex quantity to to construct a whole set of new solutions. However, such a translation must be accompanied by a non-trivial phase factor in order to satisfy eq.(4.7):

$$
f \rightarrow \exp \left[\frac{2 \pi i N y_{0} z}{d}\right] \theta_{3}\left(N\left(z+z_{0}\right), N \tau\right)
$$

The boundary condition eq.(4.7) is now satisfied, but the other boundary condition given by eq.(4.6) is violated, unless we require that

$$
\frac{N y_{0}}{d}=\frac{\pi}{2}
$$

where $n$ is an integer. The corresponding zero mode $\eta$ is given by

$$
\eta=\exp \left(-\frac{\pi N y^{2}}{d}+\pi i n z\right) \theta_{3}\left(N z+\frac{n}{2} r+a, N r\right),
$$

where, for convenience, we have defined

$$
a=N x_{0}-\frac{n c}{2} .
$$


We note that even $\pi$ correspond to periodic toundary conditions in the $x$ direction, whereas odd $n$ yield antiperiodic functions. The range of the integer $n$ can be taken to be from 0 to $2 N-1$, and the range of the real number " $a$ " is continuous, there are then infinitely many solutions! However, only a finite number of them are modular covariant, by which we mean that they transform into linear combinations of themselves under modular transformations. It is well known that the modular group can be generated by two special group elements:

$$
z \rightarrow z, \quad \tau \rightarrow \tau+1
$$

and,

$$
z \rightarrow z^{\prime}=-\frac{z}{\tau}, \quad T \rightarrow \tau^{\prime}=-\frac{1}{\tau} .
$$

To test whether $\eta$ given in eq.(4.12a) is covariant under theses mappings, we need some standard identities for theta functions. The identities that are useful with eq. (4.13a) read

$$
\theta_{3}\left(N z+\frac{n}{2} \tau+a, N(\tau+1)\right)=\theta_{3}\left(N z+\frac{n}{2} \tau+a, N \tau\right)
$$

for even $N$ and,

$$
\theta_{3}\left(N z+\frac{n}{2} \tau+a, N(\tau+1)\right)=\theta_{3}\left(N z+\frac{n}{2} \tau+a-\frac{1}{2}, N \tau\right)
$$

for odd $N$.

Hence, for even $N$ there is no constraint and for odd $N$ if a certain value of $a$ appears. then the value $a-\frac{1}{2}$ must also appear. The transformation given by eq. $(4.13 b)$ is more complicated; to implement it, we need the following series of identities:

$$
\theta_{3}\left(N z+\frac{n}{2} \tau+a, N \tau\right)=(-i \tau)^{-\frac{1}{2}} \exp \left(-i \pi \frac{\left(N z+\frac{\pi}{3} \tau+a\right)^{2}}{N \tau}\right) \theta_{3}\left(z^{\prime}+\frac{a}{N} \tau^{\prime}-\frac{n}{2 N}, \frac{\tau}{N}\right)
$$

and,

$$
\theta_{3}\left(z^{\prime}+\frac{a}{N} \tau^{\prime}-\frac{n}{2 N}, \frac{\tau^{\prime}}{N}\right)=\sum_{\ell=0}^{N-1} \exp \left\{\frac{\pi i \ell^{2} \tau^{\prime}}{N}+2 \pi i \ell\left(z^{\prime}+\frac{a}{N} \tau^{\prime}-\frac{n}{2 N}\right)\right\} \theta_{3}\left(N z^{\prime}+(a+\ell) \tau^{\prime}-\frac{n}{2}, N^{\prime} \tau^{\prime}\right)
$$


Comparing the argument of the theta function on the right hand side of eq. $(4.15 \mathrm{~b})$ with that in eq.(4.12a), it is clear that we must require

$$
a+\ell=\frac{n^{\prime}}{2}
$$

where $n^{\prime}$ is an integer. Since $\ell$ is also an integer and $a$ is defined up to an additive integer, its values can be restricted to 0 and $\frac{1}{2}$. We express this by setting

$$
a=\frac{m}{2},
$$

where $m=0$ or 1 . Modular invariance therefore restricts the allowed zero modes to the following discrete set conveniently labeled by two integers $m$ and $n$ :

$$
n_{m, n}(z, \tau)=\exp \left\{\pi i n z-\frac{\pi N y^{2}}{d}+\frac{\pi i n^{2} \tau}{4 N}\right\} \theta_{3}\left(N z+\frac{n \tau}{2}+\frac{m}{2}, N \tau\right),
$$

wbere $m=0,1$ and $n=0.1, \ldots, 2 N-1$. The even (odd) values of $n$ and $m$ correspond to periodic (antiperiodic) solutions in the $\hat{x}$ and $\hat{f}$ directions respectively. We have introduced an additional multiplicative constant on eq.(4.18) compared to eq.(4.12a) in order to simplify its transformation properties under the modular group. In what follows there will be no need to normalize solution eq.(4.18). We also note that the number of zero modes is in agreement with the index theorem eq.(2.18): If we consider only $P P$ (periodic in both directions) solutions, $n=$ even and $m=0$, there are then $N$ zero modes which is the correct number. When anti-periodic solutions are also included, one must consider fourfold covering of the torus, which, while quadrupling the total flux also increases the number of solutions to $4 N$.

The zero mode solutions defined by eq.(4.18) have simple transformation properties under the modular group given by eq.(4.13). Considering first the translation given by (4.13a), we have,

$$
\eta_{n, n}(z, \tau+1)=\exp \left(\frac{\pi i n^{2}}{4 N}\right) \eta_{m+n, n}(z, \tau)
$$


for $N=$ even and,

$$
\eta_{m, n}(z, v+1)=\exp \left(\frac{\pi i n^{2}}{4 N}\right) \eta_{m+n+1, n}(z, \tau),
$$

for $N=$ odd. Using the abbreviation $(P, P),(P, A)$, etc. to denote periodic $(P)$ or antiperiodic $(A)$ boundary conditions along $\hat{x}$ and $\dot{\tau}$ directions, we can express the content of eq.(4.19) in the following way: Under $\tau \rightarrow \tau+1$, we have for even $N$

$$
\begin{aligned}
& (P, P)=(P, P),(A, P)=(A, A) \\
& (P, A)=(P, A)
\end{aligned}
$$

and, for odd $N$

$$
\begin{aligned}
& (P, P)=(P, A),(A, P)=(A, P) \\
& (A, P)=(A, A) .
\end{aligned}
$$

For even $N$, the modular transformation properties of fermions are unchanged by the presence of the monopole background; they are identical to the free fermion transformations. For add $N$, one sees that switching $A$ and $P$ in eq.(4.20b) we reproduce eq.(4.20a). One way to think of this change of statistic is that for odd $N$ the angular mornentum in the gauge field of the fermion-monopole systen will be a half-odd integer so the definition of a fermionic system for even $N$ will be different from that at odd $N$.

Consider now the inversion given by eq. $(4.13 \mathrm{~b})$. We have

$$
\eta_{m, n}(z, \tau)=\exp (i x) \sum_{\ell=0}^{N-1} \exp \left\{-\frac{\pi i}{N} n\left(\ell+\frac{m}{2}\right)\right\} \eta_{m+2 \ell, n}\left(z^{\prime}, \tau^{\prime}\right),
$$

where,

$$
x=-\pi N \operatorname{Re}\left(\frac{z^{2}}{\tau}\right)
$$

The overall phase in this transformation can be understood as follows: the transformation eq. (4.13a) interchanges the two sides of the the basic parallelogram, which results 
in a change from the gauge given in eq. (4.3) to a new gauge given by

$$
A_{\mu} \rightarrow A_{\mu}+\partial_{\mu} \chi
$$

where $x$ is precisely given by eq.(4.21b). We can again express the covariance of these solutions by studying bow the transformation $\tau \rightarrow-1 / \tau$ exchanges solutions of various boundary conditions. Under $z \rightarrow-\frac{x}{\tau}, \tau \rightarrow-\frac{1}{\tau}$ For all $N$,

$$
(P P)=(P, P),(A, A)=(A, A),(A P)=(P, A)
$$

These are again identical to the free field transformations. As a result, just as in the free field case, we obtain a modular invariant answer by summing over the four spin structure $(P, P),(A, P),(P, A)$ and $(A, A)$. Here, however, we have a slightly stronger result: For odd $X,(P, P)$ is not modular invariant by itself (see eq. $(4.20 \mathrm{~b})$ ), so it is really necessary to sum over all of the four spin structures. In contrast, in the free case, $(P, P)$ is modular invariant on the torus, and one has to consider higher genus surfaces to obtain the above result.

One final point concerns the GSO projection. We know that the summation over the spin structures is equivalent to projecting out the sector of the spectrum with odd fermion number. In the free field case, one can carry out this projection in the even and odd chirality sectors separately, since the left and right fermion numbers are separately conserved. In the presence of the monopole background, however, only the total fermion number is conserved. and therefore only a single GSO projection is allowed.

In closing this section, let us summarize what we have learned so far. We have shown that zero mode solutions on the torus trarsform into linear combinations of themselves; this is certainiy necessary for modular invariance, but is it sufficient? The simplest criteria is the invariance of the fermionic determinant on the torus; but in the presence of the non-trivial monopole background, this determinant vanishes because of the existence of the zero modes, and so it is trivially invariant. One would then compute chirality changing 
correlation functions on the torus and examine their modular properties. We have not carried out such a calculation; however, we wish to point out a feature which can deduced with any detailed calculations. Modular transformations are accompanied by chiral (axial) rotations of the world sheet fermions; this is true even for free fields and remains true in the presence of the monopole background (see appendix). In the case of free fields, however, chirality is conserved, and the phases due to the chiral rotations cancel. On the other hand, in the presence of the monopole background, we have chirality changing correlation functions, which will then acquire a phase under modular transformations. We do not think that this destroys the consistency of string theories based on the model presented here; however, this point needs to be investigated further. 


\section{Summary and Poarible Extennions}

In this paper, we have presented a conformal field theory, based on a topological extension of a class of standard coset construction. The model has possible applications to string theory and two dimensional statistical mechanics, as well as being of interest in its own right. It is also an example of an instanton calculation that can be carried out exactly.

The coset construction we considered was based on gauging the $U(1)$ subgroup. It is of some interest to generalize this to models where a non-abelian group is gauged. Such models are quite complicated and usually end up being equivalent to a W.Z.W. model. Without going into the details, we can try to guess what effect monopoles will have on the general features of these models. In the non-abelian case, the functional integral over the gauge field on a compact two dimensional surface can be classified by its topology. For example, consider the surface of a sphere $\left(S_{2}\right)$, the inequivalent gauge fields are classified according to the center of the non-abelian group, which means that there is typically a multiplicative conserved quantim number $Z_{n}$ which replaces the additive monopole charge in the abelian case. We expect that most of the results we have obtained will remain true with the replacement of $Z$ (the set of monopole charges in $U(1)$ monopole) by $Z_{n}$. For example, in the non-abelian case chirality will be broken modulo $n$, etc. This may be a promising line of investigation for future work. 


\section{Acknowledgments}

We wish to thank D. Altschuler, O. Alvarez, J. Freericks, M.B. Halpern, M. Porati, H. Sonoda and E. Tomboulis for interesting comments and discussions relating to this work. This work was supported in part by the Director, Office of Energy Research, Office of High Energy and Nuclear Physics. Division of High Energy Physics of the U.S. Department of Energy under Contract DE-AC03-i6SF00098 and :a part by the National Science Foundation under grant PHY85-1585i. 


\section{Appendix}

In this appendix we solve the eigenvalue equation

$$
\text { i } p_{A} \Psi=\lambda \Psi \quad \Psi=\left(\begin{array}{l}
\psi_{R} \\
\psi_{L}
\end{array}\right)
$$

on the torus with "Ceichmüller parameter $r=c+i d$ where $A_{\mu}$ is as given in eq. (4.3). Squaring $p_{A}$ to diagonalize the above eigenvalue equation we learm

$$
\lambda^{2}=\frac{2 \pi}{d}[|N|(2 \ell+1) \pm N] \begin{cases}- & \Psi_{R} \\ + & \Psi_{L}\end{cases}
$$

where $\ell$ is a non-negative integer corresponding to the mode of the harmonic oscillator (to which $D_{A}{ }^{2}$ reduces), often in these types of problems called the Landau level. As discussed earlier, for $\ell=0$ either $\psi_{R}(N>0)$ or $\psi_{c}(N<0)$ will have a nontrivial zero mode solution. For arbitrary $\ell$ the solution to eq.(A.1) is (for definiteness taking $N>0$ );

$$
\Psi_{\ell .(j)}=\left(\begin{array}{c}
\psi_{(j)}^{\ell} \\
f \psi_{(j)}^{\ell-1}
\end{array}\right)
$$

where $(j)$ indexes any possible degeneracy at level $\ell$ an $! f$ is a constant that depends on $\ell .(j)$ and the normalization convention. Adopting the same boundary conditions as outlined in section 4 , we find that, as expected, each level is exactly $4 N$-fold degenerate, and the most general wave function for each component of the spinor in eq.(A.3) at level $\mathcal{E}$ is a superposition of basis functions labeled by $m$ and $n$ :

$$
\begin{aligned}
\psi_{(m, n)}^{\ell} & =\sum_{k} \exp \left\{-\frac{\pi N y^{2}}{d}+i \pi n z+i \frac{\pi n^{2} \tau}{N}+i \pi k^{2} N \tau\right. \\
& \left.+2 \pi i k\left(N z+\frac{n \pi}{2}+\frac{m}{2}\right)\right\} H_{\ell}\left[\left(\frac{2 \pi N}{d}\right)^{\frac{3}{2}}\left(y+\frac{k N+\frac{n}{2}}{N} d\right)\right],
\end{aligned}
$$

where $n$ is an integer between 0 and $2 N-1$ and $m$ is eitber 0 or 1 . The spin sector identification is the same as before. (See the paragraph following eq.(4.18)). $H_{\ell}$ denotes the $e^{\text {th }}$ Hermite polynomial. In this form one sees immediately that under the modular 
transformation $\tau-\tau+1$ the solutions eq.(A.4), for every $C$, transform precisely the same way as the zero modes, as is shown in eqs. $(4.20 \mathrm{a}, \mathrm{b})$.

To consider the action of solutions eq.(A.4) under $\tau \rightarrow-1 / \tau, z \rightarrow-z / \tau$ it is con:ieritent to use the generating function for the Hermite polynomiais;

$$
e^{-x^{2}+2 s y}=\sum_{\ell=0}^{\infty} \frac{s^{l}}{\ell !} H_{l}(y)
$$

to cast a sum in $\ell$ (the Landau level) of the solutions eq.(A.4) into ( $m, n$ fixed);

$$
\begin{aligned}
R(s, n, m) & =\sum_{i=0}^{\infty} \frac{s^{\ell}}{\ell !} \psi^{\ell}(n, m) \\
& =\exp \left(\frac{-\pi x^{2}}{d}+i \pi n z-s^{2}+2 s \sqrt{\frac{2 \pi N}{d}}\left(y+\frac{n d}{2 N}\right)\right) \\
& \times \theta_{3}\left(N z+\frac{n \tau}{2}+\frac{m}{2}-i s \sqrt{\frac{2 N d}{\pi}}, N \tau\right)
\end{aligned}
$$

Proceeding with the modular transformation $\tau \rightarrow-1 / \tau$ in the fashion outlined in section 4 and using the identities eq. $(4.15 \mathrm{a})$ and eq.(4.15b), we find that a solutions with definite $n, m$ again becomes a linear combination of the solutions in the other sector (identical in form to $4.21 \mathrm{a}$ ) and that the generating function parameter s scales by the factor $e^{i \phi}=\sqrt{\frac{\tau}{7}}$. Expanding the generating function we then find that the $\ell^{\text {th }}$ solution acquires an overall phase $e^{i l s}$ so that the general solution eq.(A.3) transforms as

$$
\Psi_{\ell}=\left(\begin{array}{c}
\psi^{\ell} \\
f \psi^{\ell-1}
\end{array}\right) \rightarrow\left(\begin{array}{c}
e^{i \phi} \psi^{\ell} \\
f \psi^{\ell-1}
\end{array}\right) \times \text { phase. }
$$

which is, of course, precisely what one would expect from the behavior of the two-dimensional Dirac equation (eq.(A.1) or the free dirac equation) under modular transformations.

Finally, from the eigenvalues given by eq.(A.2), the determinant of the non-zero modes can be computed using a suitable regularization, for example, the zeta function regularjzation. It is then easy to show that the dependence of the determinant on $N$ is trivial, justifying eq.(3.13). 


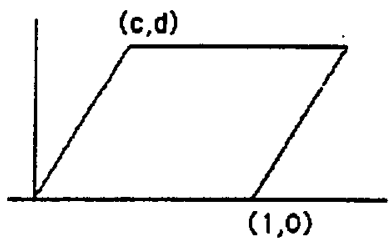

Figure 1 


\section{Bibliography}

1. J. Sherk and J. Schwarz, Phys. Lett. 82b, 60 (1979).

2. K.S. Narian, Phys. Lett. 168B, 41 (1986).

3. P. Candelas, G. Horowitz, A. Strominger and E. Witten, Nucl. Phys. B258, 46 (1985).

4. L. Dixon. J. Hariey; C. Vafa and E. Witten, Nucl. Phys. B261, 678 (1985); Nucl. Phys. B274, 285 (1986).

5. D. Gepner and E. Witten. Nucl. Phys. B278, 493 (1986).

6. K.S. Narian, M. H. Sarmadi and E. Witten, Nucl. Phys. B270, 369 (1987).

7. K.S. Narian, M.H. Sarmadi and C. Vafa, Nucl. Phys. B288, 551 (1987).

8. I. Antoniadis, C. P. Bachas and C. Kounnas, Nucl. Phys. B289, 87 (1987).

9. K. Bardalici and MI. Halpern, Phys. Rev. D3, 2493 (1971).

10. A.N. Redlich, H. J. Schnitzer and K. Tsokos, Nucl. Phys. B289, 397 (198i).

11. K. Bardakci. E. Rabinovici and B. Säring, Nucl. Phys. B299, 151 (1988)

12. A.A. Belavin, A.N. Polyakov and A.B. Zamolochikov, Nucl. Phys. B241, 333 (1984).

13. V.G. Krizhnik and A.B. Zamolochikov, Nucl. Phys. B247, 83 (1984).

14. P. Goddard, A. Kent and D. Olive, Phys. Lett. 152B, 88 (1985); Comm. Math. Phys. 103, 105 (1986).

15. E.T. Tomboulis, Phys. Lett. 198B, 165 (198T).

16. H.J. Schnitzer and K. Tsokos, Nucl. Phys. B291, 429 (1987). 
17. J. Bagger, D. Nemeschansky, N. Seiberg and S. Yankielowicz, Nucl. Phys. B280, 53 (1987).

18. A.Abouelsaood, C.G. Callan, C.R. Nappi and S.A. Yost, Nucl. Phys. B289, 599 (1987).

19. S. Blau, M. Visser and Andreas Wipf, Int. J. Mod. Phys. A4, 1467 (1989)

20. S. Coleman "The International School of Subnuclear Physics" (1981) pg.21.

21. F.D.M. Faldane and E.H. Rezayi, Phys. Rev. B31 No.4, 2529 (1985).

22. D. Mumford "Tata Lectures on Theta" Vol.I Progress in Mathematics No. 28 Birkhäuser (Boston, 1983). 


\section{Contributions and Interests}

This author was deeply involved in this work from its inception. The motivation for the work was my advisor's, $K$. Bardakci, and my contributions to the work include understanding the quantization condition on arbitrary Rieman surface (eq.(2.7)), understanding the consequences of the index theorm (eq.(2.12)) in the plane for singular distributions of flux (which lead to the selection rules of eq. $(2.17 \mathrm{a}, \mathrm{b})$ and underestanding how to combine the eq. $(2.1 \mathrm{ia}, \mathrm{b})$ in the path integral of eq. (3.3) in such a way as to show that the final result (eq. $(3.7 a, b, c)$ and eq, $(3.11 \mathrm{a}, \mathrm{b})$ to give eq. $(3.12 \mathrm{a}, \mathrm{b}))$ is indeed gauge invariant and independant of the distribution of flux (see eq.(3.8) through eq.(3.11)) Also much energy and time was expended checking the results of the next section in which the correlators are rewritten in terms of free field correlators.

Section IV which deals with the issue of including monopole backgrounds on the world sheet of the torus. This author contributed to solving for the zero modes (eq. (4.5) and eq. (4.8b) leading to eq. (4.18). understanding what boundary conditions one should take for them and finally showing modular covariance of the zero modes (eq.(4.19) and eq.(4.21)

resulting in eq.(4.20) and eq.(4.23) respectively). This author was also involved in showing that the modular properties of the massive modes are the same as those for the massless modes (the appendix). furthermore this author was able to make the connection between the change in the modular properties and the statistics of the modes as spacetime fields to make the modular properties found easily understandabie.

In addition to computing and checking results This author was also involved in the writing of various sections and checking drafts and publication proofs.

This author found the following points to be of particular interest in this work. First. the computation of the correlators and the notion of using nontrivial backgrounds to 
undersiand properties of the theory's cluster decomposition and topolological expansion. These ideas have motivated this author to study other systems (such as Chern-Simons field theory, as described in the last contribution to this thesis) from this point of view. 


\section{CHAPTER 3: Parafermions and Coset Models}

Coset models seem to play a central role in the classification of rational conformal field theories. Although their lagrangian has been known for a long time, little has been done to understand even thier classical structure via these lagrangians. The lagrangians for the coset models we will consider in these next two papers are the gauged Wess-Zumino-Witten lagrangians. Stasting from these lagrangians, the following papers show how to derive the Poisson bracket of the coset models.

In this semiclassical treatment the Poisson structure is neatly represented in terms of "classical parafermion" fields. Indeed we show that for the abelian coset the Poisson algebra indeed closes on these classical parafermions and that for the non-abelian coset the Poisson structure closes on the space of all colorless (with respect to the action of the subgroup) operators built out of the classical parafermions. Other highlights include underst anding the coset models as special types of non-linear $\sigma$ models and also finding a free field realization of the Poisson structure. Also some initial attempts are made to understand what we can learn about the full quantum theory by directly analyzing these lagrangians. 


\title{
Parafermions from Coset Models
}

\author{
K. Bardakci \\ and \\ M. Crescimanno \\ Department of Physics \\ University of Califormia \\ Berkeley, California 94720 \\ and \\ Lawrence Berkeley Laboratory \\ E. Rabinovici \\ Laurence Berkeley Labontory \\ and \\ Racah Tnstitute of Physics \\ Hebrew University \\ Jerusalem 91904, Jsrael
}

in Nuclear Physics B, Vol. 344 (1990), pg. 344 


\section{Introduction}

The construction of two dimensional conformally invariant field theories is a open problem of mathematical physies with applications to statistical mechanics in two dimensions and string theory. Although no general method is 2 yet known, a number of interesting special constructions bave been proposed. ${ }^{1-6}$ One of the oldest methods is the caset construction, which can be thought of as a natural generalization of the Sugawara construction. Historically these models have been formulated ad hoc thus it is clearly desirable to have a more systematic approach to them. This paper espouses a completely lagrangian viewpoint of the coset construction. In a seminal paper Witten showed that the Wess-Zumino-Witten (WZW) lagrangian is conformally intariant, has an underlying affine current algebra and that it correctly implements the Sugawara construction. ${ }^{7}$ It was later shown that the coset models also have a lagrangian basis, obtained by "gauging" an appropriate subgroup of the WZW lagrangian. The corresponding gauge field is not dynamical but acts as a lagrange multiplier to project out the currents belonging to a particular subalgebra. If these model: are quantized in axial gauges, they can be written as a direct sum of several WZW models and hence are clearly conformally invariant. ${ }^{\text {s-11 }}$

In this paper we will investigate the gauged WZW model in the physical (unitary) gauge. For simplicity, we will restrict ourselves to gauging an abelian subgroup. although our methods generalize to non-abelian subgroups. We hope to treat the non-abelian case fully in a subsequent paper. In the pure (non-gauged). WZW model, it is well known that formulating the model in terms of currents leads to great simplifications. In particular. choosing light come coordinates one finds two sets of conserved currents; one set independent of the coordinate $x_{+}$and the other independent of $x_{-}$. Fundamentally this is a consequence of conformal invariance. Furthermore, these currents satisfy simple commutation relations (i.e. they form an affine algebra). Following this lead we search for conserved currents in the gauged WZW model and try to formulate the model entirely in terms of these currents. Then 
the usual local currenis will be gauge dependent and therefore only covariantly conserved. The basic idea of the paper is to build a new set of gauge invariant currents which are truly conserved by attaching a Wilson line to the old gauge covariant currents. These new currents are therefore conserved and gauge invariant but non-local. It is thene new currents that we call "classical parafermions". They are reminiscent of the usual parafermions although these were first introduced in a somewhat different context. ${ }^{12}$

Next we study the commut ation relations between parafermion fields. Since parafermion fields are non-local, equal light-cone time commutation relations between them are illdefined and must be replaced by the more general concept of operator product expansions. Our approach to this problem is a classical one; before quantizing the theory we compute the Poisson brackets of the parafermion fields. This is a well-defined but laborious calculation, to which the bulk of the paper is devoted. We also point out several simplifications which make the calculation for a general group feasible. The resulting Poisson brackets between parafermion fields are quite simple. They are the classical analogue of the operator product expansion. These Poisson brackets are also satisfied by suitably defined functions of free fields. This provides a map (classically) from the original gauged WZW model to a free field theory. In particular we recover the classical limit of the Wakimoto-Nemeschansky construction. ${ }^{13-15}$ Once the theory is expressed in terms of free fields, it is possible to go beyond the classical theory and define various expressions by normal ordering operator products and introducing appropriate (finite) renormalization constants.

The coset model of $S U(2) / U(1)$ is especially interesting. It is one of the very simpllest of cosets and will be discussed at lenght in this paper. In the unitary gauge this model reduces to a $\sigma$-model different than that expected for the coset on geometric grounds, and is not just an $S^{2}(=S U(2) / U(1)) \sigma$-model. To distinguish this $\sigma$-model from the vector coset $\sigma$-model we call them chiral coset (c.c.) $\sigma$-models. These c.c. $\sigma$-models have curvature and therefore non-zero beta function. The mapping described in the preceding 
paragraph maps this theory into a free fieid theory which is conformally intariant. This appears paradoxical since the beta function is not preserved in this mapping. However since the mapping is non-local there is no real paradox, only local field transformations preserve the beta function. ${ }^{16,17}$ Incidentally, this mapping provides a classically exact solution for the $S U(2) / U(1)$ c.c. $\sigma$-model.

The last section of the paper is speculative and deals with issues which go beyond the classical limit. We discuss the $Z_{k}$ grading of the parafermions of level $k$ and we also construct soliton solutions in $S U^{i}(2) / L^{\prime}(1)$ c.c. $\sigma$-models tentatively identifying them with parafermions. We also discuss the possible connection with Feigen-Fuchs-ike constructions ${ }^{18}$ and the connection between screening charges and a natural $\Theta$ parameter that appears in our treatment. 


\section{The Model}

For the sake of cornplateness we now briefly review the cont construction. We begin with the Wess-Zumino-Witten action,

$$
I W Z W=\frac{k}{4 \pi} \int d^{2} x \operatorname{Tr}\left(\partial_{+} g^{-1} \partial_{-g}\right)+\frac{k}{6 \pi} \int \operatorname{Tr}\left(g^{-1} \partial_{+} g g^{-1} \partial_{-} g g^{-1} \partial_{x} g\right) d^{3} x
$$

where $x_{ \pm}=x_{0} \pm x_{1}, \partial_{ \pm}=\frac{1}{2}\left(\partial_{0} \mp \partial_{1}\right)$ and where $g \epsilon \mathcal{G}$ is a be group-valued field, $T$ is taken in some representations of $Q$, the be algebra of $G$. (Tr is normalized so that $\operatorname{Tr}\left(\tau^{a} \tau^{b}\right)=2 \delta^{a b}$ ). By well known arguments, the coefficient $k$ is integer. ${ }^{7}$ As was shown in reference, ${ }^{7}$ the currents g nerated by the symmetry $g \rightarrow A\left(x_{-}\right) g B\left(x_{+}\right)$, where $A, B$ are $G$-valued fields, satisfy" the $\mathcal{G}_{k}$ (called " $\mathcal{G}$ level $k^{*}$ ) Kac-Moody" algebra.

It was known early on ${ }^{1.9}$ that "removing" components lying in a lie-subalgebra of the currents above (i.e. by simply setting them formally to zero) yields a new representation of the Viragoro algebra. One way to remove certain components of the $\mathcal{G}$-currents is by introducing lagrange multiplier fields that, upon path-integration, set the specified components to zero. Since this technique is well known let us discuss the particulars of the model under study. As discussed in ref. $[9,10,11]$ one starts with $2 \mathcal{G}_{k}$ theory, identifies a vector subgroup $\mathcal{H \subset G}$ and introduces an $h$-valued ( $h$ denotes the lie algebra of $H$ ) gauge field $A_{ \pm}$that transforms contragrediencly to the vector $\mathcal{G}_{k}$ currents we wish to project out. For the lagtangian in eq. (2.1) the "left" and "right" currents are

$$
j_{+}=\frac{i k}{\pi} g^{-1} \partial_{+} g \quad j_{-}=-\frac{i k}{\pi} \partial_{-g} g^{-\downarrow} .
$$

Under the subgroup $\mathcal{H}^{\prime}$ s action ( $B \in \mathcal{H}, B$ an arbitrary constant element) $g \rightarrow B^{-1} g B$, the $j_{ \pm}$transform by $j_{ \pm} \rightarrow B^{-1} j_{ \pm} B$ and therefore it is useful to introryce fields $A_{ \pm}$such that under this action $A_{ \pm} \rightarrow B^{-1} A_{ \pm} B$ so that $\operatorname{Tr}\left(A_{+} j_{-}+A_{-} j_{+}\right)$is invariant. In order to set the $\boldsymbol{H}$-components of the $j_{ \pm}$to zero at every point we must promote the $\mathcal{H}$-action described 
above to a local symmetry. One then finds the gauge invariant Jagrangian,

$$
\mathcal{L}=\mathcal{L}_{W Z W}+\frac{i k}{2 \pi} \operatorname{Tr}\left(A_{+} \partial_{-} g g^{-1}-A_{-} g^{-1} \partial_{+} g+A_{+} g A_{-} g^{-1}-A_{+} A_{-}\right) .
$$

Varying $g$ by gauge transformations described above we find that the theory has the currents

$$
\begin{aligned}
& J_{+}=\frac{i k}{\pi} g^{-1} D_{+} g \\
& J_{-}=-\frac{i k}{\pi}\left(D_{-} g\right) g^{-1}
\end{aligned}
$$

where $D_{ \pm}=\partial_{ \pm}-i\left[A_{ \pm}\right.$. Sow using the equations of motion one sees that the $\boldsymbol{h}$ components of these currents vanish. i.e. $\operatorname{Tr}\left(h^{a} J_{ \pm}\right)=0 \quad \forall h^{a} \epsilon \tilde{n}$. Thus

$$
\begin{aligned}
A_{+}^{a} & =\frac{1}{2}(1-M)_{a b}^{-1} \operatorname{Tr}\left(g^{-1} \partial_{+} g h_{b}\right) \\
-A_{-}^{a} & =-\frac{1}{2} \operatorname{Tr}\left(\partial_{-} g g^{-1} h_{b}\right)(1-M)_{b a}^{-1}
\end{aligned}
$$

where the matrix $M$ is defined by.

$$
2 \mathrm{M}(g)_{a b}=\operatorname{Tr}\left(g^{-1} h_{b} g h_{a}\right) \quad \forall h_{a}, h_{b} \hbar
$$

with sum over repeated indices implied and ao distinction is made between raised and lowered indices. Note that $M(g)^{\mathfrak{t}}=\mathbf{M}\left(g^{-1}\right)$ and that $(1-M)$ is not an invertible matrix iff $g \in \mathcal{H}$. That is, for $g \in \mathcal{G} / \mathcal{H}$ the matrix $(1-M)$ will be invertible. Note also that these $A_{ \pm}$(eq. (2.5)) are the solution of the equations of motion found by varying the action of the lagrangian of eq.(2.3) with respect to $A_{ \pm}$. Other equations of motion for this lagrangian tell us that the currents in eq.(2.4) are covariantly conserved,

$$
D_{-} J_{+}=0=D_{+} J_{-}
$$

The fact that these currents $d_{ \pm}$are covariantly conserved (eq(2.7)) and don't have any component in the subalgebra $\hbar$ (eq.(2.5)) leads one to surmise that they are functionally 
related to the parafermions. This intuition turns out to be more or less correct. We will discuss this at length in the next section.

Finally, to close this section, notice that for arbitrary $A_{ \pm}$we have the following useful identity

$$
g\left(D_{-} J_{+}\right) g^{-1}+D_{+} J_{-}=\frac{i k}{\pi}\left[F-g F g^{-1}\right]
$$

where $F$ is given by

$$
F=\partial_{+} A_{-}-\partial_{-} A_{+}-i\left[A_{+}, A_{-}\right]
$$

This identity is analogous to that of the ungauged WZW model in which one finds

$$
g\left(\partial_{-} j_{+}\right) g^{-1}+\partial_{+} j_{-}=0
$$

It was this identity in the ungauged WZW model that ensured the conservations equations for the right and separately for the left moving currents were actually the same equation. In the gauged case, eq.(2.8) evaluated using eq.(2.7) implies

$$
F=0
$$

where we have used the fact that $(1-M)$ is invertible for a generic choice of $g$. This result will be used in the next section in which we identify the classical parafermions. In computing Poisson brackets, however, one wishes to compute the full variation of the action (i.e. with fields "off-shell") and cannot use the equations of motion. However, in section VI and section VII in discussing the semiclassical treatment of the classical parafermions we often use $F=0$. 


\section{ill. The Clarsical Parafermions}

Rather than trying to canonically quantize the action of eq.(2.3) directly we have the freedom to first perform canonical transiormations on the classical fields before identifying the operators which we wish to canonically quantize. Note however that, as usual, this procedure may yield a quantum theory differeut from that found by actually trying to canonically quantize the action eq. (2.3) directly; i.e. classical canonical transformations may be nonunitary when implemented quantum mechanically. This is an important philosophical point of this exposition. Our aim is to form composite operators, $\psi$, of the original degrees of freedom whose Poisson bracket look like those expected of free parafermions. For example. we expect the Poisson algebra of the $\psi$ 's to close, and therefore that the Poisson bracket of two $\psi$ 's is expressible completely in terms of $\psi$. We will then write down free field (Bosonic) lagrangians which furnish a representation of the Poisson algebra of the $\psi$ 's. This allows one to consider canonically quantizing the theory of the $\psi$ 's by writing them as composite operators of free bosons. In a sense this procedure can be carried out for any field theory by inverting the LSZ relation between the full (interacting) quantum field and the free field. In practice however, actually trying to invert the LSZ relations is impossibly unwieldy and involves nonlocal expressions. However for the theory of lagrangian eq.(2.3) there is a simple way of "discovering" the parafermion as 3 composite and mildly nonlocal operator of the fields.

In order to motivate this further, consider what properties we expect the parafermions to possess. In particular, we expect:

1) Gauge invariance; Since we're gauging and performing a functional integral over the gauge field the relevant remaining physical fields should be gauge invariant.

2) Coset-valued field: As described is the introduction the functional integral over the gauge field should project out all components of fields in $\boldsymbol{n}$. Thus $\psi$ representing thw 
remaining degrees of freedom should be coset-valued i.e. have components in $s \backslash h$ only.

3) Conservation The expected conformal nature of the underlying coset model suggests that the currents representing the coset model's physical degrees of freedom be separable in to holomorphic and antiholomorphic pieces. Thus we expect $\psi$ 's (which represent these currents) to depend on either only $x_{+}$or only $x_{-}$e.g. for the left handed field $\dot{\psi}$ we require $\partial_{+} \psi^{i}=0$.

These characteristics strongly suggest that the classical parafermions $\psi$ is related to the coset currents, $J$, of the previous section (see eq.(2.4)). But $J$ is not gauge invariant and is not conserved but is only covariantly conserved (see eq.(2.7)). The obvious choice for $\psi$ is:

$$
\psi=-U^{-1} J_{-} U
$$

where $U$ is the Wilson line

$$
U(x)=P e^{i \int_{x_{0}}^{x} A+d x+}
$$

where $P$ stands for path ordering of the exponential in eq.(3.2). Note that since $J_{-}$is coset valued $\psi$ is as well. Also, $\psi$ is gauge invariant at $x$ and eq.(2.7) may he written $\partial_{+} i^{\circ}=0$. Because it has a Wilson line dangling from it, $\psi$ is nonlocal. This $W$ ilson line gives $v$ unusual statistics. Furthermore the base point $\left(x_{0}\right)$ dependance of $U$ and therefore of $\leftarrow$ introduces further subtleties. Both of these matters are discussed in much more detail in the appropriate places in later sections.

For the purpose of computing Poisson brackets of $\psi$ 's the form of $U$ must be modified. Namely, $U$ as defined in eq.(3.2) involves integrating along a time-like line and $\psi$ will thus be a functional of the field $A_{+}$'s history. This would make the direct computations of the Poisson bracket between two $\psi$ 's problematic. Instead, using eq.(2.i0) $F=0$ we may 
motivate rewriting $U$ in terms of equal. time quantities as;

$$
U(x)=P e^{i \int_{x_{0}}^{*} 1-d x-}
$$

from now on we refer to $\psi$ as defined in eq.(3.1) with $U$ of eq.(3.3). In section $V$ for computing the Poisson bracket of two $\psi$ 's we will retain $\psi$ as defined with $U$ of eq.(3.3) but will not assume $F=0$. 


\section{Poimon Bracket Generalities}

The lagrangian of the gauged WZW model eq.(2.3) has the very important property that it involves terms at most linear in $\partial_{+}$. Regarding $x_{+}$as (light cone) "time" we now analyze this theory classically. Rather than studying the theory's Hamiltonian directly we make use of the canonical method for identifying conjugate variables of a theory whose lagrangian is first order in time derinatives. Let's now sketch this standard procedure, retaining ref.[7] for further reference. Imagine a theory whose actions in given in eq.(4.1).

$$
I=\int A_{i}(\phi) \frac{d \phi^{i}}{d t} d t
$$

where the $\phi^{i}$ are dynamical fields. The above lagrangian contains terms only first order in time derivatives. Varying the fields in an arbitrary way $\phi^{i} \rightarrow \phi^{i}+\mathbf{d} \phi^{i}$ we find

$$
\delta I=\int \mathrm{d} \phi^{j} F_{i j} \frac{d \phi^{i}}{d t} d t
$$

with $F_{i g}$ being an antisymmetric matrix defined by;

$$
F_{i j}(\phi)=\frac{\partial A_{i}(\phi)}{\partial \phi^{j}}-\frac{\partial A_{j}(0)}{\partial \phi^{2}}
$$

Note that since the variation of $I$ is essentially a quadratic form in $d \phi^{i}$ we can use $F_{i j}$ to define the Poisson bracket between the $\phi^{i}$ 's;

$$
\left\{d \phi^{i}, d \phi^{j}\right\}=\left(F^{-1}\right)^{j i}
$$

if $F^{-1}$ is not defined we must choose a subspace of the $d \phi^{i}$ on which $F$ is nonsingular. This subspace is that of the pbysical degrees of freedom. For the case we wish to study, the gauge symmetry of our action makes $F$ singular and simply fixing gauge to a unitary (physical) gauge ensures F's invertibjlity. Note furthermore that we may use the symplectic form $F^{-1}$ of eq. (4.4) for computing the Poisson brackets of arbitrary functions of fields as follows: let $P(\phi), Q(\phi)$ be two arbitrary functions of the fields $\phi^{i}$. To compute their Poisson bracket we 
use the following one-forms,

$$
\mathbf{P}(\phi)=\frac{\partial P}{\partial \phi^{i}} \mathrm{~d} \phi^{i} \quad Q(\phi)=\frac{\partial Q}{\partial \phi^{i}} \mathrm{~d} \phi^{i}
$$

and then by definition

$$
\{\mathbf{P}, \mathbf{Q}\}=\frac{\partial P}{\partial \phi^{i}} \frac{\partial Q}{\partial \phi^{j}}\left\{d \phi^{i}, d \phi^{j}\right\}=\frac{\partial Q}{\partial \phi^{j}}\left(F^{-1}\right)^{j i} \frac{\partial P}{\partial \phi^{i}}
$$

We will often need to compute the Poisson brackets of arbitrary one-forms

$$
V=V_{i} d \phi^{i} \quad W=W_{i} d \phi^{i}
$$

these forms are not necessarily closed or exact and we will define their Poisson bracket to be

$$
\{\mathbf{V}, \mathbf{W}\}=W_{i}\left(\mathbf{F}^{-1}\right)^{i j} V_{j}
$$

thus their Poisson bracket is a linear cormbination of Poisson brackets of the basis one-forms.

Let's now return to the theory of the gauged WZW' model whose lagrangian is given by eq.(2.3). Agajn, we imagine having already performed the integrations over $A_{ \pm}$so for the $A_{ \pm}$in eq.(2.3) we substitute eq.(2.5). If we now compute the variation of the action with respect to the arbitrary variation $g \rightarrow g+\delta g$ we find,

$$
\delta I=\int d^{2} x \operatorname{Tr}\left[g^{-1} \delta g\left(D_{-} J_{+}+\frac{i k}{\pi} F\right)\right]
$$

where $F$ is that of eq. $\{2.9\}$. We will regard $\delta g$ as an arbitrary variation and when we wish to imagine having chosen a particular co-ordinate system of $\mathcal{G}$ we will think of $\delta g$ as a form dg. Note that $J_{+}=\frac{i k}{\pi} g^{-1} D_{+} g$ is a lie algebra element orthogonal to $\hbar$ i.e. "pointing into the cocet" and that furthermore $D_{-} J_{+}$points also into the coset. The second term of eq. (4.8), $F$, of course lies entirely in $\boldsymbol{h}$.

Since we wish to compute Poisson brackets in this theory but using the machinery presented in the previous paragraphs, we must write the variation of the gauged WZW 
action given in eq. (4.8) in a form more similar to that of eq.(4.2). Note that in eq.(4.2) $\delta I$ is written as an antisymmetric (in all indices) form and is antisymmetric under the interchange of $\frac{d \phi^{i}}{d t}$ and $d \phi^{j}$. We now will recast eq.(4.8) in a form in which this antisymmetry is manifest. To this end define

$$
\delta A_{b}=\frac{1}{2}(1-M)_{b_{0}}^{-1} \operatorname{Tr}\left(g^{-1} \delta g h^{a}\right)
$$

where $h^{a}$ are the generators that span $h$. Then, concentrating on the second term of eq.(4.8) we have.

$$
\operatorname{Tr}\left(g^{-1} \delta g F\right)=-2 \operatorname{Tr}\left(\delta A(2-M)^{t} F\right)
$$

which, using the property that $\mathbf{M}^{c}(g)=\mathbf{M}\left(g^{-1}\right)$, is

$$
\operatorname{Tr}\left(g^{-1} \delta g F\right)=-\operatorname{Tr}\left(\delta A\left(F-g F g^{-1}\right)\right)
$$

Now using the identity eq. (2.8) and the fact that $D_{+} J_{-}$points only into the coset (and so is orthogonal to $\delta . A$ in the lie algebra $\ell$ ) we have;

$$
\operatorname{Tr}\left(g^{-1} \delta g F\right)=\frac{\pi i}{k} \operatorname{Tr}\left(g^{-1}[\delta A, g] D_{-} J_{+}\right)
$$

and thus eq.(4.8) may be recast as;

$$
\delta I=\frac{k}{2 \pi} \int d^{2} x \operatorname{Tr}\left[g^{-1}\left(\delta g-i\left[\delta A_{,} g\right]\right)\left(\partial_{-}-i\left[A_{-},\right) g^{-1}\left(\partial_{+} g-i\left[A_{+}, g\right]\right)\right] .\right.
$$

Note this expression is of a form similar to that of eq.(4.2) and it is this form of $\delta I$ that will be used for the bulk of the ensuing computation. Again, the operator $D_{-}$is not invertible unless we fix the gauge completely.

The variation of the action eq.(4.13) is for a general WZW caset model. In the next section we will study the abelian coset model (the case in which $\mathcal{H}$ is abelian) in detail and leave the applications of eq. (4.13) to nonabelian cosets ( $\mathcal{H}$ nonabelian) for future work. 


\section{Poiseon Brackets of the Abelian Coset}

We asw specialize to the case of abelian cosets. By abelian coset we mean that $\mathcal{H}$, the subgroup being "mojded" out, is a subgroup of the torus of $G$. Thus $h$ is a subalgebra of the Cartan subaigebra. To begin computing physically meaningful quantities we imagine completely fixing gauge. Although an explicit form of gauge fixing will not be necessary for what follows we imagine completely fixing gauge with a unitary gauge condition $f(g)=0$ where $f$ involves ao spatial derivatives. Thus the remaining degrees of freedom will be physical. Choosing such a gauge has it's subtleties, particularly with lagrangians that have "topological terms" as that of the gauged WZW model and we will discuss some of these matters later. For the present discussion we ignore these subtleties.

For the purposes of this calculation it is necessary to choose co-ordinates on $\mathcal{G}$. Since we have fixed gauge it will be particularly convenient to choose co-ordinates that naturally "stay" in this chosen gauge. We now use this notion to motivate a particularly useful set of co-ordinates on $\mathcal{G}$. As noted earlier, the action of the gauged WZW model has a global symmetry under $g \rightarrow A g B$ with $A, B \in \mathcal{G}$. Part of this $\mathcal{G} \times \mathcal{G}$ chiral symmetry then gauged

i.e. a particular vector subgroup of $\mathcal{G} \times \mathcal{G}$ given by $g \rightarrow U g U^{-1}$ with $U \in \mathcal{H}$ was made a local symmetry of the action. The axial global symmetry $g \rightarrow B g B \quad B \in \mathcal{H}$ is still a good symmetry of the action and furthermore will not take $g$ out of the chosen unitary gauge. Thus co-ordinates associated with this axial symmetry are a natural choice. Keeping this in mind ve can always decompose $g$ as;

$$
g=C B g B C^{-1}
$$

where $B, C \in \mathcal{H}$ and $\bar{g} \in \mathcal{G}$ is constrained in the following way; set $C=1$ (since jt corresponded to the gauged $\mathcal{H}$ invariance) and let $g$ satisfy the unitary (e.g. physical) gauge conditions deacribed in the bezinning of this section, leaving BcK arbitrary. This forces $\bar{g}$ to depend on $|\mathcal{G}|-2|\mathcal{H}|$ independent co-ordinates (the $|\mathcal{G}|$ and $|\mathcal{H}|$ are the dimensions of $\mathcal{G}$ and $\mathcal{H}$ 
respectively), $\rho^{l}$, in some nontrivial way. Fortunately an explicit form of $\bar{g}$ is not necessary for further computation. To fix notations and conventions let $B=e^{i 8 h^{*}}$ with $h^{\alpha} \in h$, with $\left\{h^{a}\right\}$, as before, forming a suitable basis of $h$, and $\theta^{\infty}$ being real-valued parameters. Furthermore we will think of $\bar{g}$ as $e^{i \rho^{t} \tau^{t}}$ where $\tau^{l} \epsilon q \mid h$ span the vector space of $Q$ orthogonal to $\pi$. We will indicate a coret index by $l, m, n$ and an $h$ index $a, b, c$. In these co-ordinates a general variation of $g$ may be written

$$
\delta g=\frac{-i}{2}\left\{h^{a}, g\right\} d \theta^{a}+K^{\ell} d \rho^{l}
$$

where $\{$,$\} is an anticommutator. Again note that this variation keeps g$ in the chosen gauge. The particular form of $K^{\ell}$ win not be needed in the rest of the calculation.

We are now ready to compute Poisson Brackets of fields in the gauged WzW model. Instead of computing the Poisson brackets of just any quantities ${ }^{20}$ we follow the intuition of section III and concentrate our interest on the Poisson brackets of $\psi$ 's, the classical parafermions. First note that it is simple to verify that left moving and right moving parafermions' Poisson bracket is zero.

Note that unlike the case of the ungauged WZW model, ${ }^{7}$ the selevant conserved cutrents, e.g. the $\psi$ 's, cannot be expressed directly in terms of the forms that appear in eq.(4.13). Thus it will be necessary to compute Poisson brackets of several other quantities in addition to that of the forms that appear in eq.(4.13). In particular we need the Poisson bracket between $F$ of eq. (2.9) and the co-ordinates $\theta^{\infty}, \rho^{\ell}$. Indeed, using eq.(5.2) in eq.(4.8) (which is simply another form of eq.(4.13)) we find that

$$
\delta I=\frac{k}{\pi} \int d^{2} x \mathrm{~d} \theta^{a} \operatorname{Tr}\left(h^{a} F\right)+\ldots
$$

where .... stands for terms involving $d \rho^{\ell}$. By $d \theta^{\circ}$ we mean $d \theta^{\circ}$ considered as a form in the tangent space of co-ordinates $\theta^{\infty}, \rho^{\ell}$ as described in Section IV. Note that this expression implies $F=0$ as an equation of motion, completely consistent with the analysis of Section 
II. Equation (5.3) implies the followizg Poieson brackets:

$$
\begin{aligned}
& \left\{\mathrm{d} \theta^{a}(x), F^{b}(y)\right\}=\frac{\pi}{k} \xi^{a b} \delta(x-y) \\
& \left\{\mathrm{d} \rho^{\ell}(x), F^{b}(y)\right\}=0
\end{aligned}
$$

Note that the second Poisson bracket is the renult that $\theta$ and $\rho$ are independent parameters and that eq. (5.3) is the variation of the whole action. By this we mean $F$ that appears in the first term is eq.(5.3) considered as a form $F$ (for the purpose of computing it's Poisson bracket) in the tangent space to $\theta, \rho$ co-ordinate space can be written in terms of the inverse of the symplectic form. Then eq, (5.4) follow from computation of the Poisson bracket using eq.(4.6) in summary, the content of these equations is that $\theta^{a}$ is canonically conjugate to $F^{\circ}$. Thus, to compute the Poisson bracket of any guantity with $F$,we need only compute that quantities variation with sespect to $\theta$ and do not need to concern ourselves with its variations with respect to the $\rho^{l}$ (all the other co-ordinates). This is the reason we'll never need to know the precise form of the $K^{\ell}$ of eq. (4.2). Extensive use of this observation is made in the computation of Poisson brackets.

As pointed our earlier, the classical parafermion, $\psi$, has several important properties; $\psi$ is coset-valued, gauge-invariant and satisfies simple "free-fielli" equations of motion (see description of $\psi$ in Sections III). For the abelian covet $\psi$ of eq.(3.1) may be written

$$
\psi(z)=-e^{-i x} J_{-} e^{i x}
$$

where

$$
x=\int_{-\infty}^{x} A-d x
$$

The parafermion $\psi$ is really a bilocal field siuce the Wilson line depends on the basepoint $x_{0}$ of integration in eq.(5.6). Thus, technically speaking, it appears that $\psi$ is not even a gauge invariant field since under gauge transformations at $x_{0}, \psi$ will transform homogeneously. 
This subtlety proves to be no obstacle for computing Poissor. brackets of the abelian coset but demonstrates a critical difference between the abelian and nonabelian cosets. This point will be discussed further in the last section.

This subtlety aside, we see that the parafermion $\psi$ is gauge invariant, conserved on-shell and is a non-local functional of the field $g$ (namely $x$ is nonlocal). Computing the Poisson bracket of two $\psi$ 's directly is cumbersome and so, in order to simplify the calculations, it is useful to write $\psi$ entirely in terms of gauge-invariant quantities. To this end define the following gauge fnvariant quantity;

$$
f=e^{-i x} g e^{i x}
$$

in terms of $f$ the classical parafermion $\psi$ of eq. (5.5) has the following simple form

$$
\psi=\frac{i k}{\pi}\left(\partial_{-} \rho\right) f^{-1} \text {. }
$$

Now, as described earlier, in order to compute $\psi$ 's Poisson brackets we would, in general. need to know it with respect to coordinates $\theta^{\circ}$ and $\rho^{l}$, Inder an arbitrary variations of $g$. $\psi$ changes by

$$
d \psi=\frac{i k}{\pi} f \partial_{-}\left(f^{-1} d f\right) f^{-1}
$$

where, written in co-ordinates $\theta^{e}, \rho^{\ell}$ the $d f$ is;

$$
d f=\frac{-i}{2}\left\{f, h^{\alpha}\right\} d \theta^{\infty}+[f, d x]+\ldots
$$

where $\{$,$\} is an anticommutator and ... here represent terms involving d \rho^{\ell}$. At this point it is important to remark that for the rest of the calculation we will only need to know the $d \theta^{a}$ terms of $d f$ explicitly. the $d \rho^{\ell}$ terms Poisson brackets will be borne out automatically: in the calculation. This is a technical point which is explained more fully in the appendix.

It is also convenient to write $\delta I$ of eq.(4.13) in terms of $f$ and other gauge-invariant quantitjes. One finds,

$$
\delta I=\frac{k}{2 \pi} \int d^{2} \approx \operatorname{Tr}\left[f^{-1}(\mathbf{d} f+i[\mathbf{H}, f]) \partial_{-}\left(f^{-1}\left(\partial_{+} f+i[H, f]\right)\right)\right]
$$


where $H=\int d x_{-}^{\prime} \frac{1}{2} \epsilon\left(x-x^{\prime}\right) F\left(x^{\prime}\right)$ and $F$ is given by eq.(2.9) specializes to the abelian case and $\epsilon\left(x-x^{\prime}\right)$ is the antisymmetric $\theta$-function,

$$
\epsilon\left(x-x^{\prime}\right)=\left\{\begin{array}{cl}
1 & x>x^{\prime} \\
-1 & x<x^{\prime}
\end{array}\right.
$$

$A$ comment is in order by way of explaining $d f$ and $H$. In keeping with the discussion of section III describing the use of forms for facilitating the generalized Poisson bracket, we regard $d f$ and $\mathrm{H}$ as forms in the tangent space of the parameters $\theta^{\infty}, \rho^{\ell}$. In this notation $\mathrm{d} f$ is a closed form that may be regarded as a linear combination of closed forms $\mathrm{d} \theta^{\circ}, \mathrm{d} \rho^{t}$ In this same sense by $\mathbf{H}$ we mean the form found by replacing all the $\partial_{+} g$ in the expression for $\boldsymbol{H}$ by $\mathrm{d} g$. More explicitly said, $\mathrm{H}$ is

$$
\mathbf{H}=\boldsymbol{x}-\mathbf{A}
$$

where $A$ is as defined in eq.(4.9) but where $\delta g$ has been repiaced with $\mathrm{d} g$ and by $x$ we mean tin the expression $\int^{x} \partial_{+} A_{-} d x_{-}$replace $\partial_{+} g$ with $d g^{n}$. With $\delta I$ now written in terms of these forms we will employ the generalization of the Poisson bracket described in section IV.

Now simply defining $R=f^{-1}\left(d f+i[H, \Omega)=R^{\ell} \tau^{\ell}\right.$ and using eq. (5.11) we have the following Poisson bracket;

$$
\left\{\mathbf{R}^{\ell}(x), \mathbf{R}^{m}(y)\right\}=-\frac{k}{2 \pi} \delta^{\ell m} \epsilon(x-y) .
$$

As is obvious comparing the form $\mathbf{R}$ with : bat of $d \psi$, the $d \psi$ cannot be expressed entirely in terms of $\mathbf{R}$. Thus in computing the Poisson bracket between two t's we will need to know the Poiscon bracket between $\mathrm{d} f$ and $\mathbf{H}$, etc. Using eq. (5.4) and the definition of $\mathbf{H}$ we have

$$
\begin{aligned}
& \left\{\mathrm{d} \theta^{\circ}(x), \mathrm{H}^{b}(y)\right\}=-\frac{\pi}{2 k} \delta^{a b} \epsilon(x-y) \\
& \left\{\mathrm{d} \rho^{l}(x), \mathrm{H}^{b}(y)\right\}=0 .
\end{aligned}
$$


The other manipulations necessary to compute the Poisson bracket between two $w$ 's is straight forward but tedjous and is detailed in the appendix. Here we present the final result;

$$
\begin{aligned}
\left\{\psi^{L}(x), \psi^{m}(y)\right\} & =\frac{2 \pi}{k} f^{\alpha \mu^{\prime}} f^{a m m^{\prime}} \psi^{c}(x) \psi^{m^{\prime}}(y) c(x-y) \\
& +i f^{\ell m n} \psi^{n}(x) \delta(x-y) \\
& -\frac{k}{\pi} \delta^{\prime}(x-y) \delta^{\ell m}
\end{aligned}
$$

where. as before the index a runs only over generators is $\pi$ and $\ell, m, \ell^{\prime}, m^{\prime}$ run only over coset directions. The $f^{a i l^{\prime}}$ are, of course. the structure constants of the Lie algebra $f^{\text {ale }}=\frac{1}{2} \operatorname{Tr}\left(h^{\mathrm{a}}\left[\tau^{\ell}, \tau^{\ell^{t}}\right)\right)$ with $h^{\mathrm{a}} \in \boldsymbol{h}$ and $\tau^{\ell}, \tau^{\ell} \in g \mid h$. Note that, as expected, the Poisson bracket of eq. (5.16) does indeed satisfy the Jacobi identity. The Jacobi identity was checked by hand and makes critical use of the fact that $h$ is an abelian subalgebra. Thus we've learned that the classical Poisson algebra of the $\psi$ 's closes. We will show that this algebra may be represented in term of free fields.

We now specialize to the case of $S U(2) / U(1)$ with the convention that the coset currents $\psi_{+}, \psi_{-}$point in the directions $\tau_{-}, \tau_{+}$respectively and that $\tau_{3}$ is the generator of the $C_{(1)}$ subgroup (the C.S.A.) being $\mathcal{H}$. In this simple case $\psi=\psi_{+} \tau_{-}+\psi_{-} \tau_{+}$and now using eq. (5.16) the Poisson bracket of two $\psi$ 's reads

$$
\begin{aligned}
& \left\{\psi_{+}(x), \psi_{+}(y)\right\}=\frac{\pi}{2 k} \epsilon(x-y) \psi_{+}(x) \psi_{+}(y) \\
& \left\{\psi_{+}(x), \psi_{-}(y)\right\}=-\frac{\pi}{2 k} \epsilon(x-y) \psi_{+}(x) \psi_{-}(y)-\frac{2 k}{\pi} \delta^{\prime}(x-y) .
\end{aligned}
$$

Note again that this Poisson bracket closes, and that, since $\psi$ is a "conserved" quantity $\left(\partial_{+} \psi_{ \pm}=0\right)$ and further since the Poisson bracket of conserved quantities is also a conserved quantity, the right hand sides of eq.(5.16) and eq.(4.17) involve simple products of the 4 's only. This is as expected. Indeed all the classically conserved quantities are just polynomials of $\psi_{ \pm}$'s. Thus with this classical approach one cannot conclude what functions of fields 
become the the primary fields of the quantum theory or even surmise how many primary fields there are. These are questions answerable only by actually trying io canonically quantize this theory and studying the composite operators that appear in the operator product expansions.

In analogy with conformal fir' 2 theories' algebraic formulation we define a local "stress"energy tensor for this $S U^{\prime}(2)$ coset by,

$$
T(x)=c \psi_{+}(x) \psi_{-}(x)
$$

(Remember that the \pm ase group indices and that $\psi_{ \pm}\left(x_{-}\right)$are both left handed fields) where $c$ is a constant that will now be determined. The Poisson bracket for $T$ with $\dot{w}_{ \pm}$is;

$$
\left\{T(x), \psi_{ \pm}(y)\right\}=\frac{-2 k}{\pi} c \delta^{\prime}(x-y) \psi_{ \pm}(x)
$$

choosing $c=-\pi / 2 k$ we see that $\psi_{ \pm}$indeed transform as chiral conformal fields. It is now simple to compute the Poisson bracket between two $T$ 's.

$$
\{T(x), T(y)\}=\delta^{\prime}(x-y)\{T(x)+T(y)\}
$$

which is the expected result. Note that the algebra of the $T$ 's contains no central charge. Again this is expected since we are only computing (elassical) Poisson bracket.

To now connect this approach with the Feigen-Fuchs-like construction (at the classical level). We find a free-field representations for the algebra of the $S U(2) / U(1)$ model depicted in eq.(5.17). ${ }^{13-15}$ The fact that this may always be done for a well-defined Poisson bracket is guaranteed by Darboux's theorem. Simply counting degrees of freedom indicates that if it is possible to bosonize the $\psi_{ \pm}$in eq. (5.17) one will need two bosonic fields. Denote these free, two-dimensional bosonic fields by $\phi_{1}, \phi_{2}$. We have

$$
\left\{\mathrm{d} \phi_{\sigma}(x), \mathrm{d} \phi_{\beta}(y)\right\}=\frac{1}{2} \delta_{\alpha \beta \epsilon}(x-y) \quad \alpha, \beta=\{1,2\}
$$


where the $d$ signifies that, as explained earlier, we are thinking of the Poisson bracket as an antisymmetric bilinear form. Now taking a hint from the functional form of the $S U(2) / C(1)$ parafermion (and also knowing the form of the correct answer ${ }^{13-15}$ ) we form the ansatz,

$$
\psi_{ \pm}=B_{ \pm} e^{ \pm i A}
$$

where $B_{ \pm}$and $A$ are yet-to-be-determined local functionals of $\phi_{1}, \phi_{2}$. Now writing out eq. (5.17) we have;

$$
\begin{aligned}
\left\{\mathrm{d} B_{+}(x)+i B_{+} \mathrm{d} \cdot A(x), \mathrm{d} B_{+}(y)+i B_{+} \mathrm{d} A(y)\right\} & =\frac{\pi}{2 k} \epsilon(x-y) B_{+}(x) B_{-}(y) \\
\left\{\mathrm{d} B_{+}(x)+i B_{+} \mathrm{d} A(x), \mathrm{d} B_{-}(y)-i B_{-} \mathrm{d} \cdot A(y)\right\} & =\frac{-\pi}{2 k} \epsilon(x-y) B_{+}(x) B_{-}(y) \\
& -\frac{2 k}{\pi} \delta^{\prime}(x-y)-\frac{2 i k}{\pi} \delta(x-y) \cdot A^{\prime}(x) .
\end{aligned}
$$

Of course one also has the equation similar to eq. (5.23a) but involving $B_{-}$coming from the Poisson tracket of two $\psi_{-}$. Note that choosing $A(x)=-i \sqrt{\frac{\pi}{k}} \phi_{1}(x)$ the terms on the right hand side are quadratic in $B_{ \pm}$. Furthermore, in order to get terms involving $\delta(x-y)$ and $\delta^{\prime}(x-y)$ we need $B_{ \pm}$to be a linear functional of $\phi_{1}$ and $\phi_{2}$ involving derivatives. Thus take for $B_{ \pm}$the following ansatz,

$$
\begin{aligned}
& B_{+}=c \partial \phi_{1}+\tilde{c} \partial \phi_{2} \\
& B_{-}=e \partial \phi_{1}+\tilde{e} \partial \phi_{2} .
\end{aligned}
$$

The Poisson bracket for two $\psi_{-}$'s implies $e^{2}=-\vec{e}^{2}$ and solving for $c, \bar{c}, e, \bar{e}$ by using eq. $(5.23 \mathrm{~b})$ one finds,

$$
\psi_{ \pm}=\sqrt{\frac{k}{\pi}}\left(\partial \phi_{1} \pm i \partial \phi_{2}\right) e^{ \pm \sqrt{\hbar} \phi_{1}}
$$

Let us pause to make some comments concerning this representation. Note that $\phi_{1}$ and $\phi_{2}$ are really complex fields and that $\psi_{ \pm}$are not Bermitian conjugates. Actually since we 
are doing a completely classical analysis there is really no notion of hermiticity, but fields are simply real or complex. In the representation we have chosen the $\psi$ are indeed complex. This is presumably the classical analogue of the quantum result in which ghost fields are employed to construct $\psi^{13-15}$ Note also that this is not quite the same as the full quantum result of ref.[14] and that, although they agree in the (classical) limit $k \rightarrow \infty$, there are (presumably) renormalization effects in the quantum theory at finite $k$ that change result eq.(5.25).

We close this section by noting, a was pointed out in ref.[21], that the functional determinant between the fields that make up the currents of the $S U(2) / U(1)$ model and the free fields used above is nontrivial. It involves spatial derivatives and may be integrated, as was done for example with the conformal anomaly, to yield a crucial term in the quantum effective action. 


\section{Solitons; An Example}

In the previous section the parafermionic currents of the abelian coset were constructed and their Poisson brackets studied. Continuing in the vein of analyzing the theory classically we would like to study solutions to the equations of motion in the simplest possible abelian coset, i.e. that of $S U(2) / U(1)$. To be explicit let $\left\{\sigma_{i}\right\}$ be the Pauli spin matrices in the fundamental of $S U(2)$. A general group element $g \in \mathcal{G}(=S U(2))$ will be written $g=g_{0} 1+i \vec{g} \cdot \vec{\sigma}$ with $\bar{g}=\left(g_{1}, g_{3}, g_{3}\right)$ and with the condition that $g_{\hat{0}}^{\hat{0}}+\vec{g}^{2}=1$. We now choose the $U(1)$ subgroup generated by $\sigma_{3}$ of $S U(2)$ to be the abelian subgroup $\mathcal{H}$ which we are gauging. Strictly speaking both the gauge fields and the currents are in the adjoint representation and so expressions such as $g^{-1} \partial_{ \pm} g$ are interpreted as being in the adjoint even though $g$ is in the furdamental representation. Since the lagrangian of eq.(2.3) has a local gauge invariance under which $g(x) \rightarrow U g U^{-1}(x)$ with $U \epsilon U(1)$ we may choose a gauge slice in which $g_{2}=0$. (By $g_{2}$ we mean the second component of the $\vec{g}$ vector in the definition of the group element $g=g_{0} 1+i \vec{g} \cdot \vec{\sigma}$ ). This can always be done, and furthermore this choice completely fixes the gauge freedom except at points where $g(x)$ is an element of $\mathcal{H}$. This choice of gauge also makes the WZW term equal zero naively. Ostensibly che entire support of the WZW term must come from the points (or lines) on which $g(x) \in \mathcal{H}$. This is a subtle point and one which we will return to in subsequent work. Notice that although this choice of gauge makes the WZW term of $\mathcal{L}$ vanish, the WZW term still effects the definition of the currents and therefore the $A_{ \pm}$. Indeed writing the coset lagrangian of eq.(2.3) in this unitary gauge $\left(g_{2}=0\right)$ and using the $A_{ \pm}$of eq. (2.5) we find,

$$
\mathcal{L}=\frac{k}{4 \pi} \frac{\partial_{\mu g_{0}} \partial^{\mu} g_{0}+\partial_{\mu} g_{3} \partial^{\mu} g_{3}}{1-g_{0}^{2}-g_{3}^{2}}
$$

where we have used the fact that $g_{0}^{2}+\tilde{g}^{2}=1$ to eliminate $g_{1}$. This lagrangian is not that of in $S^{2}=S U(2) / U(1) \sigma$-model, which is the model one would recover from this coset were there no WZW term. This new $\sigma$-model will be referred to as the chiral coset (c.c.) 
$\sigma$-model in order to distinguish it from the ordinary conet $\sigma$-model.

This lagrangian is not manifestly chiral and has a residual global $0(2)$ symmetry on the fields $g_{0}, g_{3}$. Essentially this left-over symmetry is due to the isometry of the coset space and using Noether's theorem we find that the corresponding currents are,

$$
\mathcal{J}_{ \pm}=\frac{k}{2 \pi}\left(\frac{g_{3} \partial_{ \pm} g_{0}-g_{0} \partial_{ \pm} g_{3}}{g_{1}^{2}}\right) \text {. }
$$

Note that these currents bear a striking resemblance to the $A_{ \pm}$or eq.(2.5). Indeed we have,

$$
\mathcal{J}_{+}=\frac{-k}{\pi} A_{+} \quad \mathcal{J}_{-}=\frac{k}{\pi} A_{-}
$$

and so the current consertation condition $\partial_{+} \mathcal{J}_{-}+\partial_{-} \mathcal{J}_{+}=0$ implies $F=0$ (where $F$ is as in eq.(2.9) specislized to the abelian case). This may be trivially generalized to arbitrary abelian coset models. The lagrangian of eq.(6.1) is not conformal in the ordinary sense. Indeed, considered as a $\dot{\sigma}$-model, this lagrangiars has nonzero beta function as it corresponds to a $\sigma$-model with negative curvature. ${ }^{16,17}$ One may wonder how a lagrangian with non-zero beta function could possibly describe a conformally invariant theory. Since this problem is clearly beyond the reach of the semiclassical apprixach used in this paper, we can only speculate about possible resolutions. One possibility is thit the model is conformal when expressed in terms of the parafermion field $\psi$, which is expressible in terms of the original group variables $g$ through a non-local mapping (eq.(5.5)). Non-local mappings are not, in general, expected to preserve the curvature and the beta function. It is therefore quite possible that a model with non-trivial beta function can be mapped into a free field theory through a non-jocal mapping. Anotber possibility is that the gauge fixed form of the lagrangian eq.(6.1), although classicaly correct, receives quantum corrections. It may also be that there are non-perturbative corrections to the beta function that cause it to vanish. These questions are presently under study.

Let us now write the classical parafermions of the previous sections for this model $S U(2) / U(1)$ in terms of $g_{0}$ and $g_{3}$. It is convenieut to use complex notation to represent 
this gauge-fixed $g$. Let $u=g_{0}+i g_{3}$ and $t^{*}=g_{0}-i g_{3}$. The classical parafermion $t_{-}$is then

$$
\psi_{-}=-\frac{k}{\pi} \frac{\partial_{-} u}{1-u^{\bullet} u} \exp \left[-\int \frac{u \partial_{+} u^{\bullet}}{1-u^{*} u} d x_{+}\right]
$$

where we have used the conservation equations $F=0$. The $\psi_{+}$, which completes the $S U(2) / U(1)$ parafermion doublet (recall that here the subscript \pm on $\psi$ is a group index and zot a Lorentz index), is the conjugate of $\psi_{-i}$

$$
\psi_{+}=-\frac{k}{\pi} \frac{\partial_{-} u^{\bullet}}{1-u^{*} u} \exp \left[-\int^{x} \frac{u^{*} \partial_{+} u}{1-u^{*} u} d x_{+}\right]
$$

Notice that these $\psi$ of eq. (5.5) and eq.(5.4) do satisfy the equations of motion expected for free massless fermions $\left(\partial_{+} \psi=0\right)$. Since $\psi_{ \pm}$solve such simple equations of motion it is perhaps difficult to imagine them as nontrivial solutions of differential equations, i.e. as solitons. Let us construct an actual soliton solution to the equations of motions of lagrangian of eq. (6.1). First it is convienent to choose the co-ordinates $6, \phi$ defined by

$$
\begin{aligned}
\sin \theta & =\sqrt{g_{0}^{2}+g_{3}^{2}} \\
\cos \phi & =\frac{g_{0}}{\sqrt{g_{0}^{2}+g_{3}^{2}}}
\end{aligned}
$$

in which lagrangian eq.(6.1) is

$$
\mathcal{L}=\frac{k}{4 \pi}\left(\partial_{\mu} \theta \partial^{\mu} \theta+\tan ^{2} \theta \partial_{\mu} \phi^{\mu} \phi\right)
$$

To look for solitonic solutions consider $\theta, \phi$ to be functions of $x$ only. Notice how similar this lagrangian is to that of a $S^{2} \theta$-model (one would replace $\tan ^{2} \theta$ with $\sin ^{2} \theta$ to get an $5^{2}$-model's lagrangian). Indeed the equation of motion for $\phi$ (which may deduced from current conservation above whose charge generates $\phi \rightarrow \phi+$ const.) is

$$
\partial_{x}\left(\tan ^{2} \theta \partial_{x} \phi\right)=0 \Rightarrow \tan ^{2} \theta \partial_{s} \phi=n \quad n \text { is real and constant }
$$


which when substituted into the equation of motion for $\theta$ yields exactly the $S^{2}$ equations of motion for $\theta$;

$$
\partial_{x}^{2} \theta-\frac{n^{2}}{\tan \theta \sin ^{2} \theta}=0
$$

This has the well known solution

$$
\cos \theta=\sqrt{1-n^{2}} \sin x \text { with } n<1
$$

where because of the scaling freedom in $x$ we have, for convienence, scaled $n$ to be some number less than one. This classical symmetry of the equations of motion is as expected since $\mathcal{L}$ contains no dimensionful parameters.

It is more difficult to solve eq.(6.8) for $\phi(x)$ and here we present only the formula for the advance of $\phi$ as we integrate eq. (5.8) from $x \rightarrow x+2 \pi$. One finds

$$
\Delta \phi=\frac{2 \pi n}{|n|}(1-|n|)
$$

this means that along one "swell" of the soliton the vector $\left(g_{0}, g_{1}\right)$ does not rotate into itself but rather is displaced angularly by $\Delta \phi$ in eq.(6.11). Although this seems strange we are for the moment working in an open topology and so are really interested in how much the phase of $\psi_{ \pm}$advances, not of what happens to the original fields $g_{0}, g_{3}$. We will discuss particulars of a compact topology in the next paragraph. Indeed referring to eq.(6.4) and eq.(6.5) we find an additional contribution to the phase of $\psi$ coming from the exponential factors which when combined with $\Delta \phi$ of eq.(6.11) yields

$$
\underset{x=x+2 \pi}{\text { phase advance of }} \psi_{ \pm}= \pm \frac{2 \pi n}{|n|} \text {. }
$$

Most often one is interested in the gauged WZW model on a compact space. Then single-valuedness of the fields 90,93 requires that the extent of the manifold in $x$, our scaled co-ordinate, is $2 \pi N$ so that referring to eq.(6.11) $n N$ is an integer. Thus we expect $n$ to be rational, and so at fixed compactification the functional integral will get contributions 
from these $N$ solitons. In summary, we have shown that solitons are a plausible candidate for the classical parafermions. 


\section{Semichatical Musinge}

There are, at this point, many questions this approach leaves open. In this section we mention only 2 few things that might prove useful for relating this work with the vast body of literature on the Wess-Zumino-Witten model and conet constructions. The discussion is mostly semiclassical and is not to be regarded as rigorous.

To begin with note that the classical parafermions of eq.(3.1) in the abelian coset are, by construction, invariant under vector $U(1)^{r}$ transformations ( $r$ is some number less than the rank of $\varrho$ ) but under the axial $U(1)^{r}$, generated by the algebra of anticommatators, the $U$ 's acquire extra phases. As an example consider the case $S U(2) / U(1)$. Then global axial transformations $g \rightarrow e^{i \ell n} g e^{+i \ell n}$ lead to

$$
\psi_{+} \rightarrow e^{-i \theta_{+}} \psi_{+} \quad \psi_{-} \rightarrow e^{i \psi_{-}}
$$

where, as usual, the $\psi_{ \pm}$are assumed to be in the adjoint representation. These transformed $\psi_{ \pm}$again satisfy Poisson algebra eq.(5.17). Note that in the Feigen-Fuchs construction in section IV this symmetry corresponds to shifting $\phi_{1}$ (in eq. (5.25)) by a constant. But in the quantum theory one will, in general, be concerned with a Feigen-Fuchs construction (or a WZW model) on some compact space and so the zero-inode of $\phi_{1}$ (the mode $\phi_{1}=$ constant) will be an operator and not allow the above symmetry. Said a different way: the classical symmetry $\phi_{1} \rightarrow \phi_{1}+c, c$ constant, does not survive quantization, i.e. is not representable by a unitary transformation on the Fock space. It is well known that this phenomenon occurs in theories of systems at criticality, such as that of a condenced Bose gas where fermion number is spontaneously broken by the ground state. Also, in a compact space the zero-mode of $\phi_{1}$ will be related to the monodromy of the parafermions and so will take on discrete values. Thus the rigid axial $U(1)$ breaks to some subgroup $\mathbf{Z}_{k}$ and this remaining symmetry is associated with the grading of the parafermionic algebra. ${ }^{12-15} \mathrm{~W}_{\mathrm{e}}$ discuss this point from a different point of view in the next few paragraphs. 
1. Rarafermion statistics What statistics the parafermions obey is a question that can only be answered fully in the quantum theory of the model. However, we can get some indication of nontrivial statistics through the following semi-classical argument. Define the Green's functions of the quantum theory through the usual functional integral. The parafermions can be defined covariantly on a general surface by

$$
\psi_{\mu}=\frac{i k}{\pi} e^{-i x}\left(g^{-1} D_{\mu} g-\epsilon_{\mu}{ }^{*} D_{\nu} g^{-1}\right) e^{i x}
$$

where $\chi(x)=\int_{C} d y^{\mu} \cdot A_{\mu}(y)$ and $D_{\mu}=\partial_{\mu}-i\left[A_{\mu}\right.$. It is important to realize that in cornputing Green's functions, $A_{\mu}$ should be treated as an independent field to be integrated. In particular, $A_{\mu}$ is no longer a pure gauge, since eq.(2.9) will now have source terms at the location of various fields. Furthermore, since $A_{\mu}$ is not pure gauge, the field $\chi$ and therefore $\dot{\psi}_{\mu}$ explicitly depend on the path $C$. We may think of the path $C$ as a branch cut in space and the resulting Green's function as non-single valued, again reflecting the parafermionic nature of $\psi_{\mu}$. It is possible to investigate this more quantitatively as follow's: Next, consider a field $u$ with chiral charge, i.e., a local field that transforms non-trivially under chiral transformations. Specifically, we can take $u$ to be $g$ transforming as described in section IV. Next, consider the Green's function $G=\left\langle\psi_{\mu}(x) u(y)>\right.$. More generally, $G$ could depend also on other fields, but since they play no role in the tollowing argument, we have suppressed them. Fix $x$ and imagine moving $y$ adjabatically around a closed path circling $x$. Were it not for the branch cut $C, G$ would return to its original value at the end of this adiabatic transport. However, because of the presence of the cut, there is a discontinuous jump in the phase of $G$ as the cut is crossed, and so $G$ acquires a phase upon returning to its original location. To compute this jump in the phase, we need the Ward identity resulting from invariance under these chiral transformations. Consider the Greens function

$$
G\left(x_{1} \ldots x_{n}\right)=\left\langle u\left(x_{1}\right) \ldots u\left(x_{n}\right)\right\rangle .
$$


Now in the functional integral defining this green's function make the finite local chiral transformaijons $g(x) \rightarrow H_{c}(x) g(x) H_{c}(a)$ where $H_{c}=\exp \left(-\frac{i}{2} \sum_{\mathrm{a}} \theta_{\mathrm{a}}(x) h_{\mathrm{a}}\right)$. The functional integral is invariant under this change of variables (assuming the absence of anomalies). Under this the $u$ transform a

$$
\mu(x) \rightarrow \exp \left(i \sum \alpha_{a} \theta_{a}(x)\right) u(x)
$$

The change in the action is given by eq.(5.3) with infinitesimal $\mathrm{d} \theta_{a}$ 's replaced by the finite $\theta$ 's above. Thus we have the following Ward identity

$$
\begin{gathered}
\left\langle\exp \left(\frac{i k}{\pi} \int d^{2} y \sum_{a} \theta_{a}(y) F_{a}(y)\right) u\left(x_{1}\right) \ldots . u\left(x_{n}\right)\right\rangle \exp \left(i \sum_{\ell=1}^{n} \alpha_{a} \theta_{a}\left(x_{l}\right)\right) \\
=\left\langle u\left(x_{1}\right) \ldots u\left(x_{n}\right)\right\rangle
\end{gathered}
$$

Now we are readj to compute the jump in the phase of $G$ of eq.(7.3) as one the coordinates crosses the cut $C$. This crossing can be thought of as a distortion of $C$ by a small area $\Sigma$ and under such a change in $C$ the factor $x$ in the definition of $\psi_{\mu}$ changes by (see eq.(5.6)),

$$
\delta x=\int_{\Sigma} d^{2} y F(y)
$$

and $\psi_{\mu}$ changes by

$$
\psi_{\mu}(x) \rightarrow \exp \left(-\int_{\Sigma} d^{2} y F(y)\right) \psi_{\mu}(x) \exp \left(\int_{\Sigma} d^{2} y F(y)\right) .
$$

So the expectation value of $\left\langle\psi_{\mu}(x) u(y)>\right.$ now acquires extra factors. Let us work out the case of $S U(2) / I(1)$ in detail. In this case, eq.(7.7) becomes (suppressing Lorentz indices)

$$
\psi_{ \pm}-\psi_{ \pm} \exp \left( \pm i \int_{\Sigma} d^{2} y F_{3}(y)\right)
$$

where we recall both $\psi_{ \pm}$and $A_{ \pm}$are in the adjoint representation ( \pm and 3 are Lie algebra indicies not Loreatz indices). Thus we learn that under this transport

$$
\left\langle\psi_{ \pm}(x) u(y)\right\rangle \rightarrow\left(\psi_{ \pm}(x\rangle \exp \left( \pm i \int_{\Sigma} d^{2} z F_{3}(z)\right) u(y)\right)
$$


where $\Sigma$ is a small area which includes the point $y$. Now for $u$ take the combination $u=90+i g_{3}$. Then from the transformation $g \rightarrow H_{c} g H_{c}$ we find

$$
g_{0}+i g_{3} \rightarrow\left(g_{0}+i g_{3}\right) \exp \left(-i \theta_{3}\right)
$$

so $\alpha_{3}=-1$ in eq. (7.4). Now we evaluate eq.(7.9), taking $\theta_{3}(x)= \pm \frac{\pi}{k}$ inside $y$ inside $\Sigma$ and $\theta_{3}=0$ outside $\sum$. This gives

$$
\left\langle\psi_{ \pm}(x) u(y)\right\rangle \rightarrow\left\langle\psi_{ \pm}(x) u(y)\right\rangle \exp \left(\mp \frac{i \pi}{k}\right)
$$

so, $y$ must go around $x$ a full $2 k$ complete times to return to its original value. This agrees with the usual $2 k$-valuedness of parafermions.

2. Bilocality Let us return briefly to the discussion of the $\psi$ 's bilocality and behavior under gauge transformations. If we allow gauge transiormations at $x_{0}$, the base point of integration used in defining $x$ (see eq.(5.6)), then

$$
\partial_{+} \psi=0
$$

is no longer quite correct, for to derive it we needed the step

$$
\partial_{+} \int_{x}^{x} d y A_{-}(y)=\int^{x} d y \partial_{y} A_{+}(y)=A_{+}(y)
$$

making use of $F=\partial_{+} A_{-}-\partial_{-} A_{+}=0$. However, there is a contribution to eq.(6.13). the lower limit, which we neglected. This unpleasantness can be avoided by considering a product of a cluster of parafermionic fields whose chiral charges add up to zero. The chiral charge is the same as the flux carried by the integral in eq. (7.13) and since these fluxes add up to zero, the end point contributions from the common end point $x_{0}$ also cancel. This suggest that in the quantum theory of parafermions, one should only consider neutral Green's functions, i.e. Green's functions with total charge zero. Alternatively, one should introduce a "background" charge at $\pm_{0}$ to balance the total charge. With this proviso, the results of the previous sections remain unaltered. Bowever, notice that the abelian nature 
of the conserved charges is an important restriction in reaching this conclusion. We hope to consider the non-abelian extension of these ideas in a future publication.

3. The $\theta-$ term To the action of Lagranian eq.(2.3) we may add a term

$$
\Delta I=\sum i \Theta_{a} \int d^{2} x F_{a}(x)
$$

where the $\theta_{a}$ are constants. This term is a total divergence and so will not modify the equations of motion in the absence of external sources. If the action is defined on a compact space this term can be integrated to zero if the possible monopole contributions are neglected. Eowever, if a Green's function is computed, this term can no longer be dropped. Consider a field in the Green's function located at point $y_{1}$ which carries chiral charge, i.e., transforms non-trivially under $g-\ddot{H}_{c} g H_{c}$. Then the currents generated by these charges are

$$
\begin{aligned}
\mathcal{J}_{\mu}^{a} & =\frac{k}{\pi} \epsilon_{\mu}{ }^{\nu} A_{\nu} a \text { with } \\
\partial^{\mu} \mathcal{J}_{\mu}^{a} & =\frac{k}{\pi} F^{a}=0
\end{aligned}
$$

in the absences of sources. However, in the presence of the chiral field located at $y$, the eq.(7.14) has a source term on the right hand side:

$$
\frac{k}{\pi} F^{a}(x)=\partial^{\mu} \mathcal{J}_{\mu}^{a}(x)=\text { const. } \cdot \delta^{2}(x-y) .
$$

The integral in eq.(7.13) can then be converted into a path integral around the point $y$ (where the source is located),

$$
\int d^{2} x F_{0}(x) \rightarrow \int_{C} d x^{\mu} A_{\mu}^{a}(x)
$$

where $C$ surrounds $y$. The right hand side of eq.(7.16) can then be thought of as (partially) screening the charge located at $y$. This is analogous to the screening charges of the FeigenFuchs construction, so it is natural to conjecture that there is a connection. Bowever, the Feigen-Fuchs charges are quantized, and it is clear that a treatment that goes beyond the classical approach presented here is needer. 


\section{Conclusion}

Starting with the gauged Wess-Zumino-Witten model, we have used classical ideas to motivate the canonical transformations necesary to recover the conformal coset models. In essence this gives a "top-down" approach to understanding the rather ad-hoc Feigent-Fuchs construction. Essential to the success of this program, the Poisson brackets of the classical parafermions in the gauged WZW model have been computed and yield the expected results.

This work is being extended in several ways, the most interesting being the nonabelian coset ( $h$ is chosen nonabelian.) Much progress has been made on this approach with the hope that it will lead to nonabelian Feigen-Fuchs constructions. 


\section{Acknowledgments}

We would like to thank O. Alvarez, R. Littlejohn, S. Hotes, E. Kiritsis and D. Smit for their comments and interest in this work. This work was supported in part by the Director, Office of Energy Research, Office of Eigh Energy and Nuclear Physics, Division of High Energy Physics of the U.S. Department of Energy under Contract DE-AC03-76SF00098 and in part by the National Science Foundation under grant PHY85-15857

One of us (mc) was supported by a National Science Foundation Fellowship and a Department of Education Grant. 


\section{Appendix}

In this appendix we provide some details about the computation of the Poisson bracket of the classical parafermions eq.(5.16). Recall that in terms of $f$ given in eq.(5.7), $\psi$, the classical parafermion is given by;

$$
\psi=\frac{i k}{\pi} \partial_{-} f f^{-1}
$$

Recall also that $f$ is a gauge invariant quantity. The following calculation detailed in this appendix is rather involved. Although the result eq.(5.16) is simple we have found no equally simple way of deriving it. We now describe a tedious brute force calculation. First, in computing Poisson brackets of two $\psi$ 's it is convenient to contract all of $\psi$ 's indices rather than explicitly displaying them. So, as is done in ref.[7] for the ungauged WZW model, let $P\left(x_{-}\right), Q\left(z_{-}\right)$be arbitrary functions with coset space indices and form $\psi_{P}$ and $\psi_{Q}$ given, for example, by

$$
\psi_{P}=\int d x_{-} \operatorname{Tr}\left(P\left(x_{-}\right) \psi\left(x_{-}\right)\right)
$$

Recalling that $d \psi=\frac{i k}{\pi} f \partial_{-}\left(f^{-1} d f\right) f^{-1}$ we have that

$$
\begin{aligned}
\psi_{P} & =\frac{-i k}{\pi} \int \operatorname{Tr}\left(f^{-1} \mathbf{d} f \partial_{-}\left(f^{-1} P f\right)\right) \\
& =-\int \operatorname{Tr}\left(\mathbf{R} \partial_{-}\left(f^{-1} P f\right)+\frac{i k}{\pi} \mathbf{H} \partial_{-}\left(f^{-1} P f\right)+\mathbf{H}[\psi, P]\right)
\end{aligned}
$$

in which $\psi_{P}$ stands for $d \psi_{P}$ viewed as a form and where the definition $\mathbf{R}=f^{-1}(\mathrm{~d} f-i[\mathbf{H}, f])$ is used. Consider now forming the Poisson bracket $\left\{\psi_{P}, \psi_{Q}\right\}$. Using the form $\psi_{P}$ above, $\left\{\psi_{P}, \psi_{Q}\right\}$ will involve several terms. We parse these as follows;

$$
\begin{aligned}
\left\{\psi_{P}, \psi_{Q}\right\}= & B+\mathcal{C} \text { where } \\
\mathcal{B}= & \left\{\int \operatorname{Tr}\left(\mathbf{R} \partial_{-}\left(f^{-1} P f\right)\right), \int \operatorname{Tr}\left(\mathbf{R} \partial_{-}\left(f^{-1} Q f\right)\right)\right\} \\
\mathcal{C}= & \left\{\int \operatorname{Tr}\left(\operatorname{R} \partial_{-}\left(f^{-1} P f\right)+\frac{1}{2} \mathbf{H}\left(\frac{i k}{\pi} \partial_{-}\left(f^{-1} P f\right)+[\psi, P]\right)\right),\right. \\
& \left.\int \operatorname{Tr}\left(\mathbf{H}\left(\frac{i k}{\pi} \partial_{-}\left(f^{-1} Q f\right)+[\psi, Q]\right)\right)\right\}-(P=Q)
\end{aligned}
$$


Now using eq.(5.14) one may simplify the expression for $B$

$$
\begin{aligned}
\mathcal{B} & =\frac{k}{\pi} \int \operatorname{Tr}\left(f^{-1} Q f \partial_{-}\left(f^{-1} P f\right)\right) \\
& -\frac{k}{2 \pi} \int \sum_{a} \operatorname{Tr}\left(h_{a} f^{-1} Q f\right) \operatorname{Tr}\left(h_{a} \partial_{-}\left(f^{-1} P f\right)\right) \\
& =\frac{k}{\pi} \int \operatorname{Tr}\left(Q \partial_{-} P\right)+i \int \operatorname{Tr}([P, Q] \psi) . \\
& -\frac{k}{2 \pi} \int \sum_{a} \operatorname{Tr}\left(h_{a} f^{-1} Q f\right) \operatorname{Tr}\left(h_{a} \partial_{-}\left(f^{-1} P f\right)\right)
\end{aligned}
$$

where the term involving $\left\{\operatorname{Tr}\left(h_{a} f^{-1} Q f\right) \operatorname{Tr}\left(h_{a} \partial_{-}\left(f^{-1} P f\right)\right)\right.$ arises in $B$ because the traces are over all the generators whereas in eq. (5.14) the R's poist only into the coset (the sum $\sum_{a}$ is over generators of $\mathcal{H}$ ).

To simplify $C$ we must compute Poisson brackets of $\mathbf{H}$ with $\mathbf{H}$ and $\mathbf{R}$. Since $\boldsymbol{H}=$ $\int^{x} d x_{-} \frac{1}{2} \epsilon\left(x-x_{-}\right) F\left(x_{-}\right)$and $H$, as a form, is defined below eq.(5.12) in the text, eq.(5.4) implies eq. (5.15),

$$
\begin{aligned}
& \left\{\mathrm{d} \theta^{a}(x), \mathrm{H}^{b}(y)\right\}=\frac{-\pi}{2 k} \delta^{a b} \epsilon(x-y) \\
& \left\{\mathrm{d} \rho^{c}(x), \mathrm{H}^{b}(y)\right\}=0
\end{aligned}
$$

where, again, the index $\ell$ runs only over generators in the set $q$ / $\boldsymbol{t}$. Since $H$ has non-zero Poisson brackets with $\theta^{a}$ co-ordinates only, then in order to compute the Poisson bracket between $\mathbf{H}$ and $\mathbf{R}$ we need only isolate the $\mathbf{d} \theta$ part of $\mathbf{R}$. Now recalling the definition $\mathbf{R}=f^{-1}(\mathrm{~d} f-i[\mathrm{H}, \lambda)$ we have,

$$
\begin{aligned}
& \operatorname{Tr}\left(\operatorname{R} \partial_{-}\left(f^{-1} P f\right)+\frac{1}{2} \mathbf{H}\left(\frac{i k}{\pi} \partial_{-}\left(f^{-1} P f\right)+[\psi, P]\right)\right) \\
& \quad=\operatorname{Tr}\left(f^{-1} \mathrm{~d} f \partial_{-}\left(f^{-1} P f\right)-\frac{1}{2} \mathrm{H}\left(\frac{i k}{\pi} \partial_{-}\left(f^{-1} P f\right)+[\psi, P)\right)\right)
\end{aligned}
$$

and now using eq. (5.10) we may rewrite this term as,

$$
\operatorname{Tr}\left(\pi \partial_{-}\left(f^{-1} P f\right)+\frac{1}{2} \mathrm{H}\left(\frac{i \hbar}{\pi} \partial_{-}\left(f^{-1} P f\right)+[\psi, P]\right)\right)
$$




$$
\begin{aligned}
& =\operatorname{Tr}\left(\sum_{a} \mathrm{~d} \theta^{\infty}\left[\frac{i}{2}[\psi, P] h_{a}+\frac{k}{2 \pi} h_{a} \partial_{-}\left(f^{-1} P f\right)\right]\right. \\
& \left.+\frac{(x+\mathbf{A})}{2}\left[\frac{i k}{\pi} \partial_{-}\left(f^{-1} P f\right)+[\psi, P]\right]+\ldots\right)
\end{aligned}
$$

where ... means terms involving $\mathrm{d} \rho^{\prime}$ that won't contribute to the Poisson bracket of $\mathcal{C}$ in eq.(A.4), Note that we have used the fact that $\mathbf{H}=\boldsymbol{x}-\mathbf{A}$. Putting this into $\mathcal{C}$ one finds that the terms involving $\{\boldsymbol{x}+\mathbf{A}, \boldsymbol{x}-\mathbf{A}\}$ vanish identically by antisymmetry in $P$ and $Q$. Then $\mathcal{C}$ simplifies to

$$
\begin{aligned}
C= & \left\{\int \operatorname { T r } \left(\sum \mathrm{d} \theta^{a}\left(\frac{i}{2}[\psi, P] h_{a}+\frac{k}{2 \pi} h_{a} \partial_{-}\left(f^{-1} P f\right)\right),\right.\right. \\
& \left.\int \operatorname{Tr}\left(H\left(\frac{i k}{\pi} \partial_{-}\left(f^{-1} Q f\right)+[\psi, Q]\right)\right)\right\}-(P \leftrightarrow Q) \\
= & \frac{\pi}{4 k} \int d x d y \epsilon(x-y) \operatorname{Tr}\left(\left[h_{a}, P\right] \psi\right)(x) \operatorname{Tr}\left(\left[h_{a}, Q\right] \psi\right)(y) \\
+ & \frac{k}{2 \pi} \int \sum_{a} \operatorname{Tr}\left(h_{a} f^{-1} Q f\right) \operatorname{Tr}\left(h_{a} \partial_{-}\left(f^{-1} P f\right)\right)
\end{aligned}
$$

where use has been made of eq.(A.6). Now combining $\mathcal{B}$ and $C$ we find the final result

$$
\begin{aligned}
\left\{\psi_{P}, \psi_{Q}\right\} & =\frac{2 k}{\pi} \int \operatorname{Tr}\left(Q \partial_{-} P\right)+2 i \int \operatorname{Tr}([P, Q] \psi) \\
& +\frac{\pi}{2 k} \iint d x d y \epsilon(x-y) \sum_{a} \operatorname{Tr}\left(\left[h_{a}, P\right] \psi\right)(x) \operatorname{Tr}\left(\left[h_{a}, Q\right] \psi\right)(y)
\end{aligned}
$$

This resu:. is of a rather simple form considering the derivation. Note that all the terms of eq.(A.10) are relevant and that the first one becomes the central term is the algebra of eq. (5.16). 


\section{References}

1. H, Sugawara, Phys. Rev. 170, 1659 (1968).

2. P. Goddard, A. Kent and D. Olive, Phys. Lett. B152, 88 (1985).

3. D. Friedan, Z. Qiu and S. Shenker, Phys. Rev. Lett. 52, 1575 (1984).

4. A. Belavin, A.M. Polyakov and A.B. Zamolodchikow Nucl. Phys. B241, 33 (1984).

5. K. Bardakci and M.B. Halpern Nucl. Phys. B73, 295 (1974).

6. M.B. Halpers, et al., LBL-27623 to appear in Int. J. Mod. Phys. A; M.B. Halpern and N.A. Obers LBL-28245; A. Yu Morozov, M.A. Shifman and A.V. Turbiner, Int. J. Mod. Phys. A5. 2953 (1990).

7. E. Witten, Comm. Math. Phys. 92, 455 (1984).

8. A.M. Polyakov and P.B. Wiegmann, Phys. Lett. B131, 121 (1983); Phys. Lett. B141, 223 (1984).

9. D. Kiarabali and H. Schnitzer, Nucl. Phys. B328, 649 (1990)

10. D. Bernard and G. Feider ETH-TH/89-26.

11. K. Bardakci, E. Rabinovici and B. Säring Nucl. Phys. B299, 151 (1988).

12. A.B. Zamolodchikov and V.A. Fateev, Sov. Phys. J.E.T.P. 62, 215 (1985).

13. M. Wakimoto, Comm. Moth. Phys. 104, 605 (1986).

14. D. Nemeschansky, Phys. Lett. B224, 121 (1989), USC-89/012

15. A. Bilal, CERN-TH 5370/89.

16. D. Frierlan, Ann. Phys. (NY) 163, 318 (1985). 
17. E. Braten, T. Curtright and C. Zachos Nucl. Phys. B260, 630 (1985).

18. V.S. Dotsenko and V.A. Fateev, Nucl. Phys. B240, 312 (1984).

19. I. Antoniadis and C. Bachas, Nucl. Phys. B278, 343 (1986); E. Tomboulis, UCLA/86/TEP/3। Phys. Lett. B188, 165 (1987). The idea of gauging sigma models in this way goes back to S. Coleman. J. Wess and B. Zumino, Phys. Rev. 177, 2239 (1969); C. Callan. et. al., Phys. Rev. 177, 2247 (1969).

20. P. Bowcock, Niucl. Phys. B316, 80 (1989) in this paper the Poisson brackets of various currents were computed but the Poisson brackets of non-local quantitates, such as the classical parafermions, was not considered.

- 21.. A. Gerasimov, et. al.,Int. J. Mod. Phys. A5, 2495 (1990), Nucl. Phys. B328. 664 (1989); for other modern references on the bosonization of WZW Models refer to $\mathrm{k}$. Gawedski, an IHES preprint and G. Dunne, I. Halliday and P. Surayni, Nucl. Phys. B325, 526 (1989). 


\section{Contributions and Interests}

This author was involved with this work from it's inception. The motivation was again provided by Prof. K. Bardakci (thesis advisor) and this author's contributions include checking the form of the lagrangian (eq.(2.1) and eq.(2.3)), identification of the currents in eq. (2.4) and checking the identity eq.(2.8). This author suggested trying to use a pa ular technique for computing Poisson brackets (based on a reference) that turned out to be useful and the calculation using this technique became the major part of the paper. Among other contributions this author derived the eq.(4.8) and thereby provided a simple derivation of eq.(4.13) (as detajled in eq.(4.9) through eq.(4.13)), used eq.(4.8) to derive eq.(5.3) (and therefore eq.(5.4) and eq.(5.14)) and checked the rest of the computation of eq.(5.16).

In section II this author started with the eq.(2.3) and checked that after gauge fixing that one has eq. (6.1) and then proceeded to analyze the classical equations of motion for the lagrangian eq.(6.1) ultimately finding an interesting geometrical solution. These solitonic solutions were shown in eq.(6.4) and eq.(6.5) to correspond to the classical parafermions.

Of particular interest for this author is the form of the Poisson bracket eq.(5.16) and whether it is possible to write the general result for a non-abelian coset in a similar fashion and to understand better the relation between the local chiral coset lagrangians described in section VI and the exitations (or even the conformal invariance) of the underlying conformal field theory. In the next paper it will be shown that the Poisson bracket of a non-abelian coset model may be written in a form similar to that of eq.(5.16) but with some interesting suprises. As for the other point, this author has written down the chiral coset lagrangian for a non-abelian coset (that of $S U(2)_{n} \times S U(2)_{m} / S U(2)_{n+m}$ ) and has done a preliminary search for the solitons (non-trivial solutions to the equations of motion) of this theory finding anong other things that the regularity of the solutions requires that $m / n$ be a rational number (this is true quantum mechanically and so it being a requirernent classically is a 
sign that these solitons may be semiclassical configurations. 


\title{
Parafermions from non-Abelian Coset Models
}

\author{
K. Bardakci \\ M. Crescimanno \\ and \\ S. Hotes \\ Department of Physics \\ University of California \\ Berkeley, Califorria 94720 \\ and \\ Laurence Berkeley Laboratory
}

in Nuclear Physics B, Vol. 349 (1991), pg. 439 


\section{Introduction}

Cocet modelo $1,2.3$ are among some of the most interesting examples of two dimensional conformal theories ${ }^{4,5}$. The primary fields of these models and their various properties are under intensive study at the present time 6 . Most of the approaches used so far are algebraic in nature; however, it is well known that an alternative method ${ }^{7}$ that starts with the gauged WZW lagrangian is also available ${ }^{8}$. In this latter approach, the question of how to fix the gauge immediately arises. A popular choice is the axial gauge ${ }^{9}$, which leads to

a description in terms of non-interacting WZW models ${ }^{10-14}$, some of which correspond to ghosts. The BRST intariance plays a crucial role in establishing unitarity $14,15,16$; a situation that is quite standard in gauge theories in general when an unphysical gauge choice is made. An alternative possibility is the unitary (physical) gauge ${ }^{17}$, which was used to investigate abelian coset models in an earlier paper ${ }^{14}$. It was shown in that paper that coset models can be reformulated in terms of fields which are chirally conserved, gauge in"ariant and which satisfy a simple closed PB (Poisson bracket) algebra. It was then natural to identify these fields with parafermions ${ }^{19}$, whicl. are known to be the primary fields of the coset models 20 . The PB algebra was shown to lead easily to the free-field realizations $21,22,23$ of the parafermionic fields.

In ref, [18] the factor group of the coset was taken to be abelian, although it was pointed out that most the results easily generalize to the non-abelian case. Our main goal in this paper is to carry out this generalization. We shall see that, although many of the results carry over easily, there are some important differences as well. Our approach is the same semi-classical approach used in ref.[18]; we start with the gauged WZW lagrangian, define (classically) the parafermion fields, compute their PB's, use these results to give a simple construction for the parafermions, and finally quantize the model.

The paper is arranged as follows: In section II, we review briefly the gauged WZW 
modal, the construction of the parafermionic fields and the calculation of their PB's. The latter calculation is somewhat involved and it was presented in full detail in the abelian case in ref.[18]. Here we do not repeat it but instead we focus on the new complications that arise in the non-abelian case. The basic problem is the following: the parafermionic fields are constructed by attaching a Wilson line to the currents of the WZW model to make them gauge invariant. and although these fields are locally gauge invariant, they transform non-trivially under the group that operates at the end point of the Wilson line. Parafermions therefore carry a (globally) conserved quantum number (color), associated with a fixed end point, which we shall call the base point. The completely gauge invariant quantities are constructed out of parafermions by forming "color" singlet combinations, and they are the true physical tariables of the theory. They are also multilocal, since they are functions of several - rdinates. For this reason, in calculating PB's of vajous quantities it is usually easier - $\quad$ t'k with parafermions rather than 'ly with gauge invariant quantities. Unfortunately, as we shall show, the PB's of parza . Nons close into themselves only in the abelian case; the PB's of non-abelian parafermions receive additional complicated contributions from the base point. We shall argue that these base point contributions are gauge artifacts which cancel in calculating the PB's among gauge invariant (color singlet) variables, and this naturally leads us to define "generalized" PB's where the base point terms are dropped. A price is paid in doing this; the generalized brackets no longer satisfy the Jacobi identity and therefore generate a non-associative algebra. However, we prove in appendix A that the color singlet sector of this algebra is associative, which is to be expected since this sector is gauge inverialt and base point independent. Therefore, in one sense, the non-associative algebra is purely a calculational tool; on the ncher band, it is possible to view it as parafermions having non-associative statistics. Of course, since parafermions are by construction confined in this model, this abnormal statistics is not directly observable. It is amusing to note that a special non-ascociative statistics bas already been proposed as 
an alterative description ci quark cofinement ${ }^{24}$.

In the next two sections of the paper (sections III and IV), we describe an explicit realization of the parafermion fields which we call the free current construction. This can be viewed as a natural generalization of the well-known free field realizations of the abelian coset models $25,20,27$ except in this case free fields are replaced by currents that satisfy the affine algebra ${ }^{28,2}$ generated by the original Lie group. The basic idea is simple; the Wilson lines attached to the parafermions are replar.ad by the corresponding path ordered exponentials of the free currents. We show in section III that, classically, this costruction rep-oduces all the properties of the parafermion fields; in particular, again neglecting the base point contributions, the PB's derived in section II are recovered.

Our treatment thus far has been classical; however the free current construction suggests a natural method of quantization. The basic idea, pursued in section $\Gamma V$, is to replace the classical free currents that satisfy the aftine PB algebra by operators that satisfy the corresponding commutator algebra. This process inevitably leads to divergences which have to be sliminated by renormalization. We conjecture that all color singlet amplitudes are rendered finite by multiplicative renormalization, and we verify our conjecture in lowest order perturbation. Once the operator construction is renormalized, it should be possible to extract all cf the primary fields of the model through the short distance expansion of the color singlet operators; the gauge invariant, multilocal operators constructed out of free currents therefore "package" the primary fields of the coset model. At the cost of having to deal with non-local operators, this approach bas the advantage of avoiding complications of gauge dependent methods, such as ghost fields and screening charges, common in FeiginFuchs type constructions $2 \%$. The last section summarizes our conclusions and suggests porsible extensions. 


\section{Reviow of the Gauged WZW Model}

We start with the Wess-Zumino-Witten model in light-cone coordinates $\left(x_{ \pm}=x_{0} \pm\right.$ $\left.x_{1} ; \partial \pm=1 / 2\left(\partial_{0} \mp \partial_{1}\right)\right)$, where $g$ takes values in some Lie group $G$.

$$
I W z W=\frac{k}{4 \pi} \int \mathrm{d}^{2} x \operatorname{tr}\left(\partial_{+} g^{-1} \partial_{-} g\right)+\frac{k}{24 \pi} \int \mathrm{d}^{3} x \varepsilon^{i j k} \operatorname{tr}\left\{g^{-1} \partial_{i} g g^{-1} \partial_{j} g g^{-1} \partial_{k} g\right\}
$$

Letting $H$ be some subgroup of $G$, and $\mathbf{h}$ the Lie algebra of $B$, we define :

$$
I=I \mathrm{WZW}+\frac{k}{2 \pi} \int \mathrm{d}^{2} x \operatorname{tr}\left\{i A_{+} \partial_{-} g g^{-1}-i A_{-} g^{-1} \partial_{+} g+A_{+} g A_{-} g^{-1}-A_{+} A_{-}\right\}
$$

where $A_{ \pm}$takes ralues in $h$ The gauge fields $A_{ \pm}$act as Lagrange multipliers that remove the components 'f the conserved currents lying in the subgroup $B$. We now have the currents,

$$
J_{+}=\frac{i k}{\pi} g^{-1} D_{+} g ; \quad J_{-}=-\frac{i k}{\pi}\left(D_{-} g\right) g^{-1}
$$

where $D_{ \pm} g=\partial_{ \pm} g-i\left[A_{ \pm}, g\right]$. Letting $F=\partial_{+} A_{-}-\partial_{-} A_{+}-i\left[A_{+}, A_{-}\right]$we have the following equations of motion

$$
\begin{gathered}
\operatorname{tr}\left(h_{\mathrm{a}} J_{ \pm}\right)=0 ; h_{\mathrm{a}} \in \mathrm{h} \\
D_{+} J_{-}=0=D_{-} J_{+} \\
F=0 .
\end{gathered}
$$

As Lagrange multipliers the gauge fields $A_{ \pm}$can be solved explicitly in terms of $g=g\left(x_{ \pm}\right)$.

In order to simplify the quantization procedure, we may perform a convenient canonical transformation on the classical fieldy such that the resulting pbyjical degrees of freedom are the paratermions $\psi$. We expect $\psi$ to have the following properties :

1. Corat-valued: We have used the gauge field to project out the subgroup components, leaving only coset-valued degrees of freedom, i.e. we expect:

$$
\operatorname{tr}\left(h_{a} \psi\right)=0 \quad W^{\star} \in \mathbf{b}
$$


2. Geuge Invariant: We ask that the coupling introduced in eq.(2.2) render the action gauge invariont under :

$$
g \rightarrow B^{-1} g B, \quad A_{ \pm} \rightarrow B^{-1} A_{ \pm} B+i B^{-1} \partial_{ \pm} B ; \quad B \in H
$$

3. Chirally Conserved: The aniicipated conformal structure of the underlying coset model suggests :

$$
\partial_{\mu} \psi^{\mu}=0, \quad \partial_{\mu}\left(\varepsilon^{\mu \nu} \psi_{\nu}\right)=0
$$

Wits these properties in mina, we define

$$
\psi_{\mu}\left(x, x_{0}\right)=U^{-1}\left(x, x_{0}\right) J_{\mu}(x) U\left(x, x_{0}\right)
$$

where $J$ is the current defined in eq. (2.3) and $U$ is the Wilson line defined by

$$
U\left(x, x_{0}\right)=P_{C} \exp \left(i \int_{x_{0}}^{x} d x_{\mu}^{\prime} A^{\mu}\left(x^{\prime}\right)\right)
$$

$P_{c}$ represents "pach ordering" the exponential slong a curve $C$ which connents the "base point ${ }^{n} x_{0}$ to $x$. The Wilson line $U$ satisfies the important semigroup properties

$$
U\left(x_{1}, x_{3}\right) U\left(x_{3}, x_{3}\right)=U\left(x_{1}, x_{3}\right) ; \quad U^{-1}\left(x_{1}, x_{2}\right)=U\left(x_{2}, x_{1}\right)
$$

Sirce $F=\mathbf{0}$ classically, $U$, and consequently $\psi$, do not depend on the path $\mathbf{C}$, but only on the endpoints. Fixing the base point $x_{0}$ we view $\psi$ as 3 function of a single variable x. Defining $g$ as the Lie algebra of $G, J(x) \in \varepsilon-h$ and therefore $\psi$, which is just $J(x)$ conjugated by an element of $\boldsymbol{H}$, is also a member of the coset $g-h$. Ifnoring base point contributions (which will be considered shortly), $\psi$ is locally gauge invariant and chiral!y conserved. These properties follow from the equations of motion eq.(2.4), nd the Wilson line identity

$$
\dot{x}^{\mu}\left(\partial_{\mu}^{x}-i A_{\mu}(x)\right) U\left(x, x_{0}\right)=n_{i} \quad \dot{x}^{\mu} \text { is tangent to } C \text { at } x
$$


To construct Poisson brackets, we will identify $x_{+}$with time, and $x_{-}$with space, and focus on the $\psi_{-}$component of $\psi$, which satisfies

$$
\partial_{+} \psi_{-}=0 \Rightarrow \psi_{-}=\psi_{-}\left(x_{-}\right)
$$

For the computation of the Poisson bracket it is advantageous to replace $U$ defined along the $x_{+}$axis with $U$ defined along the $x_{-}$axis; using the equation of motion $F=0$. In the sequel, when no space-time index appears, a space index $(-)$ is implied. We have,

$$
\begin{gathered}
U\left(x, x_{0}\right)=P \exp \left(i \int_{x_{0}}^{x} d x^{\prime} A_{-}\left(x^{\prime}\right)\right) \\
\longleftarrow \equiv \psi_{-} \equiv-U^{-1}\left(x, x_{0}\right) J_{-}(x) U\left(x, x_{0}\right)
\end{gathered}
$$

The integration being calried out a: fixed time (fixed $z_{+}$).

For the physical obsertables, the above mentioned base point contributions should vanish. A complete set of such variables constructed from the local parafermions is given by" the following set of "multilocal" observables

$$
\begin{gathered}
O\left(x_{1}, x_{2}, \cdots x_{n}\right)=\operatorname{tr}\left\{\psi\left(x_{1}\right) \psi\left(x_{2}\right) \cdots \psi\left(x_{n}\right)\right\} \\
=(-1)^{n} \operatorname{tr}\left\{J\left(x_{1}\right) U\left(x_{1}, x_{2}\right) J\left(x_{2}\right) \cdots J\left(x_{n}\right) U\left(x_{n}, x_{1}\right)\right\} .
\end{gathered}
$$

"Multilocal" is used in the following sense: The local parafermion is equipped with a nonabelian tail, analogous to the Dirac tail of the electromagnetic monopole. When we "tie" these tails together in a gauge invariant manner, a shown above, we remove this non-local dependence. If we were to reinstate the full two-dimensional freedom of these objects. we would find that two non-overlapping observables $O_{1}$ and $O_{2}$ would satisfy Bose statistics with respect to one-another.

Truly local objects can also be extracted from these observables by letting the space variables approach each other and expanding in the differences $\left(x_{i}-x_{j}\right)$. When we quantice. 
this procedure will give us the operator product expansion, the coefficients of this expansion being identified as the primary fields. The parafermions are treated as building blocks of the physical observables. We find that in general it is much exsier to work with the parafermions $\psi$, rather than the observables $O_{i}$ directly. 


\section{Poisson Bracket of Non-Abelian Coset Parafermions}

In order to quantize this theory, treating the parafermions as local observables, we need first to compute the classical Poisson brackets of the parafermions. This calculation is somewhat involved, and was given in detail in our first paper concerning abelian coset models. Our aim in this section will not be to repeat this calcuiation, but to pinpoint the major differences between the abelian and non-abelian cases. In the present non-abelian case, complications arise due to base point dependent terms. In retrospect, these cornplicated terms can be dropped at the cost of introducing a non-associative generalized Poisson algebra for the parafermions. When we project onto the "multilocal" observables defined above, the base point dependent terms cancel and associativity of the Poisson algebra is restored. We see then that at this stage, the non-associative algebra may be vieved simply as an aid in simplifying algebraic calculations. We begin by reviewing the computation of Poisson brackets for lagrangians linear in time derivatives. Let

$$
I=\int d t \cdot A_{i}(\phi) \frac{d \phi_{i}}{d t}
$$

The variation of I is given by

$$
\delta I=\int d t \delta \phi^{i} E_{i j} \frac{\mathrm{d} \phi_{j}}{\mathrm{~d} t} ; \quad E_{i j} \equiv \frac{\partial A_{i}}{\partial \phi_{j}}-\frac{\partial A_{j}}{\partial \dot{\phi}_{i}} .
$$

Defining the exact one-form $d \phi^{i}=d \phi^{i}$, we have the following Poisson bracket:

$$
\left\{\mathrm{d} \phi^{i}, \mathrm{~d} \phi^{j}\right\}=\left(E^{-1}\right)^{j i}
$$

In the sequel, $\{$,$\} will refer to the classical Poisson bracket. wheras [,] will represent commu-$ tation in the Lie algebra. We generalize this procedure to include inexact forms. Boldface characters "V" will represent one-forms in the field space, while plain characters " $V^{\text {" }}$ represent the corresponding dynamical variables linear in time derivatives :

$$
V^{a}=V_{i}^{a}(\phi) \mathrm{d} \phi^{i} ; \quad V^{a}=V_{i}^{a}(\phi) \frac{\mathrm{d} \phi^{i}}{\mathrm{dt}}
$$


If the variation takes the form,

$$
\delta I=\int d t V^{i}(\phi) E_{i j}(\phi) W^{j}(\phi),
$$

then we have the result,

$$
\left\{V_{i}, \mathbf{W}_{j}\right\}=\left(E^{-1}\right)^{j i}
$$

If $E$ is not invertible, constraints must be imposed on the dynamical fields. In applying this procedure to the action given in eq. (2.2), invertibility is achieved through gauge fixing. The detailed form of this constraint is not important since we will work only with gauge invariant quantities (up to surface terms at the base point). For example, we replace the group element $g(x)$ by the gauge invariant function $f(x)$,

$$
f(x)=U\left(x_{0}, x\right) g(x) U\left(x, x_{0}\right)
$$

and thus we have,

$$
\psi=\frac{i k}{\pi}\left(\partial_{-} f\right) f^{-1}
$$

We want to express $\delta I$ in the form of eq.(3.5). To do this we introduce the following gauge invariant quantity

$$
H(x)=\int_{x_{0}}^{x} \mathrm{~d} x^{\prime} U\left(x_{0}, x^{\prime}\right) F\left(x^{\prime}\right) U\left(x^{\prime}, x_{0}\right)
$$

and its corresponding form

$$
\mathbf{H}(x) \equiv \int_{x_{0}}^{x} \mathrm{dx} U\left(x_{0}, x^{\prime}\right) \mathbf{F}\left(x^{\prime}\right) U\left(x^{\prime}, x_{0}\right)
$$

The form $F$ is derived from $F$ by replacing $\partial_{-g}$ by dg as explained earlier. We have

$$
\delta I=\frac{k}{2 \pi} \int \mathrm{d}^{2} x \operatorname{tr}\left\{f^{-1}(\mathrm{~d} f+i[\mathrm{H}, f]) \partial_{-}\left(f^{-1}\left(\partial_{+} f+i[H, f]\right)\right)\right\}
$$

The symbol $n \sigma^{n}$ signifies the variation of the action under a variation $\mathrm{d} g$ of the field variable. This notation is employed in an effort to conform to the mathematical literature. Using eq. (3.6), we can immediately read of the PB's (Poisson brackets)

$$
\left\{\mathbf{R}^{\ell}(x), \mathbf{R}^{m}(x)\right\}=-\frac{\pi}{2 k} \delta^{\ell m} \varepsilon(x-y)
$$




$$
\text { with } \mathbf{R} \equiv f^{-1}(\mathrm{~d} f+i[\mathrm{H}, f])=\mathrm{R}^{\ell} \tau^{\ell}
$$

$\tau^{f}$ talking values in the coset $g-h$. The aim is to compute the PB's of $\psi$. We express d is terms of $\mathbf{R}$ and $\mathbf{H}$,

$$
\mathrm{d} \psi=\frac{i k}{\pi} \mathrm{f} \partial_{-}\left(f^{-1} \mathrm{~d} f\right) \mathrm{f}^{-1}=\frac{i k}{\pi} \mathrm{f} \partial_{-}\left(\mathrm{R}-i f^{-1}[\mathrm{H}, f]\right) f^{-1}
$$

In the ungauged WZW action, the term with $H$ is absent, and the PB's of the parafermions can be immediately evaluated using eq.(3.12). However, in the present case, it is also necessary to compute the PB's of $\mathbf{H}$ with both $\mathbf{H}$ and $\mathbf{R}$. Since $\boldsymbol{H}$ is expressed in terms of $U$ and $F$, it is important to observe that there exists a simple set of field variables canonically conjugate to $F$. Consider the variation of $g(x)$ generated by infinitesimal "axial" transformations.

$$
\delta_{\mathcal{A}} g=-\frac{i}{2}\left[h^{2}, g\right]_{+} d \theta^{\circ},
$$

with [.]+ being the anticommutator and $h^{\alpha} \in h$. Under this transformation, the variation of the action has the simple form

$$
\delta I=\frac{k}{\pi} \int \mathrm{d}^{2} x d \theta^{a} F^{a} ; \quad F=F^{a} h^{a} .
$$

From this expression we deduce

$$
\left\{\mathrm{d} \theta^{a}(x), F^{b}(y)\right\}=\frac{\pi}{k} \delta^{a b} \delta(x-y) .
$$

To compute the $\mathrm{PB}$ of an arbitrary form $\mathbf{V}$ with $F$, we isolate the part of $\mathbf{V}$ corresponding to the axial variations given in eq.(3.15),

$$
\mathrm{V}=V_{\mathrm{a}}^{(1)} \mathrm{d} \theta^{a}+V_{l}^{(2)} \mathrm{d} \rho^{\ell}=\mathrm{V}^{\ell} \tau^{l}
$$

where $\rho$ 's are the coordinates which supplement the axial coordinates $\theta$. Then

$$
\left\{V^{a}(z), F^{b}(y)\right\}=\frac{\pi}{k} V_{a}^{(1)}(x) \delta(x-y)
$$


Therefore, it is sufficient to isolate the $d \theta$ components in $\mathbf{R}$ and $\mathbf{F}$ to complete the calculation of the PB's of two parafermions. The final result is best expressed in an index free notation. To that end define:

$$
\psi_{P} \equiv \int d x \operatorname{tr}(P(x) \psi(\mathbf{x})) ; P(x) \in \mathbf{g}-\mathbf{h}
$$

The result is

$$
\begin{gathered}
\left\{\psi_{P}, \psi_{Q}\right\}=\frac{2 k}{\pi} \int d x \operatorname{tr}\left(Q\left(\partial_{-} P\right)\right)+2 i \int d x \operatorname{tr}([P, Q] \psi) \\
+\frac{\pi}{2 k} \int d x d y \in(x-y) \sum_{a} \operatorname{tr}\left(\left[\mathrm{h}_{a}, P(x)\right] \psi(x)\right) \operatorname{tr}\left(\left[\mathrm{h}_{a}, Q(y)\right] \psi(y)\right)
\end{gathered}
$$

the sum being carried out over the subalgebra $h$. This is the result given in ref.[1] for $H$ abelian. It is important to check that the above algebra satisfies the Jacobi identity,

$$
\sum_{\text {oretie }}\left\{\left\{\psi_{P_{1}}, \psi_{P_{2}}\right\}, \psi_{P_{3}}\right\}=0
$$

the sum being taken over cyclic permutations of 1,2 and 3 . This identity is satisfied by eq.(3.21) when $H$ is an abelian subgroup of $G$, but in general is violated for non-abelian $H$ (see appendix $A$ for details). The term responsible for this violation is the third term in eq.(3.21), which is non-local and is responsible for the unusual statistics satisfied by $\dot{\psi}^{18}$. Note that the first two terms are the standard local terms of affine Lie algebras, and clearly satisfy the Jacobi identity.

The Jacobi identity is preserved under canonical transformation, so a mistake has to have been made. The error can be traced back to our deduction of eq.(3.1i) from eq.(3.16). which is valid only if the components of $F$ are linearly independent. That is, only if there is no linear constraint of the form

$$
\sum_{a} F^{a} C_{a}=\text { ? }
$$

where $C_{a}$ depends on $g(x)$ but not on $\delta_{t} g(x)$. Suci relations(s) indicate we are working with a constrained system and the right hand side of eq.(3.17) must be modified to

$$
\left\{d \theta^{a}(x), F^{b}(y)\right\}=\frac{\pi}{k} \delta(x-y) P^{a b}
$$


where $P^{a b}$ is the projection of the unit operator $\delta^{a b}$ in the subspace orthogonal to the constraint(s). The existence of such a constraint can be seen from a purely group theoretical point of view. In addition to eq.(3.15), consider the infinitesimal vector (gauge) transformation

$$
\delta_{V g}=-\frac{i}{2}\left[h^{\circ}, g\right] \mathrm{d} \chi^{a}
$$

The existence of a linear relation between $\delta_{A} g$ and $\delta_{V} g$ would imply that a certain linear combination of axial transformations is equivalent to a gauge transformation, which leaves the action unchanged. Since $d \theta$ is arbitrary, this implies a linear relation on $F$ of the form eq.(3.23). If $h$ is the abelian Cartan subalgebra of $\mathbf{g}$ then no such relation exists, and eq.(3.17) is unchanged. This can be proved trivially by diagonalizing $h$ and writing out such a linear relation in componerits. However, if $\mathbf{h}$ is non-zbelian the situation is quite different. Consider for example the case $G=\mathrm{SU}(2) \times \mathrm{SU}(2)$, ind $B=\mathrm{SU}(2)$ (the diagonal subgroup). If we take $\mathrm{SC}(2)$ in the fundamental representation, we can paramaterjze $\left(g_{1}, g_{2}\right)$ as,

$$
\begin{gathered}
g_{1}=a_{0} I_{1}+i \bar{a} \cdot \partial_{1} ; \quad g_{2}=b_{0} I_{2}+i \vec{b} \cdot \bar{\sigma}_{2} \\
\text { with }\left(a_{0}\right)^{2}+(\vec{a})^{2}=1 ; \quad\left(b_{0}\right)^{2}+(\vec{b})^{2}=1 .
\end{gathered}
$$

Here $\sigma_{i}$ for $i=1,2$ represent the Pauli matrices that generate the two SU(2) factors, and I the corresponding unit matrix. In this notation, we can parameterize the subalgebra $h$ by

$$
h=\overrightarrow{\mathrm{h}} \cdot\left(\vec{\sigma}_{1}+\vec{\sigma}_{2}\right) ; \quad h \in \mathbf{h} .
$$

The desired linear relation is then given by

$$
\begin{gathered}
{\left[g_{1} \otimes g_{2}, \vec{n} \cdot\left(\vec{\sigma}_{1}+\vec{\sigma}_{2}\right)\right]_{+}=\left[g_{1} \otimes g_{2}, \vec{m} \cdot\left(\vec{\sigma}_{1}+\vec{\sigma}_{2}\right)\right]} \\
\overrightarrow{\mathbf{n}}=\frac{\vec{a} \times \vec{b}}{|\vec{a} \times \vec{b}|} ; \quad \vec{m}=\frac{b_{0} \vec{a}-a_{0} \vec{b}}{|\vec{a} \times \vec{b}|} .
\end{gathered}
$$




\section{It follows that}

$$
\begin{gathered}
\sum_{a=1}^{3} n^{a} F^{a}=0 \\
\left\{\mathrm{~d} \theta^{a}(x), F^{b}(y)\right\}=\frac{\pi}{k} \delta(x-y)\left(\delta^{a b}-n^{a} n^{b}\right) .
\end{gathered}
$$

The existence of such linear relations of this type, in many cases, follow from simple counting arguments. For exainple, consider the coset $\mathrm{SU}(5) / \mathrm{SU}(4)$. The dimension of $\mathrm{SU}(5)$ is 24 , while for generic $g$, the rank of $\delta_{A}$ and $\delta_{V}$ are 15 and 12 respectively. Since $15+12>24$, linear relations must exist. It is not true that such degene:acies exist for all non-abelian cosets, however they did exist in most of the cases we studied.

The modification of the PB of $\mathrm{d} \theta$ and $F$ changes the computation of the PB's of the parafermions in a subtle way. This modification tells us that a subset of chiral transformations are gauge transformations, and we are instructed to project out these variations in the PB relations. Such a projection is unnecessary when computing PB's between gauge invariant quantities. For example, both $\mathbf{R}$ and $\mathbf{H}$ are fornally gauge invariant. When we go to compute the $P B$ of $\mathbf{R}$ and $\boldsymbol{H}$ with $\boldsymbol{F}$, we isolate the part of $\mathbf{R}$ and $\boldsymbol{H}$ corresponding to chiral variations $\mathrm{d} \theta$. However, if those chiral variations are along a gauge direction then the corresponding variation of $\mathbf{R}$ and $\mathbf{H}$ is zero by gauge invariance. This would seem to indicate that the naive calculation based on eq.(3.17) was legal. However, $\mathbf{R}, \mathrm{H}$ and $\psi$ are not completely gauge invariant, rather there is gauge dependence at the base point $x_{0}$. When computing the $\mathbf{P B}$, one has to do integration by parts, which give rise to base point contributions that depend on the projection operator $P$ of eq.(3.24). As a result, the PB's of two $\psi$ 's do not close into $\psi$ 's 25 in eq.(3.21), but there are additional unpleasant contributions from the base point. However we do not compute these extra terms explicitly since the advantage of working solely with the one-'body $\psi$ 's would then be lost.

We can avoid these complications by recalling that ultimately we are only interested in the truly gauge invariant observables of eq.(2.15). In computing the PB of two such observ- 
ables, the base point contributions all cancel, since theue are pure gauge terms. Equiviently. we may notice that the gauge invariant observables of eq.(2.15) do not depend on the base point, suggesting that the unwanted terms must cancel. These clearly are not independent arguments, but merely two sides of the same coin. It follows that although eq.(3.21) is not correct as it stands for non-abelian cosets, it is still perfectly alright to use it as an intermediate stz̄p in computing the PB's of gauge invariant quantities, such as those given in eq. (2.15).

Recali that $\psi$ is a coset valued field,

$$
\psi=\psi^{2} r^{2} \quad T^{2} \in \mathbf{g}-\mathbf{h}
$$

This index forms a representation of the subgroup $H$ of $G$. It is useful to think of it as a conserved, non-abelian, global quantum number (color), generated by $\boldsymbol{B}$. The gauge intariant observables (O's) are then the global color-singlet combinations of the parafermions. For these color-singlet observables, it is alright to use eq.(3.21) in computing PB's. To summarize,

a) The color-full parafermions $(\psi)$ are the huilding blocks of color-singlet physical quantities. It is much simpler to work with $\psi$ 's rather than the color-singlet observables directly.

b) The PB's given in eq.(3.21), although not correct for the $\psi$ 's themselves, give the correct result when used in calculating the PB's of color-singlet observables. The extra terms needed to correct eq.(3.21) are contributions from the base point, and thus all cancel when considering the "multilocal" observables of eq.(2.15).

As a check, we have verified that the Jacobj identity is satisfied for the PB algebra of color-singlets (see appendix A). 
From a somewhat different point of view, we may postulate eq.(3.21) as a non-associative algebra between the components of $\psi$. Clearly the correspondence with Poisson brackets is lost, nor is it possible to quantize such an algebra, representing $\psi$ 's as operators in a Hilbert space. However, as stated earlier, if we project onto the color-singlet "multilocal" obseryables defined in eq.(2.15), the algebra is rendered assocjative. We identify this image as the true Poisson algebra, and as we shall show later, this algebra can be quantized consistently.

In a rather different approach the non-associative nature of one-body operators in a confining theory was first considered in ref.[24], where octonians, the simplest realization of a non-associative ring, were postulated as a physical algebra. It is possible that similar nonassociative algebras could be derived from other confining systems such as two dimensional QCD. The existence and classification of this type of nontrivial structure in non-associative algebras may also be interesting from a purely mathematical point of view.

We conclude this section with a generalization of eq.(3.21) to the case where $\mathbf{B}$ is the tensor sum of two affine lie algebras of different central charges. That is,

$$
\mathbf{B}=\mathbf{g} k_{2} \oplus \mathbf{g}_{k_{2}} ; \quad \mathbf{h}=\mathbf{g} k_{\mathbf{1}}+k_{3} \text { the diagonal subalgebra. }
$$

These cosets give an important class of examples, containing the minimal rodels ${ }^{3}$. Calculation of the PB's requires a slight igeneralization of the preceeding calculation. We present only the final result. The coset valued $\psi$ takes the form

$$
\psi=\sum_{a} \psi^{a}\left(\tau_{1}^{a}-\tau_{2}^{a}\right)
$$

We define

$$
P=\sum_{a} P^{a}\left(\tau_{1}^{a}-\tau_{2}^{a}\right) ; \quad \bar{P}=\sum_{a} P^{a}\left(\tau_{i}^{a}+\tau_{2}^{a}\right) .
$$

and present the result

$$
\left\{\psi_{P}, \psi_{Q}\right\}=\frac{4 k_{1} k_{2}}{\pi\left(k_{1}+k_{2}\right)} \int d x \operatorname{tr}\left(P \partial_{x} Q\right)+\frac{4\left(k_{1}-k_{2}\right)}{k_{1}+k_{2}} \int d x \operatorname{tr}(\psi[Q, \bar{P}])
$$




$$
+\frac{2 \pi}{k_{1}+k_{2}} \int d x d y \varepsilon(x-y) \operatorname{tr}([\psi(x), P(x)][\psi(y), Q(y)]) .
$$

Just as in eq. (3.21) this algebra is non-associative due to the neglect of base point contributions. 


\section{IV. "Free Current" Realization of Parafermion Algebra}

We would like to realize the classical algebras given in eq.(3.21) and eq.(3.36) in terms of free fields. We have found that the simplest roite to achieving this is given by a two step process. In the first step we realize the algebres given in eq.(3.21) and eq.(3.36) in terms of a free current algebra or affine algebra. The second step being the wns known realization of aftine Lie algebras by free fields ${ }^{25,26,27}$. That is, we give an explicit construction of the cosel models in terms of currents of the ungauged WZW model. The ccnstruction is very simple, and its quantum extension. discussed in the next section, presents a starting point for constructing the primary fields of the model.

We will start with eq.(3.21) and try to find an explicit constuction for $\psi$ in terms of fields that satisfy simple, local commutation relations (or Poisson brackets). The only consistent local algebras that we are aware of are either the affine algebras or the free field algebras, which can be reguarded as a special case of the former. We cannot hope to reproduce eq.(3.21) exactly, since it is non-associative; however, we can realize eq.(3.21) up to base-point dependent surface terms. We mimic eq.(2.8) by writing

$$
\psi(x)=W^{-1}\left(x, x_{0}\right) E(x) W\left(x, x_{0}\right)
$$

Where $W$ replaces $U$ and $E$ replaces $J . W$ is a bilocal $H$-valued field defined as a path ordered product, as in eq. (2.9)

$$
W\left(x, x_{0}\right)=P_{C} \exp \left(\mathrm{i} \int_{x_{0}}^{x^{\prime}} \mathrm{dx} B\left(x^{\prime}\right)\right)
$$

To proceed we will need the identities

$$
\partial_{x} W(x, y)=i B(x) W(x, y) ; \quad \partial_{y} W(x, y)=-i W(w, y) B(y)
$$

We require $B$ and $E$ to have local PE's. The non-local term of eq.(3.21) is entirely due to the non-locality of the Wilson line $W$. 
It is passibl: to avoid path-dependent terms in the Poisson brackets of the parafermions expressed in eq (4.1) if we require $E$, and $E$ to satisfy an affine algebra with correctly chosen central charge. We introducs the free surrent

$$
\mathcal{F}(x)=r^{l} \mathcal{F}^{l}(x) ; \quad T^{l} \in \boldsymbol{E}
$$

with the following affine algebra

$$
\left\{\mathcal{F}^{k}(x), \mathcal{F}^{\ell}(y)\right\}=-\frac{\alpha}{2} \delta^{\prime}(x-y) \delta^{k \ell}-\frac{i}{4} \delta(x-y) \operatorname{tr}\left(\left[\tau^{k}, \tau^{\ell}\right] \mathcal{F}(x)\right)
$$

Define:

$$
\mathcal{F}_{(h)} \equiv \mathcal{F}^{a} h^{a}, h^{\bullet} \in h ; \mathcal{F}_{(c)} \equiv \mathcal{F}^{l} \mathcal{T}^{l}, \tau^{\ell} \in \mathbf{s}-h .
$$

With these currents defined, we identify

$$
B=\beta \mathcal{F}_{(h)} ; \quad E=\gamma \mathcal{F}_{(c)},
$$

with constants $\alpha_{, r}$. and $\gamma$ to be determined. We need to compute the PB's of $W^{r}$ and $E$. as well as $W$ with itself. in keeping with our policy of suppressing indices, it is useful to introduce the following

$$
\mathcal{F}_{P} \equiv \int d x \operatorname{tr}\left(\mathcal{F}_{(e)}(x) P(x)\right) ; \quad W_{Z} \equiv \int d x \operatorname{tr}\left(W\left(x, x_{0}\right) Z(x)\right)
$$

with $P(x) \in g-h ; Z(x) \in H$. Letting $K$ be an arbitrary dynamical variatle, the necessary for:nulas take the form

$$
\begin{gathered}
\left\{W_{2}, K(y)\right\}=i \beta \int d x \int_{x_{0}}^{x} d x^{\prime}\left\{\mathcal{F}^{a}\left(x^{\prime}\right), K(y)\right\} \operatorname{tr}\left(W\left(x, x^{\prime}\right) h^{a} W\left(x^{\prime}, x_{0}\right) Z(x)\right) \\
\left\{W_{Z_{1}}, W_{Z_{2}}\right\}=-\beta^{2} \int d x \int d y \int_{x_{0}}^{x} d x^{\prime} \int_{x_{0}}^{p} d y^{\prime}\left\{\mathcal{F}^{a}\left(x^{\prime}\right), \mathcal{F}^{b}\left(y^{\prime}\right)\right\} \\
\cdot \operatorname{tr}\left(W\left(x, x^{\prime}\right) h^{a} W\left(x^{\prime}, x_{0}\right) Z_{1}(x)\right) \operatorname{tr}\left(W\left(y, y^{\prime}\right) h^{b} W\left(y^{\prime}, y_{0}\right) Z_{2}(y)\right) .
\end{gathered}
$$

To avoid ill defined terms in eq.(4.10), we have temporarily introduced two distinct base points $x_{0}$ and $y_{0}$. To evaluate eq.(4.10), we substitute the $P B$ given in eq. (4.5), and integrate 
the derivative of the delta function by parts. After some algebra, we arrive at

$$
\begin{gathered}
\left\{W_{Z_{2}}, W_{Z_{2}}\right\}=\frac{\alpha \beta^{2}}{4} \int d x d y\left(\varepsilon\left(x_{0}-y\right)+\varepsilon\left(x-y_{0}\right)-\varepsilon(x-y)-\varepsilon\left(x_{0}-y_{0}\right)\right) \\
\cdot \operatorname{tr}\left(W\left(x, y_{0}\right) h^{a} Z_{1}(x)\right) \operatorname{tr}\left(W\left(y, x_{0}\right) h^{a} Z_{2}(y)\right) \\
+i \frac{\beta^{2}}{2}(\alpha \beta-1) \int d x d y \int d x^{\prime} \operatorname{tr}\left(W\left(x, x^{\prime}\right) h^{a} W\left(x^{\prime}, x_{0}\right) Z_{1}(x)\right) \\
\cdot \operatorname{tr}\left(W\left(y, x^{\prime}\right)\left[h^{a}, \mathcal{F}_{(h)}\left(x^{\prime}\right)\right] W\left(x^{\prime}, y_{0}\right) Z_{2}(y)\right) .
\end{gathered}
$$

The last term, which involves integration over $x^{\prime}$, cannont be reexpressed in terms of $\mathrm{If}^{\prime}$ alone. If this term does not cancel, the PB of two $\psi$ 's will not close. We are forced to require

$$
\alpha \beta^{3}=1 \text {. }
$$

At this point, we drop the terms dependent on the base point. As in the previous section, these terms would have cancelled after projecting onto color-singlet observables. With these simplifications, we have

$$
\left\{W_{Z_{1}}, W_{Z_{2}}\right\} \cong-\frac{1}{4 a} \int d x d y \varepsilon(x-y) \operatorname{tr}\left(W\left(x, x_{0}\right) h^{a} Z_{1}(x)\right) \operatorname{tr}\left(W\left(y, I_{0}\right) h^{\alpha} Z_{2}(y)\right)
$$

Finally; we need to compute the $\mathrm{PB}$ of $E$ with $W$. Employing eq.(4.8), we have

$$
\left\{W_{Z}, \mathcal{F}_{P}\right\}=-\frac{1}{2 a} \int d x d y \operatorname{tr}\left(W(x, y) h^{a} W\left(y, x_{0}\right) Z(x)\right) \operatorname{tr}\left(h^{a}\left[\mathcal{F}_{(\mathrm{c})}(y), P(y)\right]\right) .
$$

Putting together the above relations we obtain the result

$$
\begin{aligned}
& \left\{\psi_{P}, \psi_{Q}\right\}=-\gamma^{2} a \int d x \operatorname{tr}\left(P \partial_{x} Q\right)-i \gamma \int d x \operatorname{tr}([P, Q] \psi(x)) \\
& +\frac{1}{4 a} \int d x d y \varepsilon(x-y) \operatorname{tr}\left(\left[h^{a}, P(;)\right] \psi(x)\right) \operatorname{tr}\left(\left[h^{a}, Q(y)\right] \psi(y)\right) .
\end{aligned}
$$

Taking $\alpha=k / 2 \pi ; \gamma=-2$, we have complete agreement with eq.(3.21). We note that the central charge of the affine Lie algebra eq.(4.5) is the usual quantized value $k / 2 \pi, k \in \mathrm{Z}$. 
as required by unitarity. This construction cain essily be extended to the model defined in eq.(3.34). We define two commuting affina algebras as follow's

$$
\begin{gathered}
\left\{\mathcal{F}_{(1)}^{k}(x), \mathcal{F}_{(1)}^{\ell}(y)\right\}=-\frac{k_{1}}{8 \pi} \delta^{f}(x-y) \delta^{k \ell}-\frac{i}{4} \delta(x-y) \operatorname{tr}\left(\left[\tau_{(1)}^{k}, \tau_{(1)}^{\ell}\right] \mathcal{F}_{(1)}(x)\right) \\
\left\{\mathcal{F}_{(2)}^{k}(x), \mathcal{F}_{(2)}^{l}(y)\right\}=-\frac{k_{2}}{8 \pi} \delta^{\prime}(x-y) \delta^{k \ell}-\frac{i}{4} \delta(x-y) \operatorname{tr}\left(\left[\tau_{(2)}^{k}, \tau_{(2)}^{\ell}\right] \mathcal{F}_{(2)}(x)\right) \\
\left\{\mathcal{F}_{(1)}^{k}(x), \mathcal{F}_{(2)}^{l}(y)\right\}=0 .
\end{gathered}
$$

Defining the diagonal subalgebra $\mathcal{F}=\mathcal{F}_{(1)}+\mathcal{F}_{\{(\})}$, the construction of eq.(4.1) realizes the algebra of eq.(3.37) upon setting

$$
B=\frac{k_{1}+k_{2}}{4 \pi} ; \quad E=\frac{4}{k_{1}+k_{2}}\left(k_{2} \mathcal{F}_{(1)}-k_{1} \mathcal{F}_{(2)}\right) .
$$

In closing this section, we will check two simple consequences of our construction. Consider the simplest gauge intariant function given by

$$
O(x, y)=\operatorname{tr}(\psi(x) \psi(y))={ }_{4} \operatorname{tr}\left(\mathcal{F}_{(c)}(x) W(x, y) \mathcal{F}_{(f)}(y) W(y, x)\right) .
$$

A. explained in ref.[18], we expect to recover the classical stress tensor as $x-y$. This is the classical analogue of the leading term in the operator product expansion. From eq.(4.1), we have

$$
O(x, x) \approx 8 \sum_{l \in \text { eoent }} \mathcal{F}^{l}(x) \mathcal{F}^{l}(x)
$$

Up to an overal normalization constant, this is the classical analogue of the Sugawara constiuction for the coset model.

One other non-trivial check is to verify that all gauge invariant functions lie in the coset. In operator language, they should all commute with currents that belong to the subgroup $H$. The classical analogue is the following PB relation

$$
\left\{O, \mathcal{F}_{[h\}}(z)\right\}=0
$$


for all observables $O$ defined in eq.(2.15). Using eq.(4.13) and eq.(4.14), we have

$$
\left\{W\left(x, x_{0}\right), \mathcal{F}_{(h)}^{a}(z)\right\}=-\frac{i k}{4 \pi}\left(-\delta(x-z) h^{a} W\left(x, x_{0}\right)-\delta\left(x_{0}-z\right) W\left(x, x_{0}\right) h^{a}\right) .
$$

Using this result in eq.(4.17) for $O(x, y)$, it is easy to show that the PB's of W's with $\mathcal{F}_{[h]}$ cancel against the PB's of $\mathcal{F}_{(c)}$ and $\mathcal{F}_{(h)}$. The result generalizes in a straightforward fashion to the complete set of observables given in eq.(2.15). 


\section{Quantiration of the Poisson Algebra}

We would like to generalize the realization of the classical parafermion algebra to a quantum construction. We replace the classical variables $\mathcal{F}^{\prime}(x)$ by operators and the Poisson algebra cq.(4.5) by the corresponding commutator algebra. The resulting expressions are divergent, and regularization and renormalization schemes are necessary. The relation $\alpha=$ $k / 2 \pi$, with $k \in Z$ clearly survives quantization. On the other hand, $\beta$ and $\gamma$ of eq.(4.7) may need renormalization. We will show that the classical relation

$$
\beta=\frac{1}{\alpha}=\frac{2 \pi}{k},
$$

:emains valid to at least first order quantum corrections. This relation may, however, undergo finite renormalization at higher orders. Finally $\mathbf{7}$, which is classically an arbitrary normalization constant, needs infinite renormalization after quantization. We will argue that $\gamma$ is multiplicatively renormalized, and therefore $\psi$ is rendered finite by a multiplicative renormalization constant. As it should be clear from the above discussion, our treatment of the operator construction is still preiiminary and more work needs to be done to put it on a firm foundation.

When we replace the classical variables $\mathcal{F}^{\prime}(x)$ by operators satisfying the affine algebra, we face the question of the ordering of factors in eq.(4.1) and in the definition of $W$ (eq.(4.2)). Even classically, $\mathcal{F}$ is a matrix in color space, where cperator oredering is prescribed by path ordering the exponential. When the classical $\mathcal{F}^{\prime}(x)$ become operators, it is natural to impose the same path ordering for these operators, treating the two spaces as a tensor product. In this way, we also preserve the important semigroup properties (see eq.(2.10)). Clearly $W$ and $\psi$ are riddled with infinities which come from the coalesting of the arguments of two or more $\mathcal{F}$ operators. There are varjous ways of regulating these expressions. For example, one could discretize the space variable " $x "$, and write $W$ as a product. Another method, which we have found more convenient for our calculations, involves dampening the contributing 
modes in momentum space. We express the operator algebra in mode number

$$
\begin{gathered}
{\left[\mathcal{F}_{p}^{k}, \mathcal{F}_{q}^{\ell}\right]=\frac{k}{2} p \delta_{p,-q}+\frac{1}{4} \operatorname{tr}\left(\left[\tau^{k}, \tau^{\ell}\right] \mathcal{F}_{p+q}\right)} \\
\text { with } \mathcal{F}(x)=\frac{1}{2 \pi} \sum_{p} \mathrm{e}^{\mathrm{i} p \cdot x} \mathcal{F}_{p} .
\end{gathered}
$$

where $p$ ranges over the integers, or half integers. To regularize, we replace eq.(5.3)

$$
\text { with } \mathcal{F}(\iota)=\frac{1}{2 \pi} \sum_{p} e^{i p \cdot r-\varepsilon|p|} \mathcal{F}_{p}
$$

The regulator " $\varepsilon^{n}$ is a small positive quantity, and the operators $\mathcal{F}_{p}$ still satisfy the algebra eq.(5.2). However, the affine algebra in position space eq.(4.3) is repalced by a non-local algebra. The realizations given in eq.(4.1) and eq.(4.2) remain unchanged, with the provision that the regulated $\mathcal{F}$ 's be used, and that operators should be path ordered in the same manner as the classical Lie algebra generators.

We first investigate the structure of the divergent terms in $W$. We start with the defining relation eq.(4.2), where $\mathcal{F}_{(h)}$ is given by the regulated expression eq.(5.4). We expect the leading divergence to be present in the vacuum expectation value of $W$. We define the vacuum

$$
\mathcal{F}_{p}^{\ell} \mid 0>=0 \text { for } p>0 \text {. }
$$

Set $\mathcal{F}(\boldsymbol{x})=\mathcal{F}_{(+)}+\mathcal{F}_{(-),}$where ${ }^{n}{ }^{n}\left(n_{-n}\right)$ are positive (negative) frequency parts. (The possible zero mode is not relevant to our discussion). By splitting $\mathcal{F}$ in this way, we can express the vacuum expectation value of $W$ in terms of a differential-integral equation

$$
\partial_{x}\langle 0|W(x, y)| 0\rangle=-\beta^{2} \int_{y}^{x} d x^{\prime}\left\langle 0\left|h^{a} W\left(x, x^{\prime}\right)\left[\mathcal{F}_{(+)}^{a}(x), \mathcal{F}\left(x^{\prime}\right)\right] h^{b} W\left(x^{\prime}, y\right)\right| 0\right\rangle .
$$

where $h^{\alpha} \in \mathbf{h}$. Consider the commutator in the above equation in the limit $\varepsilon-0$. i.e. without the cutoff

$$
\left[\mathcal{F}_{(+)}^{a}(x), \mathcal{F}^{b}\left(x^{\prime}\right)\right]=-\frac{i k}{4 \pi} \partial_{x} \delta^{(+)}\left(x-x^{\prime}\right) \delta^{a b}+\frac{1}{4} \delta^{(+)}\left(x-x^{\prime}\right) \operatorname{tr}\left(\left[h^{a}, h^{b}\right] \mathcal{F}\left(x^{\prime}\right)\right) .
$$


where $\delta(+)$ indicates the positive frequency part of the delta function. We now repeat the classical arguments leading to eq.(4.11) : use integration by parts on the first term of eq. (5.7) with respect to $x^{\prime}$, and when the derivative operator is applied to $W$ use eq.(4.3). Just as in the classical case, the terms obtained in this way cancel against the contribution from the second term in eq.(5.7) if $\alpha \beta=1$. This leaves us with the end point contributions from the limits of integration at $x$ and $y$. This argument breaks down when the regulator $\varepsilon$ is reintroduced; there is no longer a complete cancellation between the first and the second terms of eq.(5.7). However, on intuitive grounds, one may expect the terms singular in $\varepsilon$ as $\varepsilon-0$ will cancel in this way. We show in appendix $B$ that this expectation is justified to at least the lowest order in $1 / \mathrm{k}$. What happens at higher orders is an open question. However, assuming that the singular terms continue to cancel, we have the following result

$$
\partial_{x}<0|W(x, y)| 0>\cong-\frac{i \pi}{k} C \delta_{e}^{(+)}(0)<0|W(x, y)| 0>,
$$

where $\mathrm{C}$ is the Casimir of the subgroup $H$

$$
\sum_{0} h^{0} h^{0}=\mathrm{C} I_{;} \quad I=\text { identity matris, }
$$

and $\delta_{c}^{t+1}$ is the regulated delta function

$$
\delta_{\varepsilon}^{(+1}(x)=\frac{1}{2 \pi} \sum_{p=0}^{\infty} e^{i p \cdot x-p_{j}} \delta_{\varepsilon}^{(+)}(0)=\frac{1}{2 \pi} \frac{1}{1-e^{-\pi}} \cong \frac{1}{2 \pi \varepsilon} .
$$

Ln deriving eq.(5.8), we have only retained terms singular as $\varepsilon \rightarrow 0$, which come from integrating $\partial_{x} \delta^{(+)}\left(x-x^{\prime}\right)$ with respect to $x^{\prime}$ and pjcking up the end point contributions at $x=x^{\prime}$. Integrating eq. $(5.8)$, we find the following singularity structure

$$
<0|W(x, y)| 0>\cong \exp \left(-\frac{i C}{2 k \varepsilon}(x-y)\right) \text {. finite factor . }
$$

This formula supports our contention that the only infinite rebormalization needed is a multiplicative one. Physically, the result is quite plausible : the Wilson line carties an 
infinite amount of energy per unit length due to its infinitesimal thickness

$$
\text { energy per unit length }=\frac{C}{2 k \varepsilon} .
$$

This result is valid to lowest order in $1 / \mathbf{k}$; it may well be modified at higher order.

Finally, we would like to mention that the quantum analogue of eq. (4.20) can also be derived, subject to the assumptions leading to eq.(5.8). 


\section{vI. Conclusion:}

In this paper, we have extended the results of our earlier work on abelian coset models to non-abelian cosets. As before, starting with the gauged WZW lagrangian, we hase shown how to define the parafermion fields and derived their Poisson bracket algebra. As explained in the text, we were naturally led to introduce a non-associative algebra for the parafermions which reduces to an associative algebra in the physical (gauge invariant) sector. We have also given a simple construction for this sector in terms of free currents that satisfy a classical affine algebra. Replacing the classical current algebra by its quantum version gives a fully quantur mechanical construction, which is the natural generalization of the free field realization of the abelian coset models. We have also taken the initial steps in carrying out the program of renormalizing the quantum construction. Several problems remain for the future, the most important one being the question of renormalization. Also, generalization to non-compact cosets should be stringhtforward, perhaps making it possible to investigate the unitarity of these models ${ }^{30}$. Finally, it remains to be seen whether more general conformal models ${ }^{31}$ can be discussed within the framework introduced here. 


\section{Acknowledgement:}

One of the authors (K.B) would like to thank E.Rabinovici for stimulating discussions and hospitality at the Hebrew University in Jerusalem, where this project started during a visit supported by American-Israeli Binatioal Foundation. This work is supported in part by the director, Office of Energy Research, Office of High Energy and Nuclear Physics, Division of High Energy Physics of the U.S. Department of Energy under Cotract DE-AC0376SF00098 and in part by the National Science Foundation under grant PHY85-1585i. Another author (M.C) was supported by a National Science Foundation Fellowship ano a Department of Education Grant. 


\section{Appendix A}

We show that the algebra given in eq.(3.21) satisfies the Jacnbi identity eq.(3.22) after projecting onto color-singlet observables. When we substitute eq.(3.21) into eq.(3.22), we will get an expansion in $k^{i}$, with $i=-2,-1,0,1,2$. It is not difficult to show that all of these terms sancel even for single. color-full parafermions, with the exception of $i=0$ and $i=-2$. The term independent of $k(j=0)$ has two contributions. One term where the $k$-independent term of the PB is used twice, and one where the non-lecal term proportional to $\varepsilon(x-y)$ is in the inner PB, and then the "central charge" term is used in the outer PB. These terms combine to give a restatement of the Jacobi identity for the underlying semisimple Lie algebra, and in this manner give a nontrivial restriction on the coefficients of the $P B$ of eq.(3.21). If we label the coefficients of the three terms of eq.(3.21) as a,b,c respectively: this restriction takes the form (where the normalization of the trace takes the customary form $\left.\operatorname{tr}\left(\tau^{a} \tau^{b}\right)=2 \delta^{a b}\right)$

$$
4 a c=-b^{2}
$$

which is satisfied in our case. Let this expression fix $\mathrm{c}$ in terms of $\mathbf{a}$ and $\mathbf{b}$. We can arbitrarily scale the parafermions $\psi$ to fix b. This leaves a single overall normalization constant for the right hand side of the Poisson algebra. We now concentrate on the leading term $(\mathrm{i}=$ $.2)$, and work with the truncated algebra

$$
\left\{\psi_{P}, \psi_{Q}\right\}=\int d x d y \leq(x-y) \sum_{a} \operatorname{tr}\left(P(x)\left[h_{a}, \psi(x)\right]\right) \operatorname{tr}\left(Q(x)\left[h_{a}, \psi(x)\right]\right),
$$

the sum being carried over the subalgebra $h$. With $O(x)=O\left(x_{1}, x_{2}, \ldots, x_{n}\right)$ defined in eq.(2.15), we wish to show that

$$
\sum_{\text {ryclic }}\left\{\left\{O_{1}(x), O_{2}(y)\right\}, O_{3}(z)\right\}=0
$$

Define:

$$
X_{i}^{a} \equiv \operatorname{Tr}\left(\psi\left(x_{1}\right) \cdots\left[h^{a}, \psi\left(x_{i}\right)\right] \cdots \psi\left(x_{n}\right)\right)
$$


and similarly for $Y$ and $Z$ (involving $\psi\left(y_{i}\right)$ 's and $\psi\left(z_{i}\right)$ 's repectively). We have

$$
\left\{O_{1}(x), O_{2}(y)\right\}=\varepsilon\left(x_{i}-y_{j}\right) X_{i}^{a} Y_{j}^{a}
$$

where " $a$ " is summed over the subalgebra $h$, and the dummy indices " ${ }^{n}$ " and " $j^{n}$ are summed over the multivariable index range of ${ }^{n} x^{n}$ and ${ }^{n} y^{n}$ respectively. We can write

$$
\begin{gathered}
\left\{\left\{O_{1}(x), O_{1}(y)\right\}, O_{1}(z)\right\}=\varepsilon\left(x_{i}-y_{j}\right)\left(X_{i k}^{a b} Y_{j}^{a} Z_{k}^{b} \varepsilon\left(x_{l}-z_{k}\right)+X_{i}^{a} Y_{j l}^{a b} Z_{k}^{b} \varepsilon\left(y_{l}-z_{k}\right)\right) \\
\text { where } X_{i j}^{a b} \equiv\left\{\begin{array}{c}
\operatorname{tr}\left(\dot{U}\left(x_{1}\right) \cdots\left[h^{a}, \psi\left(x_{i}\right)\right] \cdots\left[h^{b}, \psi\left(x_{j}\right)\right] \cdots \psi\left(x_{n}\right)\right) i \neq j \\
\operatorname{tr}\left(\psi\left(x_{1}\right) \cdots\left[h^{a},\left[h^{b}, \psi\left(x_{i}\right)\right]\right] \cdots \psi\left(x_{n}\right)\right) i=j
\end{array}\right.
\end{gathered}
$$

$$
X_{i j}^{a b} \text { sastisfies the identity: } X_{i j}^{a b}-X_{j i}^{b a}=\left\{_{f_{\infty}}^{0} X_{i}^{c} \underset{i=j}{i \neq j}\right.
$$

where $f_{a b c}$ are the structure constants of the Lie algebra 8 . Since we are summing over permutations, we are free to permute the second term of eq.(A.5) twice in $(x, y, 2)$. After rearranging dummy indices, and employing eq.(A.6), this expression reduces to

$$
f_{a b e} X_{i}^{a} Y_{j}^{b} Z_{k}^{c} \varepsilon\left(x_{i}-y_{j}\right) \varepsilon\left(x_{i}-z_{k}\right)
$$

we sum permutations, and rewrite dummy indices to arrive at

$$
\left\{\left\{O_{1}, O_{2}\right\}, O_{3}\right\}=f_{a b c} X_{i}^{a} Y_{j}^{b} Z_{k}^{c}\left\{\varepsilon\left(x_{i}-y_{j}\right) \varepsilon\left(x_{i}-z_{k}\right)+\varepsilon\left(y_{j}-z_{k}\right) \varepsilon\left(y_{j}-x_{i}\right)+\varepsilon\left(z_{k}-x_{k}\right) \varepsilon\left(z_{k}-y_{j}\right]\right.
$$

Now employing the identity

$$
\varepsilon(x-y) \varepsilon(x-z)+\varepsilon(y-z) \varepsilon(y-x)+\varepsilon(z-x) \varepsilon(z-y)=1 \quad \forall x, y, z \in \mathbf{R},
$$

we bave

$$
\sum_{\text {cydlic }}\left\{\left\{O_{1}(x), O_{2}(y)\right\}, O_{3}(z)\right\}=\sum_{i, j, k} f_{a b c} X_{i}^{a} Y_{j}^{b} Z_{k}^{c}=\sum_{j, k} f_{a b c} Y_{j}^{b} Z_{k}^{c} \sum_{i} X_{i}^{a}
$$


By color neutrality, the sum over ${ }^{\prime \prime}$ on the right hand side of eq. $\{$ A.10) vanishes, giving the desired result. We may also notice that if we had not used color-singlet observables, the Jacobi identity would not have been satisfied in general, but if just one of the observables is a color singlet, the Jacobi identity is satisfied. 


\section{Appendix B}

In this appendix, we verify the cancellation discussed after eq.(5.7), to lowest order in 1/k. First, expand $W$ in a power series,

$$
W=\sum_{n}\left(\frac{1}{k}\right)^{n} W_{n},
$$

where $W_{n}$ takes the form

$$
W_{n}=\frac{1}{n !} \prod_{i=1}^{n} \int_{v}^{x} d z_{i} \prod_{j=1}^{n} \sum_{p_{1}} \exp \left(\mathrm{i} \sum p_{i} z_{i}-\varepsilon \sum\left|p_{i}\right|\right) \mathcal{F}_{p_{1}} \cdots \mathcal{F}_{p_{2}} .
$$

The cancellation referred to above takes place between $W_{n}$ and $W_{n-1}$; the c-number con* tractions between $\mathcal{F}^{\prime} \mathrm{s}$ in $W_{n}$ (first term of eq. (5.2)) cancel against the operator contractions (second term of eq.(5.2)) of $W_{n-1}$. Notice that these terms are of the same order in $1 / k$. To illustrate the cancellation, consider the simpie example of $W_{4}$ and $W_{3}$. As part of this calculation, consider the c-number contraction between $\mathcal{F}_{p l}$ and $\mathcal{F}_{p 3}$

$$
\begin{gathered}
W_{4} \sim \int_{y}^{z} d z_{4} \int_{\nu}^{z_{4}} d z_{3} \int_{y}^{x_{3}} d z_{2} \int_{\nu}^{z_{3}} d z_{1} \sum_{p_{1} p_{203 p_{4}}} \operatorname{erp}\left(i \sum p_{i} z_{i}-\varepsilon \sum\left|p_{i}\right|\right) \\
\cdot \theta\left(p_{1}\right) p_{1} \delta_{p_{1}+p_{1}} h^{\alpha} \mathcal{F}_{p_{2}} h^{a} \mathcal{F}_{p_{4}},
\end{gathered}
$$

where we have suppressed some irrelevant multiplicative factors. Setting $p_{1} \sim i \partial / \partial z_{3}$ and integrating by parts, we obtain

$$
\begin{gathered}
W_{1} \sim \int_{v}^{x} d z_{3} \int_{v}^{x_{3}} d z_{2} \int_{v}^{x_{2}} d z_{1} \sum_{p_{1} p_{p_{3}}} \theta\left(p_{1}\right) \exp \left(i \sum p_{i} z_{i}-\varepsilon\left(2 p_{1}+\left|p_{1}+p_{2}\right|+\left|p_{3}\right|\right)\right) \\
\cdot h^{a} \mathcal{F}_{p_{1}+p_{2}} h^{a} \mathcal{F}_{p_{1}} .
\end{gathered}
$$

Now compare this result with the q-number contraction between $\mathcal{F}_{\mathrm{m}}$ and $\mathcal{F}_{\mathrm{m}}$ in $W_{3}$

$$
W_{3} \sim-\int_{\nu}^{x} d z_{3} \int_{\nu}^{x_{3}} d z_{2} \int_{y}^{x_{2}} d z_{1} \sum_{p_{1} p_{23} p_{3}} \theta\left(p_{1}\right) \exp \left(i \sum p_{i} z_{i}-\varepsilon \sum\left|p_{i}\right|\right)
$$




$$
\cdot h^{a} \mathcal{F}_{p_{1}+p_{2}} h^{a} \mathcal{F}_{p 3}
$$

Notice that the two terms given by eq.(B.4) and eq.(B.5) would have cancelled except for differert dependence on the cutoff $\varepsilon$. To see what happens as $\varepsilon \rightarrow 0$, take the vacuum expectation values of $W_{3}$ and $W_{4}$

$$
\begin{aligned}
<0]\left(W_{3}+W_{4}\right) \mid 0>\sim & \sum_{p, q>0}\left(\frac{p+q}{q p^{2}}+\frac{i(x-y)}{p}+\frac{q}{p^{2}(q-p)} e^{-i p(x-y)}-\frac{1}{(q-q) q} e^{-i q(x-y)}\right) \\
p \neq 0 & \cdot(\exp (-s(p+q+|p+q|))-\exp (-2 q(p+q))) .
\end{aligned}
$$

A straightforward examination of this sum shows that as $\varepsilon \rightarrow 0$, it is non-singular if the difference $x-y$ is held fixed at some finite value. This demonstrates that the singular terms in $\varepsilon$ present in the cuntraction of $W_{3}$ and $W_{4}$ cancel. The only singular term that survives is an end point contribution given by eq.(5.11). 


\section{Reference:}

1. P.Goddard,A.Kent and D.Clive, Phys. Lett. B152, 88 (1985).

2. K.Bardakci and M.B.Halpern, Phys. Rev. D3, 2493 (1971).

3. M.B.Halpern, Phys. Rev. D4, 2398 (1971).

4. A.Belavin, A.M.Polyakov and A.B.Zamolodchikov, Nucl. Phys. B241, 33 (1984).

5. D.Friedan, Z.Qiu and S.Shenker. Phys. Rev. Lett. 52, 1575 (1984).

6. For a review of conformal field theories, see: G.Moore and N.Seiberg, Lectures on RCFT, RU-89-32(Sep. 1989)

7. I.Antoniadis and C.Bachas, Nucl. Phys. B278, 343 (1986).

8. E.Witten, Comm. Math. Phys. 92, 455 (1984).

9. A.M.Polyakov and P.B.Wiegmann, Phys. Lett. B131, 121 (1983); Phys. Lett. B141, $223(1984)$.

10. K.Baraakci, E.Rabinovici and B.Saring, Nucl. Phys. B289, 151 (1988).

11. W.Nahm, Duke Math. J. 54, 579 (1987).

12. A.Kent, Phys. Lett. B173, 413 (1989).

13. K.Gawedzki and A.Kupiainen, Nucl. Phys. B320, 625 (1989).

14. D.Karabali and H.J.Schnitzer, Nucl. Phys. B328, 649 (1990).

15. D.Bernard G.Felder, Comm. Math. Phys. 127, 145 (1990).

16. P.Bouwknegt, J.Mcarthy and K.Pilch, Phys. Lett. B234, 297 (1990).

17. P.Bowcock, Nucl. Phys. B316, 80 (1989). 
18. K.Bardakci, M.Crescimanno and E.Rabinovici, Nucl. Phys. B344, 344 (1990)

19. A.B.Zamolodchikov and V.A.Fateev, Sov. Phys. J.E.T.P. 62, 215 (1985).

20. P.Goddard and A.Schwimmer, Phys. Lett. B208, 62 (1988).

21. A.Gerarimov, A.Marshakov, A.Morozov, M.OLshanetsky and S.Shatarbvili, Int. J. Mod. Phys. A5, 2495 (1990); A.Gerasimov, A.Marshakov and A.Morozov, Nucl. Phys. B328, 664 (1989).

22. K.Gawedski. Nucl. Phys. B328, 733 (1989).

23. G.Dunne, I.Halliday and P.Suranyi, Nucl. Phys. B325, 526 (1989).

24. M.Gunaydin and F.Gursey, Phys. Rev. 8, 3387 (1973).

25. M.Wakimoto, Comm. Math. Phys. 104, 605 (1986).

26. D.Nemeschansky, Phys. Lett. B224, 121 (1989).

27. A.Bilal, Phys. Lett. B226, 272 (1989).

28. V.G.Kac, Func. Anal. App. 1, 328 (1967); R.V.Moody, Bull. Am. Math. Soc. 73. 217 (1967).

29. V.S.Dotsenko and V.A.Fateev, Nucl. Phys. B240, 312 (1984).

30. L.J.Dixon, M.E.Peskin and J.Lykken, Nucl. Pbys. B325, 329 (1989);

I. Bars, Nucl. Phys. B334, 125 (1990).

31. M.B.Halpern and E.Kiritsis, Mod. Phys. Lett. A4, 1373 (1989);

M.B.Halpern, E.Kiritsis, N.A.Obers, M.Porrati and J.P.Yamron, Int. J. of Mod. Phys. A5, 2275 (1990). 


\section{Contributions and Interesta}

This work is the logical extension of the previous paper to the case of a non-abelian coset. The initial set up of the problem thus borrows hexyily upon the formalism of the previous work and besides checking many of the equations in the front sections, this authors' main contribution to this work is in section IV where some of the set up of eq.(4.3) through eq.(4.11) is displayed and again in section $V$ where this author contributed to the discussion of how to regulate these path-ordered exponentials as quantum operators and in particular to the ideas and eq.(5.8) to eq.(5.12). Also much effort was made by this author to formulate the proof of the closure of the (classical) algebra on the space of colorless operators that is described in appendix $A$.

There are certain topics of interest not fully covered in this paper which interest this author deeply. The most fundamental is simply in what way can one regulate path-ordered exponentials to consider them as quantum operators. The present work only approaches this idea which seems to be a central one for understanding this lagrangian approach to conformal field theories. One is also forced to understand the meaning of path-ordered exponentials of gauge fields as quantum operators to really fully understand the connection between conformal field theory and Chern-Simons field theory. As of this writing this is an ongoing project of this auinor. 


\section{CHAPTER 4: Classical W-Algebras and Non-Abelian Coset Models}

Coset models often furnish examples of conformal field theories with a group of symmetries larger than the conformal group. This larger symmetry algebra, the so called W-algebra or extended conformal algebra, may be understood classically in a universal way from the Pojsson algebra of the coset models developed in Chapter 3.

This paper concisely shows how to understand the universal features of $\mathrm{W}$-algebras from the Poisson structure of the underlying coset model. It also describes a natural generalization of the $W$-algebras that is an algebra of multilocal operators. 
Classical W-Algebras and Non-Abelian Parafermions

\author{
K. Bardakei \\ M. Crescimanno \\ and \\ S.A. Hotes \\ Department of Physics \\ University of California \\ Berkeley, Ca. 94720 \\ and \\ Laurence Berkeley Laboratory \\ to appear in Physics Review Letters
}


Recently there has been much interest in $W$ algebras-8. These algebras are generated in the operator product expansion of the primary fields of rational conformal models, and can be considered to be natural generalizations of the Virasoro algebra. The classical limits of these algebras are of particular interest ${ }^{2}$, and in this paper, we present a simple method of generating a large class of classical $W$ algebras. This clas includes the already well-known $W_{3}$ and $W_{1}$ algebras as well as some new algebras which, to our knowledge, have not yet appeared in the literature. We show that the generators of these algebras can be built out of parafermion fields introduced in ref.[9,10]. The parafermion fields themselves satisfy a very simple Poisson bracket algebra, which turns out to be the basic irreducible structure out of which one can build the more complicated algebras. As a result, a great simplification is achieved, if only at the classical level. - In this sense, the parafermions are analogous to quarks and the primary fields are the composite fields formed from the "quarks". The ajgebra of "quarks" is, not surprisingly, much simpler than the algebra generated by the composite operators. We will then go on to show that the parafermion algebra suggests a natural generalization of $W$ algebras that involves multi-local operators.

Let us focus on those conformal field theories that can be described in terms of gauged WZW models. As is well known, after integrating over the gauge field we will be left with a conformal model defined over a reduced set of fields. These models are refered to as coset models and in what follows we will consider coset models of a very particular type: we take $g$ to be the direct sum of two copies of an affine lie algebra with arbitrary central charges $k_{1}$ and $k_{2}$, and take $h$ to be the diagonal subalgebra

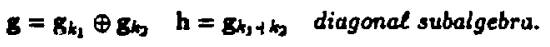

This choice is dictated wolely by the fact that these cosets have been discussed at length in many previous works $3,5,0,4$ and much of what we will do here can be easily generalized to 
other types of coset models. The WZW action corresponding to the coset of eq.(1) is

$$
\begin{gathered}
I=I_{1}+I_{2}+I_{1,2} \\
I_{1}=\frac{k_{1}}{4 \pi} \int d^{2} x \operatorname{tr}\left(\partial_{+} g_{1}^{-1} \partial_{-} g_{1}\right)+\frac{k_{1}}{24 \pi} \int \operatorname{tr}\left(d g_{1} g_{1}^{-1}\right)^{3}, \\
I_{2}=\frac{k_{2}}{4 \pi} \int d^{2} x \operatorname{tr}\left(\partial_{+} g_{2}^{-1} \partial_{-} g_{2}\right)+\frac{k_{2}}{24 \pi} \int \operatorname{tr}\left(d g_{2} g_{2}^{-1}\right)^{3} \\
I_{1,2}=\frac{1}{8 \pi} \int d^{2} x \operatorname{tr}\left\{i A_{+}\left(k_{1}\left(\partial_{-} g_{1}\right) g_{1}^{-1}+k_{2}\left(\partial_{-} g_{2}\right) g_{2}^{-1}\right)-i A_{-}\left(k_{1} g_{1}^{-1} \partial_{+} g_{1}+k_{2} g_{2}^{-1} \partial_{+} g_{2}\right)\right. \\
\left.+k_{1} A_{+} g_{1} A_{-} g_{1}^{-1}+k_{2} A_{+} g_{2} A_{-} g_{2}^{-1}-\left(k_{1}+k_{2}\right) A_{+} A_{-}\right\}
\end{gathered}
$$

Here $g_{1}$ and $g_{2}$ take values in the adjoint representation of some lie algebra, and the gauge field $\boldsymbol{A}_{ \pm}$takes values in $\mathrm{h}$, the vector sum of these two representations. Note however that the eurrents of this model

$$
\begin{gathered}
J_{+}=\frac{i k_{2}}{\pi} g_{1}^{-1} D_{+} g_{1}+\frac{i k_{2}}{\pi} g_{2}^{-1} D_{+} g_{2} \\
J_{-}=-\frac{i k_{1}}{\pi}\left(D_{-} g_{1}\right) g_{1}^{-1}-\frac{i k_{2}}{\pi}\left(D_{-} g_{2}\right) g_{2}^{-1}
\end{gathered}
$$

with

$$
D_{ \pm}=\partial_{ \pm}-i\left[A_{ \pm}\right.
$$

are neither chirally conserved nor gauge invariant. It is possible to construet "almost" conserved and "almost" gauge invariant fields by attaching Wilson lines to the currents: these are the "parafermions" of ref. $[9,10]$. For simplicity we shall consider onily $J_{-}$and drop the lorentz index. Thus with $x=x_{-}$take

$$
\psi=\psi_{-}\left(x, I_{0}\right)=-U^{-1}\left(x, I_{0}\right) J_{-}(x) U\left(x, I_{0}\right),
$$


where

$$
U\left(x, x_{0}\right)=\operatorname{Pexp}\left(i \int_{x_{0}}^{x} \mathrm{~d} x^{\prime} A_{-}\left(x^{\prime}\right)\right),
$$

is the path-ordered Wilson line. One can easily show that

$$
a_{+} \psi_{-}=0
$$

provided that in applying the equations of motion the contributions from the (fixed) base point are neglected. Also, $\psi$ is "almost" gauge invariant in that it transforms only under the global group defined as gauge transformations evaluated at the base point $I_{0}$. In what follows we will think of this base point as a point at infinity. The "physical" fields are constructed by forming invariant (i.e. singlet) combinations of the parafermions under the remaining global color group h described above. They are (classically) base point independant and therefore truly conserved as well as gauge invariant. They are the classical primary fields of the model and we wish to show that their Poisson brackets close to form a classical W algebra. These algebras are generated by the basic Poisson brackets of the $\psi$ 's which may be found by studying the lagrangian eq.(2) (see ref. $\{9,10]$ for a derivation). To avoid confusion we use $\{$,$\} for the Poisson bracket and reserve [,] for Lie algebra commutators.$ With

$$
\psi(x)=\sum_{a} \psi^{a}(x) r^{a}
$$

we have

$$
\begin{aligned}
& \left\{\psi^{a}(x), \psi^{b}(y)\right\}_{P B}=-\frac{2 k_{1} k_{b}}{\pi\left(k_{1}+k_{2}\right)} \delta^{a b} \delta^{\prime}(x-y) \\
& +\frac{\left(k_{1}-b_{2}\right)}{\left(k_{1}+k_{2}\right)} \delta(x-y) \sum_{c} f^{a b c} \psi^{c}(x) \\
& -\frac{\pi}{\left(x_{1}+h_{0}\right)} \varepsilon(x-y) \sum \text { fadefbec }^{\mathrm{d}}(x) \psi^{*}(y)
\end{aligned}
$$

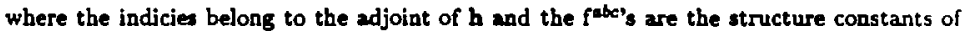
h. The first two terms are familiar from affine (current) algebras and the last term reflects 
the non-locality due to the Wilson line attached to each field. In computing the Poisson bracket of eq.(6) the base point contributions have again been neglected. This has the peculiar effect that the resulting algebre seems not to be associative (the Jacobi identity is not satified). However, as shown explicitely in ref.[10], the color singlet composites built out of the $\psi$ 's do satisfy an associative algebra. This is expected since these functions do not depend on the base point classically.

The simplest neutral combination one can form is obtained by tracing an arbitrary power of $\psi$. Switching to mode number and scaling appropriately to conform to the literature we have

$$
W_{m}^{(s)}=\frac{1}{i a s} \int_{0}^{i \pi} d x e^{i m x} \operatorname{Tr}\left(\psi^{\prime}(x)\right),
$$

where for convenience we set

$$
\alpha=\frac{4 k_{1} k_{2}}{\pi\left(k_{1}+k_{2}\right)} ; \quad \beta=\frac{4\left(k_{1}-k_{2}\right)}{\left(k_{1}+k_{2}\right)} ; \quad \gamma=\frac{2 \pi}{\left(k_{1}+k_{2}\right)}
$$

For concreteness we take $g=s u(N)$ and colapuie the Poisson brackets of the operators in eq. (7) from eq.(6)

$$
\begin{gathered}
\left\{W_{m}^{(s)}, W_{n}^{\left(s^{\prime}\right)}\right\}=\left(m\left(s^{\prime}-1\right)-n(s-1)\right) W_{m+n}^{\left(s+s^{\prime}-2\right)} \\
-\frac{i a(e-1)\left(s^{\prime}-1\right)}{2 \pi N} \sum_{j} j W_{m-j}^{(s-1)} W_{n+j}^{\left(a^{\prime}-1\right)}
\end{gathered}
$$

In the limit $\hat{N} \rightarrow \infty$ only the first term survives and we have the su( $\infty)$, e.g., $W_{\infty}$ algebra. For finite values of $\mathrm{N}$ both terms are present and furthermore all $W^{(0)}$ for $s>n$ car be expressed in terms of $W^{\left(s^{\prime}\right)}$ for $1<s^{\prime} \leq N$. For example in the case of su(3) we have

$$
W_{m+n}^{(4)}=\frac{i a}{4 \pi} \sum_{j} W_{m-j}^{(2)} W_{n+j}^{(2)}
$$

which follows from the trace identity

$$
\operatorname{tr}\left(\psi^{4}\right)=\frac{1}{2}\left(t r\left(\psi^{2}\right)\right)^{2} \quad \text { valid for traceless } 3 \times 3 \text { matrices }
$$


We therefore find the closed algebra

$$
\begin{gathered}
\left\{W_{m}^{(2)}, W_{n}^{(2)}\right\}=(m-n) W_{m+n}^{(2)}, \\
\left\{W_{m}^{(2)}, W_{n}^{(3)}\right\}=(2 m-n) W_{m+n}^{(3)}, \\
\left\{W_{m}^{(3)}, W_{n}^{(3)}\right\}=\frac{i g}{\delta \pi}(m-n) \sum \ell W_{m-\ell}^{(2)} W_{n+\ell}^{(2)}
\end{gathered} .
$$

This agrees with the classical limit of the quantum $W_{3}$ algebra in which one retains only the term with the least singular short-distance behavior. A similar algebra can be deduced for $H_{4}$. We note that these ajgebras bave the simple universal form given in ref.[2]. For finite $N$, we see that the $W_{N}$ aggebra is nonlinear. This is simply due to structure constant identities used to reduce algebra elements $W_{(s)}$ for $s>N$. It is interesting to note that these algebras are insensitive to the second two terms of the algebra given in eq.(6). We now turn to a generalized algebra that will involve the later terms of eq.(6).

We now discuss a generalization of $W$ algebras that is suggested by analyzing $W ' Z W$ models in terms of parafermions. The Poisson algebra of eq.(6) may be used to compute Poisson brackets of gauge-invariant, multilocal functions. As described in ref.[10], the resulting Poisson bracket does satisfy the Jacobi identity. In analogy to eq.(7) consider multilocal functions of the general form

$$
W^{\prime}\left(x_{1} x_{2} \ldots x_{n}\right)=\operatorname{tr}\left(\psi\left(x_{1}\right)\left[\psi\left(x_{2}\right),\left[\psi\left(x_{3}\right), \ldots \psi\left(x_{n}\right)\right] \ldots\right]\right)
$$

This is a natural generalization of eq.(7). As we saw earlier, the fact that the fields in eq. ( 7$)$ are all taken at the same point makes any Poisson bracket of these quantities not depend on the second and third terms of eq.(6). For multilocal inctions the Poisson brackets will involve the second and third terms in a non-trivial way. Thus, although the $k$-dependance of the Poisson bracket may be scaled out in the local case, there will be residual $k$-dependance in the algebra of multilocal expressions.

We now illustrate this non-local algebra with a simple example. Consider the case of 
$\left(S U(2)_{k_{1}} X S U(2)_{k_{2}}\right) / S U(2)_{k_{1}+k_{2}}$. In this case, the aigebra closes on the first two functions

$$
\begin{gathered}
W\left(x_{1} x_{2}\right)=\operatorname{tr}\left(\psi\left(x_{1}\right) \psi\left(x_{2}\right)\right) \\
W\left(x_{1} x_{2} x_{3}\right)=\operatorname{tr}\left(\psi\left(x_{1}\right)\left[\psi\left(x_{2}\right), \psi\left(x_{3}\right)\right]\right)
\end{gathered}
$$

We have the following algebra

$$
\begin{gathered}
\left\{W\left(x_{1} x_{2}\right), W\left(y_{1} y_{2}\right)\right\}=-\alpha \delta^{\prime}\left(x_{1}-y_{1}\right) W\left(x_{2} y_{2}\right)+\text { perm. } \\
-i \beta \delta\left(x_{1}-y_{1}\right) W\left(x_{1} x_{2} y_{2}\right)+p e r m .+2 \gamma \theta\left(x_{1} x_{2} ; y_{1} y_{3}\right) W\left(x_{1} x_{2} y_{1} y_{2}\right) \\
\left\{W\left(x_{1} x_{2}\right), W\left(y_{1} y_{2} y_{3}\right)\right\}=-\alpha \delta^{\prime}\left(x_{1}-y_{1}\right) W\left(x_{2} y_{2} y_{3}\right)+\text { perm. } \\
-i \beta \delta\left(x_{1}-y_{1}\right) W\left(x_{1} x_{2} y_{2} y_{3}\right)+\text { perm. } \\
+2 \gamma \theta\left(x_{1} x_{2} ; y_{1} y_{3}\right) W\left(x_{1} x_{2} y_{1} y_{2} y_{3}\right)+2 \gamma \theta\left(x_{1} x_{2} ; y_{2} y_{3}\right) W\left(x_{1} x_{2} y_{2} y_{3} y_{1}\right) \\
\left\{W\left(x_{1} x_{2} x_{3}\right), W\left(y_{1} y_{2} y_{3}\right)\right\}=-\alpha \delta^{\prime}\left(x_{1}-y_{1}\right) W\left(x_{2} x_{3} y_{2} y_{3}\right)+\text { perm. } \\
+i \beta \delta\left(x_{1}-y_{1}\right) W\left(x_{2} x_{3} y_{1} y_{2} y_{3}\right)+p e r m . \\
+2 \gamma \theta\left(x_{1} x_{3} ; y_{1} y_{3}\right) W\left(x_{3} x_{2} x_{1} y_{1} y_{2} y_{3}\right)+2 \gamma \theta\left(x_{2} x_{3} ; y_{1} y_{3}\right) W\left(x_{2} x_{3} x_{1} y_{1} y_{2} y_{3}\right) \\
+2 \gamma \theta\left(x_{1} x_{3} ; y_{2} y_{3}\right) W\left(x_{3} x_{2} x_{1} y_{2} y_{3} y_{1}\right)+2 \gamma \theta\left(x_{2} x_{3} ; y_{2} y_{3}\right) W\left(x_{2} x_{3} x_{1} y_{2} y_{3} y_{1}\right)
\end{gathered}
$$

We have indicated by "perm." the terms obtained by symmetrizing or antisymmetrizing with respect to the arguments in the appropriate way. Note that $W\left(x_{1} x_{2}\right)$ is symmetric in $x_{1}$ and $x_{2}$, and that $W\left(x_{1} x_{2} x_{3}\right)$ is antisymmetric in $x_{1}, x_{2}$ and $x_{3}$. The $\theta$ function used above is defined as

$$
2 \theta\left(x_{1} x_{2} ; y_{1} y_{2}\right)=\varepsilon\left(x_{1}-y_{1}\right)+\varepsilon\left(x_{2}-y_{2}\right)-\varepsilon\left(x_{1}-y_{2}\right)-\varepsilon\left(x_{2}-y_{1}\right)
$$

and measures the overlap between the intervals $\left[x_{1}, x_{2}\right]$ and $\left[y_{1}, y_{2}\right]$. If one interval contains the other or if they are disjoint, it vanishes. If the intervals overlap, $\theta$ is \pm 1 depending on the sense of the overiap. It is simple to extract a truly local algebra from the multi-local functions by expanding in power series in the differences of arguments, as in the operator product expansion. Finally, we need to show that the functions appearing on the RHS of eq.(14), eq.(15), and eq.(16) can be written in terms of the functions given in eq.(13). This 
follows from elementary identities beíween su(2) structure constants. For example,

$$
\begin{gathered}
W\left(x_{1} x_{2} x_{3} x_{4}\right)=\frac{1}{2} W\left(x_{2} x_{3}\right) W\left(x_{1} x_{4}\right)-\frac{1}{3} W\left(x_{1} x_{3}\right) W\left(x_{2} x_{4}\right) \\
W\left(x_{1} x_{2} x_{3} x_{4} x_{5}\right)=\frac{1}{2} W\left(x_{3} x_{4}\right) W\left(x_{2} x_{5} x_{1}\right)-\frac{1}{3} W\left(x_{3} x_{5}\right) W\left(x_{2} x_{4} x_{1}\right) \\
W\left(x_{1} x_{2} x_{3} x_{4} x_{5} x_{8}\right)=\frac{1}{4} W\left(x_{1} x_{3}\right) W\left(x_{4} x_{6}\right) W\left(x_{2} x_{5}\right)+\frac{1}{4} W\left(x_{2} x_{3}\right) W\left(x_{4} x_{5}\right) W\left(x_{1} x_{6}\right) \\
-\frac{1}{4} W\left(x_{2} x_{6}\right) W\left(x_{1} x_{5}\right) W\left(x_{1} x_{3}\right)-\frac{1}{4} W\left(x_{1} x_{5}\right) W\left(x_{4} x_{6}\right) W\left(x_{2} x_{3}\right)
\end{gathered}
$$

Using these equalities it is possible to express eq.(14)-(16) as a closed algebra. It should not be difficult to discover similar algebras for coset models based on other groups. These algebras (like their local counterparts) take on their complicated, nonlinear structure due to these types of reduction identities.

In conclusion we have shown that the Poisson bracket of the gauged WZW model (eq.(6)) suggests a multi-local generalization of classical $W$ algebras.

We would like to thank E. Kiritsis for useful conversations. 


\section{Reference:}

1. A.B. Zamalodchikov, Theor, Matb. Phys. 651205 (1985). V.A. Fatsev and A.B. Zamalodchikov, Nucl. Phys. B280, 644 (1987).

2. A. Bilal, Phys. Lett. B227, 406 (1989).

3. A. Bilal, W-A Jgebra Extended Conformal Theories, Cocets and Integrable Lattice Models, Cern Preprint Cern-Th. 556i/89 (1989).

4. C.N. Pope, L.J. Romans and X. Shen, Nucl. Phys. B339, 191 (1990).

5. 1. Bakas and E. Kiritsis, Nucl. Phys. B343, 185 (1990).

6. I. Bakas and E. Kiritsis. Mod. Phys. Lett. A5, 2039 (1990).

7. G.M.T. Watts, Nucl. Phys. B328, 648 (1989); G.M.T. Watt, Nucl. Phys. B339, 117 (1990).

8. F. Bais, P. Bouwknegt, M. Surridge and K. Schoutens, Nucl. Phys. B304, 348, 371 (1988).

9. K. Bardakci, M1. Crescimanno and E. Rabinovici, Nucl. Pbys. B344, 344 (1990).

10. K. Bardakci, M. Crescimanno and S.A. Hotes, Parafermions from non-Abelian Coset Models, Berkeley Preprint UCB.PTH-90/26 (1990) 


\section{Contributions and Interest:}

This work was initiated by a comment of Prof. K. Bardakci on the fact that siace it is known that there exists a representation of certain $W$ algebras. in terms of cosets the classical structure of these theories should be understandable in term of the general Poisson bracket of the previous papers. This author's contributions include understanding the $W_{\infty}$ limit of the Poisson bracket (see eq. (7) through eq.(9)) and in computing some of the terms of the algebra in the example of su(3) of eq.(11). This author also worked on what operator would be the natural one for the non-local generalization of the W-algebras (see eq.(12) through eq.(16)). 
CHAPTER 5: Chern-Simons Qunntum Mechanics, Modular Invariance and Monopole:

Chern-Simons gauge theory is a general co-ordinate invariant field theory in threedimensions that is intimately connected with two-dimensional conformal theories. In this chapter we use Chern-Simons theory to investigate properties of the related conformal field theory.

One thing of particular interest is to show how the modular properties of the conformal field theory may be understood in term of the Chern-Simons field theory. This paper contains a construction of the space of conformal blocks at genus one, and then proceeds to use Chern-Simons theory to conpute the matricies that represent the modular transformations. Since this paper develops a realization of the operator approach, it is possible to give explicit formulae even for the general nop-abelian case. Finally, by including monopole backgrounds, some properties of the conformal model in higher genus are understood. 
Monopoles, Modular Invariance and Chern-Simona field Theory

\author{
M. Crescimanno . \\ and \\ S. A. Hotes \\ Department of Physics \\ University of California \\ Berkeley, California 94720 \\ and \\ Laurrence Berkeley Laboratory
}

to appear in Nuclear Physics B 


\section{Introduction}

Following the seminal work by Witten ${ }^{1}$, there has been sustained interest in understanding conformal field theory in terms of threndimensional Chern-Simons gauge theory. Many authors have explored the connection between the Hilbert space of the Chem-Simons gauge theory and the space of conformal blocks. One particularly illuminating formulation that has emerged from these investigations is that of the effective quantum mechanics of the Chern-Simons fieid theory $2,3,4,5$.

In other developments it has been noticed that introducing non-trivial backgrounds in a two-dimensional field theory allow one to understand novel features of the conformal field theory. For instance in ref.[6] it was shown that it is possible to infer the modular properties of a theory in higher genus by doing a genus one calculation in the presence of a monopole.

In this note we will briefly review the use of monopole backgrounds for studying conformal theories of fre fermions. We then generalize this technique to study the modular properties of the space of conformal blocks entirely from within Chern-Simons theory. Along the way we develo an explicit operator construction of the Wilson lines which become the Verlinde operators (of ref.[17] for example) in the language of conformal field theory. This extends the work of previous authors 5,4 .

The methods employed in this paper are pedestrian and the examples provided are to elucidate the properties of more complex cases to which this work trivially generalizes. In keeping with that spirit, in section II we review the case of the Ising model (or a model with any number of free fermions) in nontrivial gauge backgrounds. Section III contains 2 lightning review of what has been done with Chern-Simons quantun mechanics and is menot to acknowledge the work of a few of the many innovators of Chern-Simons quantum mechanics. It provides an introduction to the rest of the paper. Section IV combines the ideas of sections II and III for Gaussian models and section $V$ applies the techniques of 
section II to the case of arbitrary simply-laced semisimple Lie group. To dernonstrate this technique the cases of Chern-Simons theory of SU(2) and SU(3) are done in detail. Section VT is a short conclusion and discussion of results. 


\section{Monopoles, Modular Invariance and Free Fermion:}

In studying conformal theories it is often useful to imagine that the quantum degrees of freedom of the theory are coupled to external (i.e. classical background) fields. We will concentrate on the very particular case of non-trivial gauge connections. Free fermions are perhaps the very simplest of conformal theories ${ }^{7}$ and the idea of coupling their $U(1)$ current to an abelian gauge field was motivated by the coset construction of ref.[7,8,9]. The resulting theory is still naively conformal because the gauge field is introduced as a Lagrange multiplier field and has no kinetic term. In that construction one imagines that functionally integrating over the gauge field eliminates certain degrees of freedom of the free fermions and thus reduces the model of free fermions (i.e. a fermionized version of an $\mathrm{SO}(\mathrm{n}$ ) model for example) to a coset model (in the case of $S O(n)$ to the coset $S O(a) / U(1)$ ). Typically, due to the equations of motion for $\boldsymbol{A}_{\Perp}$, one considers integrating over only flat connections but if one is to take the functional integration seriously one must include contributions from non-flat connections as well.

Coupling fermions to a non-flat abelian gauge field was perhaps first studied by Landau and is of interest, for example, in understanding the quantum Hall effect (for a treatment of that problem which shares some analogy with this present work see for example ref.[10]). and has come up before in string theory ${ }^{11.12}$. Looking at the effect of monopoles directly in Chern-Simons has been explored recently in ref.[13] which has a completely different thrust than this present work. In ref.[6] an effort was made to understand how including monopole contributions modifies a model of free fermions. One finds that for fixed genus the integration over non-flat connections breaks into a sum over sectors indexed by the monopole number i,e. first Chern class, $\mathrm{N}$, of the connection. In addition to finding a new spectrum and correlation functions, sectors with different first Chern class (mod 2) had different modular properties at genus one. Indeed it was found that for those sectors in 
which $\mathbf{N}$ was evel one had the usual modular properties at genus one (here given in terms of the spin structure for the minimal modular invariant set of sections)

$$
T_{\text {Neven }}:\left(\begin{array}{ll}
+ & + \\
- & +
\end{array}\right) \rightarrow\left(\begin{array}{ll}
+ & + \\
+ & - \\
- & -
\end{array}\right)
$$

but that in sectors in which $\mathrm{N}$ was odd the behavior of the blocks under $\mathrm{T}$ were quite different:

$$
T_{\text {Nodd }:}\left(\begin{array}{l}
+ \\
+ \\
-
\end{array}\right) \rightarrow\left(\begin{array}{l}
+ \\
+ \\
-
\end{array}\right)
$$

The transformations of the blocks under $\mathrm{S}$ was found to be the same for any $\mathrm{N}$. Interestingly. although the modular properties at one loop for the $\mathrm{N}=0$ sector imply the $(+,-),(-,+),(-,-)$ sections form a modular inariant subspace, we see that including the monopole sectors suggests that this subspace of sections is not modular iavariant and that in order to urite a modular invariant theory it will be necessary to include $(t, t)$ sections as well. This is analogous to the Ising model in which at genus one the $(+,+)$ sections do not contribute but that for consistency of the model in higher genus one does need to include them.

There isre many ways to understand this result. First, were we to study the problem of free fermions in a genus $g>2$ surface $\Sigma$ we might consider concentrating on a degenerate case of the Rieman surface $\Sigma$ in which we bave a series of tori connected with thin tubes. Call $\Sigma_{1}$ just one of these tori with a piece of a single tube. In order to study operators and determinants of operators on such an object it is of course sufficient to simply specify boundary conditions for each non-trivial cycle of the torus with a point removed. Furthermore. operators involving derivatives must now contain covariant derivatives and since

$$
\frac{1}{2 \pi} \int_{\Sigma} R=2-2 g
$$

for the whole surface $\Sigma$ we know that where the tube connects to the torus there must be curvature. This means then the connection, $\omega_{\mu}$, associated with transport on the torus with 
a point removed is non-flat. Furthermose since the ieometry group of the tangent space of 2 Reimann surface is $O(2)$ the geometrical connection, $\omega_{\mu}$, may be thought of as an abelian gauge field $A_{\mu}$. Indeed "trading" $\omega_{\mu}$ for $A_{\mu}$ and thereby requiring $A_{\mu}$ to satisfy

$$
\frac{1}{2 \pi} \int_{\Sigma_{1}} R(\omega) \simeq \frac{1}{2 \pi} \int_{\text {torwe }} F(A)
$$

where $F$ is the field strenght of $A_{\mu}$, suggests that the Gauss-Bonnet result may be understood as something like a monopole quantization condition. This is also suggested by the form of the index theorem in two dimensions. Note that although in ref.[6] one always chose the flux to be uniformly distributed we will show in the appendix that concentrating the flux in one point will not change the modular properties under $T$. Thus we learn that for studying the modular properties of sections we need only specify the first Chern class of the bundle $A_{\mu}$. "Trading" $\omega_{\mu}$ for $A_{\mu}$ means that sections of operators such as the covariant derivative $\mathrm{D}$ (involving just the geometrical connection) correspond to sections associated to a charged species on the torus (geometrically flat) with monopole background. Indeed, in both cases, specifying boundary conditions will restrict one to a particular class of sections and furthermore one sees that there is a natural relation between the norms defined on the function spaces in the two cases. Using this result we may now explain why the modular properties are modified by the presence of a monopole. For simplicity imagine spreading out the abelian flux uniformly over the excirs torus (which we now think of as the plane modulo the lattice). The generator $S$ of the modita" group really only corresponds to a rotation in the plane about 3 point and a little thought indicates that jts action on sections will not be modified by the presence of an ambient magnetic field (this is shown in full detail in ref.[6]). The $T$ transiormation however really corresponds to a translation in an ambient magnetic field and thun will be associated with a residual gauge transformation.

\footnotetext{
"Strictly speaking, introducing Aux will not preacrve the holomorphic factorization of the theory but here we will be only working with one half of the theory anyway and so do not have to concern ourselves with this point further.
} 
In the case of add monopole charge this will result in a nontrivial phase contribution to the transport, i.e. the boundary conditions of the sections will change. Let's make this concrete in a picture. Denote the sides of the torus as $\mathrm{Cl}$ and $\mathrm{C2}$ (as shown in Fig. 1), and denote the boundary condition of a given section by $(A, B)$ where generally $A$ and $B$ are non-abelian phases (or group elements) but for the case at hand take the values +1 or -1 . of course, when there is no flux one finds for $T$ and $S$

$$
T:(A, B)-\left(A, B A^{-1}\right) ; \quad S:(A, B) \rightarrow\left(B, A^{-1}\right)
$$

which leads to the usual transformation described in eq.(2.1). The natural way to think about $T$ in this context is that in the new torus after performing $T$ the new cycle C2' (see Fig. 1) is composed of the original $\mathrm{C2}$ followed by the $\mathrm{Cl}$ cycle and so to find out how transport on the new torus compares with the old one we rnust simply compose the respective transports. The fact that this results in $A^{-1} B$ instead of $A B$ is really just the difference between viewing the transformation as passjue rather than active on the transport. Here we choose it as in eq.(2.5) to be consistent with the convention of most authors. Note that this convention gives, as abstract group elements $S^{2}=\mathrm{C}$, the charge conjugation operator and $(S T)^{3}=1$. Now let's consider what happens when one includes the background gauge field of a monopole. As seen from the figure, the transport along C2' is no longer simply determined by the composition of the transport along $\mathrm{C2}$ then $\mathrm{Cl}$. In addition to the composition of transport along those cycles there is an additional phase due to the flux inside the triangle $\mathrm{C2C1C2}$ '. Since for connections with unit monopole charge the transport around the entire torus is trivial, the additional phase acquired by transport along cycle C2' relative to that of $\mathrm{C2}$ is -1 (because the triangle C2C1C2' has exactly half the area of the torus). Fo. those who feel uncomfortable with this analysis because of the use of "uniform flux" we refer to the full result of the appendix which demonstrates that for any flux distribution the additional phase under $T$ is as described above. Thus with this 
additional phase we find that eq.(2.5) is modified

$$
T:(A, B) \rightarrow\left(A,-B A^{-1}\right) ; \quad S:(A, B) \rightarrow\left(B, A^{-1}\right)
$$

Note that throughout $S$ is unchanged. Eq.(2.6) yields the relations of eq.(2.2). Notice that with larger monopole charges the above argument implies that for even monopole charge one has the behavior of eq.(2.1) and for odd monopole charge one finds the behavior of eq.(2.2). Again, since the $\mathrm{N}=1$ monopole corresponds naturally to a torus with a single tube at tached (see eq.(2.4)) which is related to the boundary of moduli space for a genus two surface, we rediscover the bit of string theory lore that if one satisfies the modular intariance through to second loop then the theory is modular invariant for arhitrary genus. Thus modular properties of the conformal blocks of a theory in higher genus may be understood by using monopole backgrounds in genus one.

Another way to understand this property is through the fusion algebra of a theory of free fermions. Take for simplicity the case of a single real fermion, i.e. the Ising model. One knows that in the Ising model there are exactly three primary fields namely $1, \psi$ and $\sigma$ of conformal dimension $0,1 / 2$ and $1 / 16$ respectively. These fields correspond to linear combinations of the $(-,-),(+,-),(-,+)$ sectors. Note that these three fields form a modular covariant set at one loop. The $(t, t)$ sector bas a zero mode (i.e. constant sections) and so does not contribute to the partition function at genus 1 . However were we to imagine constructing a two-loop amplitude by using the fusion rules the natural object to study would be the one point functions on the torus. There is a non-trivial one point function on the torus that involves the insertion of the $\psi$. In order to see why this one point function doesn't vanish it is necessary to include in the path integral the contributions to this amplitude coming from the $(t, t)$ sector ${ }^{\dagger}$. Simply said, the zero mode is absorbed by the insertion of the $\psi$ and thus the $(+,+)$ sector that was formerly not included on the

\footnotetext{
'The authors are indebted to $\mathrm{E}$. Kiritais for a elarification of this point.
} 
grounds that it was zero at genus one is needed to make the two loop calculation modular invariant. Now, were we to construct Green's functions on the torus with a monopole background we would find that, as a consequence of the index theorem, the only non-zero Green's functions involve an "additional" insertion of the fermion. This means that every non-zero Green's function has as a first approximation the same modular properties as the zero modes. That this behavior persista to all orders is proven in ref.[6] in which it was shown that all modes (zero and non-zero modes) have the same modular properties. Thus it is not a surprise that we learn about the modular properties of the theory in higher loop by studying the theory in the background of a monopole. 


\section{Chern-Simons Quantum Mechenie.}

Let us now briefly review the development of the effective quantum mechanics of CheraSimons theory. As described by Witten' ${ }^{1}$ the Bilbert space of Chern-Simons theory is isomorphic to the space of conformal blocks of the underlying conformal field theory. This identification follows from studying the degrees of freedom of the Chern-Simons theory after gauge fixing, and understanding the action of the residual general co-ordinate invariance of the theory in terms of conformal transformations. This was expanded upon in several very readable papers for the case in which the spatial part of the 3-manifold is a torus ${ }^{2.3 .4 .5}$. In essence these works suggest that, choosing the axial gauge $\left(A_{0}=0\right)$, one should first satisfy the constraints classically (which are that the non-abelian flux vanishes everywhere os the 2-manifold) and then proceed to enforce the quantum mechanical commutators on the operators associated with the observables. As shown in the above references, the observables are the nontrivial Wilson lines. For a torus (without a point removed) the Wilson lines are the traces of the holonomies around the two non-trivial cycles of the torus. As before we label the holonomies by $A$ and $B$. These are the path-ordered exponentials of the gauge fields along the paths $\mathrm{C} 1$ and $\mathrm{C} 2$ respectively. Then the classical constraint that the flux vanish reads,

$$
A B A^{-1} B^{-1}=I
$$

where $I$ is the identity. As was done in the above references, we satisfy tuis constraint by taking $A$ and $B$ to be in the maximal torus of the group and without loss of geserality may also take the gauge field to be constant and in the Cartan sub-algebra. Let

$$
A=e^{i a+r} ; \quad B=e^{i \beta \cdot t}
$$

where the $\tau$ 's are geverators of the Cartan sub-algebra. Now one implements the canoniral 
commutation relations implicit in the Chern-Simons theory and finds

$$
\left[\alpha^{i}, \beta^{j}\right]=\frac{2 \pi i}{k} \delta^{i j} \Rightarrow A B A^{-1} B^{-1}=\xi^{n} I
$$

where

$$
\xi^{k}=1
$$

and where $n$ is a number that depends on the normalization of the generators and $k$ is the coeficient in front of the action. This is the naive result. This relation is amended in the non-abelian case in that $k$ is shifted to $k+c$ where $c$ is the quadratic casimir in the adjoint. These points are discussed at length from the point of view of Chern-Simons theory in ref. $[4,19,20,22,23]$ and is understood from the two-dimensional action functional approach in a non-perturbative way in ref.[14,15]. At any rate, we save the complexities of the non-abelian case for later. For the abelian case we have $n=1$ and it is easy to see that we may represent $A$ and $B$ in the above algebra in terms of $k$-dimensional matrices.

For completeness we compute the $S$ and $T$ matrices of the abelian Chern-Simons theory. This is often called the Gaussian model because it is equivalent to a free compactified boson. Although $T$ and $S$ are computed in ref.[4,16], we wish to display ther here as finite dimensional matrices on the space of conformal blocks because these matrices. will be useful for comparison later when we develop the theory for the case of the non-trivial backgrounds. Viewing $T$ and $S$ as residual general co-ordinate transformations we now write how the components of the vector field $A_{\mu}$ transform,

$$
T:(\alpha, \beta) \rightarrow(\alpha, \beta-\alpha) ; \quad S:(\alpha, \beta) \rightarrow(\beta,-\alpha) .
$$

In terms of the holonomies we find,

$$
T:(A, B) \rightarrow\left(A, r B A^{-1}\right) ; \quad S:(A, B) \rightarrow\left(B, A^{-1}\right),
$$

where

$$
r^{2}=\xi
$$


Because these are symmetries of the underlying conformal model we expect that $T$ and $S$ may be represented in terms of unitary transformations on the $k$-dimensional subspace of the conformal blocks. In the basis where $A$ is taken to be diagonal (and $B$ is thus a raising operator) we find

$$
T_{l j}=a \xi^{-\rho / 2} \delta_{l j ;} \quad S_{l j}=\frac{b}{\sqrt{k}} \xi^{e \ell j}
$$

where $a$ is a phase that is to be determined in terms of the central charge of the conformal theory (see for exainple [16]) and the phase in $S$ called $b$ may be partially fixed by requiring that the $S$ and $T$ form a representation of the modular group, namely that as before $S^{2}=$ $\mathrm{C}$ and $(S T)^{3}=I$. Without loss of generality we take $b=1$. Again note that $S^{2}$ is only equal to $I$ for $k=1$ and $k=2$. 


\section{Monopoles in Abelian Chern-Simons Theory}

Having developed some intuition about monopole backgrounds and knowing the formulation of the Gaussian model, we now explore some features of this model in higher loop by using monopole backgrounds. We begin by studying abelian Chern-Simons theory on a manifold with topology $R \times \Sigma_{1}$ winere $\Sigma_{1}$ is 2 torus with a point removeci. If we consider only trivial sections of the abeliar: line bundle over $\Sigma_{1}$ we recover the case studied in the last section (the flux is zero everywhere on the torus, even at the point removed.) Instead consider non-trivial sections of the abelian line bundle. We implement this as follows. Imagine holding fixed a classical background gauge field $A_{\mu}^{e}$ that is the gauge field of a monopole whose flux is entirely concentrated at the missing point, and then use Chern-Simons theory to study the behavior of the quantum fluctuations about that background. For cnnvenience we restrict the quantum fluctuations to be in a trivial bundle i.e. we allow for no non-trivial operator insertions at the point removed. This restriction wakes the problem solvable but. as described in section II, unfortunately makes the connection of this technique with the one-point functions indirect. Also note that the analogy between geometrical connection and gauge field described in section II is not so direct because we have started with a theory that was metric-independent and so we should generalize the earlier argument to this case. Furthermore we suspect that it is necessary to include the sectors with non-triviai bundles for the theory to have well a well defined cluster decomposition. With these points in mind we write the total $A_{\mu}$ for the abelian case 25

$$
A_{\mu}=A_{\mu}^{c}+A_{\mu}^{q}
$$

This may be simply generalized to the non-abelian case by taking $A_{\mu}=A_{\mu}^{q}+U^{-1} A_{\mu}^{c} U$ where $U$ is just the path-ordered exponential of the $A_{\mu}^{q}$, a fat connection with trivial homotopy: That this is a consistent decomposition of $A_{\mu}$ may be seen by writing out the field strength $F$, for this $A_{\mu}$ or just recognizing that if $A_{\mu}^{q}$ was a pure gauge (which is true classically) 
$A_{\mu}$ and $A_{\mu}^{c}$ would be gauge transforms of one another. At any race, when considering nontrivial backgrounds we will always simplify the problem by puting the $A_{\mu}^{c}$ in the same Cartan sub-algebra as $A_{\mu}^{q}$ and so the decomposition of $A_{\mu}$ will indeed become linear. Aiso note that when this decomposition eq.(4.1) is substituted into the action functional, and $A_{\mu}^{c}$ is treated as a classical fieid, that the commutator of the $A_{\mu}^{q}$ components is the same as in eq.(3.3). Furthermore, as far as quantization of the monopole is concerned, for the abelian case we imagine working with $U(1)$ compact. As usual the non-abelian case takes care of itself because parh-ordered exponentials are charged and thus defining monopole charge in the non-abelian case is unambiguous. The monopole quantization condition is of course the same for every genus. For an excellent review of monopoles the reader is referred to the monograph of Coleman ${ }^{18}$. A little thought convinces one that the moduli space of the $A_{\mu}^{q}$ is again (for the torus) two-dimensionel and that eq. (3.1) holds with $A$ and $B$ the path-ordered exponentials of the full $A_{\mu}$ of eq.(4.1). Notice that although $A_{\mu \mu}^{c}$ is not single valued on the torus, because of the quantization condition the holonomies $A$ and $B$ are well defined.

A]though the operator algebras with and without the monopole look identical, they difier in their behavior under modular transformations. As explained in the appendix. and as discussed in section II, the monopole introduces an additional phase in the definition of $T$ on the operators $A$ and $B$. We find that for the case of odd monopole charge we again have

$$
T_{\text {Nodd }}:(A, B) \rightarrow\left(A,-r B A^{-1}\right) ; \quad S:(A, B) \rightarrow\left(B, A^{-1}\right) .
$$

Notice that $T_{\text {Nodd }}$ is different than the $T$ in the case of even monopole charge given in eq.(3.6). As 2 matrix on the space of states $T_{N o d d}$ is (compare with the $T$ of eq. $(3.8)$ )

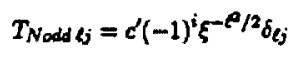

Notice that this new $\boldsymbol{T}$ is still diagonal and that the vacuum-vacuum matrix element 
(given by $T_{\infty}$ ) is unchanged. In general we expect that adding the monopoles does not change the central charge of the theory and one shouldn't take the -1 's as a sign that the conformal dimensions of certain fields have jumped in the presence of the monopole. After all, we are including the nontrivial monopole sector to study the behavior of $T$ and $S$ in higher genus and so the conformal dimensions are not necessarily equal to the phases of the diagonal elements of $T$. At any rate we do learn the amusing fact that $S$ and $T_{\text {Nodd }}$ do, in general, form only a projective representation of the modular group. By "projective" we mean that $(S T)^{3}=d I$ where $d$ is some phase that cannot be removed by unitary transformations of the operators. For the abelian case at even $k$ this is detailed in Table 1. The case of $k$ odd is more difficult to characterize and will be commented on later. ,

\begin{tabular}{|c|r|}
\hline$k$ & $d$ \\
\hline 2 & $-\mathrm{i}$ \\
4 & -1 \\
6 & $\mathrm{i}$ \\
8 & 1 \\
10 & $-\mathrm{i}$ \\
12 & -1 \\
14 & $\mathrm{i}$ \\
16 & 1 \\
\hline
\end{tabular}

Table 1: the abelian case, even $k$

Note that computation suggests $(S T)^{3}=(-i)^{k / 2} T$.

The fact that the monopole in this Gaussian model acts the same as in the Ising model case (introducing a -1 phase in the $T$ action and neatly falling into classes of odd and even monopole charge), and the four-fold periodicity of the projective representation of the modular group in the Gaussian model, at this point seem mysterious. They will in fact 
be neatly understood from the operator construction of the SU(2) Chern-Simons theory discussed in the next section. 


\section{Monopolea in Non-Abelinn Chern-Simone Theory}

We now discuss some generalities of the operator approuch to Chern-Simons effective quantum mechanics for a non-abelian gauge group. The motivation for much of the discussion comes from ref. $[4,19]$.

Geaeralization of the simple techniques uned above for the abelian case to the nonabelian case is not completely obvious. First, although the commutator of the two components of the gauge field naively looks the same as above, one knows from general conformal fieid theory results that the relevent parameter for the deecription of the conformal blocks is not $k$ but $k+c$ where $c$ is the quadratic casimir in the adjoint representation (normalized so $c=\mathrm{n}$ for $\mathrm{SU}(\mathrm{n}))$. Furthermore, the conformal blocks are indexed over a specific set of the integral representations, which are a set of fundamental weights all displaced from the origin by $\rho$ which is half the sum of the positive roots $4,19,20,22,23$. It is not completely clear how these shifts are to be implemented in the Chera-Simons effective quantum mechanics. Here we present an unambiguous and pedestrian implementation of these shifts in a manner which makes sense of the Verlinde operators entirely in the context of Chern-Sirnons field theory.

In short we find that one may represent the individual elements of the path-ordered exponentials as products of operators of Gaussian models. This is vaguely reminiscent of the conformal field theory lore that ultimately conformal theories are theories of free fields with unusual statistics. Although we represent the terms in the Wilson line by products of Gaussian models the theory is not a simple product of Gaussian models. Instead, enforcing gauge invariance of the Wilson line and modular invariance will result in a truncation of the Bilbert space of the product of the Gaussian models that will, in a seace, give the resulting theories :heir non-trivial statistics.

We first represent the Wilson line operators on the trucated Eilbert space. We must 
then relate this to the gauge invariant observables of the Chern-Simons field theory. Since the observables are already in the torus of the group, the only residual gauge transformations are those associated with the Weyl action. We find that requiring the observables to be gauge invariant is equivalent to representing them on a subspace of states, each state of which is odd under all involutions of the Weyl group. Since $T$ and $S$ commute with the action of the Weyl group, these states form a mudular invariant subspace. This will all be made explicit in the examples below. Finally let us remark that after finding these states one will be left with a natural way of interpreting the shift $\lambda \rightarrow \lambda+\rho$. The construction of $T$ and $S$ for the non-abelian case has been outlined in ref.[4]. This reference has a simple abstract formula for $T$ and $S$ and is unclear about the actual representation of $T$ and $S$ as matrices on a finite dimensional Hilbert space. This is due perhaps in part to the fact that there is confusion about actually understanding the Wilson line as a regulated quantum mechanical operator ${ }^{19}$. Below we construct an operator approach to the observables in Chern-Simons effective quantum mechanics and will explicitly construct the $T$ and $S$ matrices of the conformal theory entirely within the context of Chern-Simons gauge theory. This will be necessary for understanding how the non-abelian monopoles change the modular properties of the non-abelian theory. Once this operator construction is completed we obtain the $T$ and $S$ matrices for any monopole background.

The fundamental group of the torus has two generators, $\mathrm{C} 1$ and $\mathrm{C} 2$. The only gauge invariant quantities of the theory are the traces of the Wilson lines about these two loops. Again, classically these Wilson lines must commute since $\mathrm{Cl} \cdot \mathrm{C} 2$ is homotopically equivalent to $\mathrm{C2} . \mathrm{Cl}$. As described earlier, this implies that we may require the gauge field $A_{\mu}$ to take values in the Cartan sub-algebra. Define the algebra elements

$$
\begin{aligned}
& a(t)=a_{i}(t) \nu^{j}=\int_{C_{1}} d x^{\mu} A_{\mu^{j}}^{j} \nu^{j} \\
& b(t)=b_{j}(t) \nu^{j}=\int_{C_{2}} d x^{\mu} A_{\mu}^{j} \nu^{j}
\end{aligned}
$$

where the $\nu_{i}(i=1, \ldots, r ; r=\operatorname{rank}(G))$ are the simple roots. The components along the 
diagonal of the path-ordered exponentials are then given by products of operators of the type,

$$
A_{i}(t)=e^{i a_{1}(t)} ; \quad B_{i}(t)=e^{i b_{1}(t)} .
$$

With these variables, the effective action takes the form

$$
S=\frac{k+c}{2 \pi} \int d t a_{i}(t) C_{\mathrm{ij}} \dot{b}_{\mathrm{j}}(t)
$$

where $C_{i j}^{\prime}=\left\langle\nu_{i} \mid \nu_{j}\right\rangle$ is the Cartan matrix. This action is linear in time derivatives and so give the following C.C.R.

$$
\begin{gathered}
{\left[a_{i}, b_{j}\right]=-\frac{2 \pi i}{k+c}\left(C^{-1}\right)_{i j}} \\
A_{i} B_{j} A_{i}^{-1} B_{j}^{-1}=\exp \left(\frac{2 m i}{k+c}\left(C^{-1}\right)_{i j}\right)
\end{gathered} .
$$

To realize these commutation relations, ws diagonalize the $r$ commuting $A_{i}$ matrices. Their eigenvalues for a given state will label the porition of that state in the weight lattice. The $B_{i}$ matrices will act as raising operators along the $r$ primary weight vectors.

Let's now explicitly study the case $\mathrm{G}=\mathrm{SU}(2)$. The the rank is one and the quadratic Casimir $c=2$. The Cartan matrix is just a number ( 2 in our convention) which implies that $C^{-1} i j=1 / 2$. Thus the Gaussian model we use to represent the elements of the pathordered exponential bas $2(k+2)$ states. Figure 2 contains a diagram of the lattice of integral weights of the model for $k=4$.

For convegience of exposition define $k^{\prime}=2(k+2)$. The $T$ and $S$ af the $k^{\prime}$-dimensional Gaunian model is a deacribed in eq.(3.8) in which $k$ is replaced with $k^{\prime}$. We now implement gauge invariance by using the Weyl group, $W$, to break this space of states into Weyl covariant aubepacen. The key recison why this is a ensible thing to do is that Weyl transformation always commute with $T$ and $S$ (since one corresponds to gauge tranformations and the other correspond to co-ordinate transformations they commute classically in the Chern-Simons lagrangian and thus here are taken to commute quantum mechanically) and 10 constructing the characters of the Weyl action in the Gaussian Hilbert space will 
automatic. Hy yield modular invariant and Weyl covariant subspaces. Thus representing the algebra of observables on these subspaces will correspond to implementing gauge invariance. In the case of $\mathrm{SU}(2)$ level $k$ there are two subspaces

Odd under $W_{i}|i>-| k^{\prime}-i>$ for $0<i<k^{\prime} / 2$

Even under $W ; \quad|0>,| k^{\prime} / 2>,|i\rangle+\mid k^{\prime}-i>$ for $0<i<k^{\prime} / 2$

Now we may implement the shift $\lambda \rightarrow \lambda+\rho$ by realizing that ( corresponds to the state $i=1$ and so this state should be thought of as the "shifted vacuum". We can thus rule opt relating the states even under $\mathrm{W}$ to those of $\mathrm{SU}(2)$ level $k$ because they mix with $|0\rangle$ which. if $\mid 1>$ is to be the new vacuum, $\mid 0>$ has negative relative conformal dimension. The states which are odd under $\mathrm{W}$ form a modular invariant subspace and are the correct ones for realizing the $\mathrm{SC}^{\prime}(2)$ level $k$ model. Indeed, they correspond to spins $i<k / 2$. W'riting the $T$ and $S$ of the Gaussian model on this subspace we find the expected result

$$
T_{\ell j}=\exp \left(-\frac{i \pi}{4}+\frac{i \pi \ell^{2}}{2(k+2)}\right) \delta_{\ell ; i} \quad S_{\ell j}=\sqrt{\frac{2}{k+2}} \sin \left(\frac{\ell j \pi}{k+2}\right)
$$

Note that as expected the ceatral charge is $3 k / k+2$.

We now consider what happens when we include monopole backgrounds on the worldsheet. In SU(2) there are strictly speaking no topological monopoles since the group is homotopically trivial. Instead, were we to consider the group $\mathrm{SU}(2) / Z_{2}=\mathrm{SO}(3)$ we would have two distinct monopole sectors. It is not difficult to see that in terms of the operators above, the monopoles in this case simply modify $T$ exactly as in eq. (4.3) of the Gaussian model (the two monopcle sectors in the $\mathrm{SU}(2) / Z_{2}$ model correspond to charge even and tharge odd in the abelian case). It is well known that the $\mathrm{SU}(2) / Z_{2}$ model is only weil defined for $k=0 \bmod (4)$ and the periodicity of the projective representation found (sits Table 2) is suggestive of that fact*. Furthermore, including the monopole modifies the $T$.

\footnotetext{
'The authore thank K. Intrilijgator for a discuesion of this point.
} 
eigenvalues of those states of the $\mathrm{SU}(2)$ model that are contained in the $\mathrm{SU}(2) / Z_{2}$ model all in the same way. This is a phenomena we will see occur in the $\mathrm{SU}(3) / Z_{3}$ model, and is probably generic. We feel it is suggestive of the fact that these models are modular invariant in higher genus but clearly much work remains to be done on this. We will comment further on these observations later.

\begin{tabular}{|c|r|}
\hline$k$ & $d$ \\
\hline 1 & $-\mathrm{i}$ \\
2 & 1 \\
3 & $-\mathrm{i}$ \\
4 & -1 \\
5 & $\mathrm{i}$ \\
6 & 1 \\
7 & $-\mathrm{i}$ \\
8 & 1 \\
\hline
\end{tabular}

Table 2: $\quad \mathrm{SU}(2)$ level $k$

Tote computation suggests $(S T)^{3}=-(-i)^{k} I$.

Sext we consider the group $\mathrm{G}=\mathrm{SU}(3)$ at level $k$. We have $c=3$ and $\mathrm{G}$ is rank 2 . The Cartan matrix is,

$$
C=\left(\begin{array}{cc}
2 & -1 \\
-1 & 2
\end{array}\right) ; \quad C^{-1}=\frac{1}{3}\left(\begin{array}{ll}
2 & 1 \\
1 & 2
\end{array}\right) .
$$

Let $\xi$ be a primitive root of unity of order $3(k+c)$, i.e. $\xi^{3(k+c)}=1$. We have,

$$
\begin{aligned}
& A_{1} B_{1} A_{1}^{-1} B_{1}^{-1}=\xi^{2} \\
& A_{2} B_{2} A_{2}^{-1} B_{2}^{-1}=\xi^{2} \\
& A_{1} B_{2} A_{1}^{-1} B_{2}^{-1}=\xi \\
& A_{2} B_{1} A_{2}^{-1} B_{1}^{-1}=\xi
\end{aligned} .
$$


Now define the basis $\psi_{i j}$ such that

$$
\begin{aligned}
& A_{1} \psi_{i j}=\xi^{i} \psi_{i j} \\
& A_{2} \psi_{i j}=\xi^{j} \psi_{i j}
\end{aligned}
$$

The commutation relations of eq.(5.7) give

$$
\begin{aligned}
& B_{1} \psi_{i j}=\psi_{i+2, j+1} \\
& B_{2} \psi_{i j}=\psi_{i+1, j+2}
\end{aligned}
$$

We thus need keep only the connected sector of states $\psi_{i j}$ that satisfy the condition $i+j=$ 0 mod(3). Implementing periodicity of the canonical variables leaves a finite set of states (the dimensionality of which is $3(k+c)^{2}$ ). We have thus created a fundamental cell of $\left(\Lambda_{W} /(k+c) \Lambda_{R}\right)$ where $\Lambda_{W}$ is the weight lattice of $G$ and $\Lambda_{R}$ is the root lattice.

The next step is to calculate the $S$ and $T$ operators of this Gaussian model. To accomplish this, we again implement

$$
T:(A, B) \rightarrow\left(A, r B A^{-1}\right) ; \quad S:(A, B) \rightarrow\left(B, A^{-1}\right)
$$

where $r^{2}=\xi$. We find,

$$
\begin{gathered}
T_{i j, k \ell}=\delta_{i k} \delta_{\mathrm{j} \ell} \xi^{\frac{1}{3}\left(i^{2}+j^{2}-i j\right)} \\
S_{i j, k \ell}=\left\langle\psi_{i j}|S| \psi_{k \ell}>=\frac{1}{\sqrt{3(k+c)^{2}}} \xi^{-\left[3(i k+j l)-\frac{1}{3}(i l+j k)\right]}\right.
\end{gathered}
$$

These matrices satisfy the defining relations of $\mathrm{SL}(2, \mathrm{Z})$.

Finally, we need to implement the anomalous shift $\lambda \rightarrow \lambda+\rho$ where $\rho$ is one half the sum of the positive roots. Thus $\rho$ indicaties the new vacuum state (conformal dimension zero). We first identify the Weyl orbits of $\left(\Lambda_{W} /(k+c) \Lambda_{R}\right)$. As expected we find that the number of orbits with length equal to the order of the Weyl group is equal to the number of modular invariant states in the theory. The general formula for $\mathrm{SU}(n)$ is,

$$
\frac{(n+k-1) !}{(n-1) !(k) !}
$$


We identify each Weyl orbit of maximal length with a different primary field. We then construct a modular invariant sector by choosing one linear combination of states from each of these special Weyl orbits. To achieve this we employ the identity (i.e. $T$ and $S$ again commute with Weyl transformations)

$$
\left.\left.<\psi^{\prime}|S, T| \psi\right\rangle=<w \psi^{\prime}|S, T| w \psi\right\rangle
$$

where $w$. is an arbitrary element of the Weyl group. For SU(3) the orbits are of length 1. 3 and 6 . To reduce $T$ and $S$ to the modular invariant sector, we choose coefficients for the six states of a given orbit of length six to be a nontrivial character of the Weyl group. This necessarily will be orthogonal to states in the orbits of shorter length. For $k=1$ the resulting 3-dimensional vector space is in fact modular invariani. We now give explicit details.

The Weyl group of $\operatorname{SL}(3)$ is generated by the reflections $w_{1}$ and $w_{2}$. As unitary matrices on the basis given above we have,

$$
\left(w_{1}\right)_{i j, k \ell}=\delta_{j, \ell} \delta_{i, \ell-k i} . \quad\left(w_{2}\right)_{i j, k \ell}=\delta_{i, k} \delta_{j, k-\ell} .
$$

There are three Weyl orbits of order $\operatorname{six}$ in $\left(\Lambda_{W} /(k+c) \Lambda_{R}\right)$ at $k+c=4$. These are shoun below in Figure 3. In Figure 3 states related by the Weyl group are labelled with the same letter. Note that thert: are $15=6 ! / 2$ ! 4 ! different Weyl orbits. The three orbits of order 6 are labelled $a, b, c$. Each maximal orbit is isomorphic under the Wnyl group, with the action shown below in Figure 4. If we choose the cocficients (also defined by $w_{i} \psi=-\psi: \mathrm{i}=$ 1,2) given in the diagram for each of the three sets of states, we obtain a modular intariant subspace, with the following $S$ and $T$ matrices (the ordering of the basis is a,b,c)

$$
S=\left(\begin{array}{ccc}
1 & 1 & 1 \\
1 & \eta^{2} & \eta \\
1 & \eta & \eta^{2}
\end{array}\right) ; \quad T=\left(\begin{array}{ccc}
1 & 0 & 0 \\
0 & \eta & 0 \\
0 & 0 & \eta
\end{array}\right) ; \quad \text { with } \eta^{3}=1
$$


These satisfy the defining relations of $\operatorname{SL}(2, \mathrm{Z})$. The effect of a classical monopole backgrounds is easily calculated using the above techniques with the simple result

$$
T_{\mathrm{n}}=\left(\begin{array}{ccc}
1 & 0 & 0 \\
0 & \eta^{1+2 u} & 0 \\
0 & 0 & \eta^{1+a}
\end{array}\right) ; \quad \text { with } \eta^{3}=1
$$

where the monopole number a runs over the set $0,1,2\left(E Z_{3}\right)$. It is simple to show that $\left(S T_{n}\right)^{3}$ is proportional to the identity for the three choices of $\mathrm{a}$. Note that $T$ and $S$ are similar to the $T$ and $S$ of the Gausian model at (odd) $k=3$ but that here it is simple to characterize the sectors with monopoles. Also note that the only state whose $T$ eigenvalues doesn't change with the inclusion of the monopoles is the state in the $\mathrm{SU}(3) / Z_{3}$ model. 


\section{V1. Conclusion}

We have shown the modular properties of a conformal field theory at genus one change in the presence of a monopole in a way that is indicative of the modular properties in higher genus. Furthermore with an explicit operator construction of the algebra of observables in Chern-Simons theory we have used monopoles to provide insight into the center-moded models of ref.[24,25]. Work under way includes understanding the orbifold constructions (for example see ref.[26]) from the point of view of Chern-Simons theory with classical backgrounds and better understanding the connection between monopole backgrounds and the general theory in higher genus. 


\section{Acknowledgements}

We wish to thank K. Bardakci for guidance and belpful conversations, M. Bos, A. Giveon, K. Intrilligator, D. Bar-Natan, A. Polychronakos, I. Bars, P. Woit, and E. Kiritsis for interesting comments related to this work. One of us (MJC) wishes to thank the I.C.T.P. in Trieste for hospitality during the early stages of this work. This work was supported in part by the Deptartment of Education graduate student fellowship (MJC), by the Director, Office of Energy Research, Office of High Energy and Nuclear Physics of the U.S. Department of Energy under Contract DE-AC03-76SF00098 and in part by the National Science Foundation under grant PHY85-1585T. 


\section{Appendix}

In this appendix we show that the r.udular properties of the conformal blocks under $T$ depend only on the first Chern class of the gauge bundle and not on the distribution of the flux. We simply compute the line integrais along C1 and C2 (see Fig. 1) of an arbitrary flux distribution and will find the behavior under $T$ to be that described in the text. As usual, the gauge field for the monopole, $A_{\mu}^{c}$, bas been gauge transformed into the Cartar sub-aigebra and therefore it will be enough to consider the abelian case. Furthermore we ignore possible additional classical holonomy. Note that since $A_{\mu}^{c}$ is not periodic we expect that we will not be able to represent it as a theta function on the torus. Instead we do a full analytic calculation of the line integrals in a non-holomorpl ic gauge. In Coloumb gauge,

$$
\partial_{\mu} A^{c \mu}=0
$$

we may solve the equation,

$$
F=\frac{1}{2} \epsilon^{\mu \nu} F_{\mu \nu}=m \delta^{(2)}\left(x-x_{0}\right)=m \delta^{(2)}\left(x^{\prime}, y^{\prime}\right)
$$

in the plane and find for $A_{\mu}^{c}$,

$$
A_{1}^{\varepsilon}=\frac{m y^{\prime}}{x^{\prime 2}+y^{\prime 2}+\varepsilon} ; \quad A_{2}^{\varepsilon}=\frac{-m x^{\prime}}{x^{\prime 2}+y^{\prime 2}+\varepsilon} .
$$

where the $\varepsilon$ bas been added to regulate the singularity at $x^{\prime}=y^{\prime}=0$, the position of the flux. Now we gauge transform this result to $A^{c}{ }_{2}=0$ gauge. This will be a convenient gauge for compiting the line integrals after we dice up the plane into tori. At any rate, in the plane in thi: gauge we have

$$
A_{1}^{c}=\frac{m y^{\prime}}{x^{\prime 2}+y^{\prime 2}+\varepsilon}-\partial_{x}\left[\frac{-m x^{\prime}}{\sqrt{x^{\prime 2}+\varepsilon}} \text { atan }\left(\frac{\left|y^{\prime}\right|}{\sqrt{x^{\prime 2}+\varepsilon}}\right)\right]+\partial_{x} f ; \quad A_{2}^{f}=0
$$

where $f$ is a function of $x$ only and will be determined by physical grounds that will be described shortly. 
Now we imagine drawing in this plane the lattice generated by two discrete translations where we, without loss of generality, take one translation to be $z \rightarrow z+1$ and, per usual, the other to be generated by $z \rightarrow z+\tau$ where $\tau=c+i d$. In order to now bave an gauge field that is still not periodic but respects the symmetries of the gauge field of a monopole on a torus we imagine solving eq.(A.2) for a delta function in each cell of the lattice. Since we are interested only in whether the line integrals of $A_{\mu}^{c}$ are dependant on the distribution of the flux we restrict ourselves to computing them. Simply translating and summing the $A_{\mu}^{e}$ we have above will not lead to a well defined sum and so we regulate it in the following natural way. In the chosen gauge eq.(A.4) we see that the integral along sides 2 and 4 (see Fig 5) are indeed equal and that therefore the condition on the total flux is given by

$$
\int_{3} A^{c}-\int_{1} A^{c}=2 \pi m
$$

*here $m$ is some fixed number which represents the flux at $x_{0}$ and is not necessarily an i.i.eger. Actually computing this from an $A_{\mu}^{e}$ found by performing a lattice sum over individual translated $A_{\mu}^{c}$ 's of eq.(A.4) tells us how to regulate the sum by converting it to a line integral from $-\infty$ to $+\infty$ with a single source and then systematically including the other poles in the plane in a consistent way. . One finds that in eq.(A.5) only the last two terms of $A^{c}{ }_{1}$ contribute and that the integral of the term involving $f$ contributes exactly half of the right hand side of eq.(A.5). Now in computing the line integral along path 2 in the same way as for eq.(A.5) one finds that the contributions from the first two terms cancel and that one is left just with the contribution from the $f$ term. Since $f$ is only a function of $x$ and since the path has advanced only $c$ units per cell to the right we have

$$
\int_{2} A^{c}=\pi m c
$$

Now note that this result did not depend on where one placed the flux. Also, since this line integral occurs in an exponent, one finds that under $T$ (in which $c$ goes to $c+1$ ) the new 
phase acquired by the exponential is.

$$
\text { additional phase }=e^{i \pi m} \text {, }
$$

and now summing up contributions from many different fluxes at various locations so that the total fux, $m$, is integer (i.e. requiring monopole quantization) we find the additional phase acquired under $T$ is precisely that described in the text. Note also that $T$ will in general move the flux around but will not change the first Chern number (the total monopole charge) of $A_{\mu}^{c}$. 


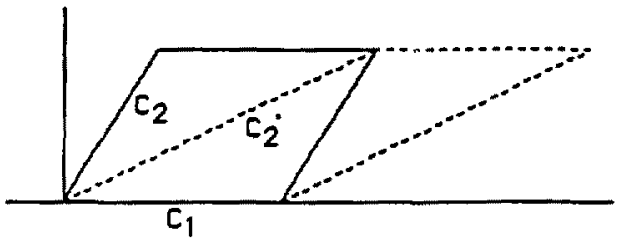

Figure 1

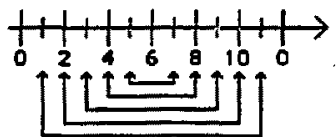

Figure 2: Arrows indicate states realated by Weyl group 


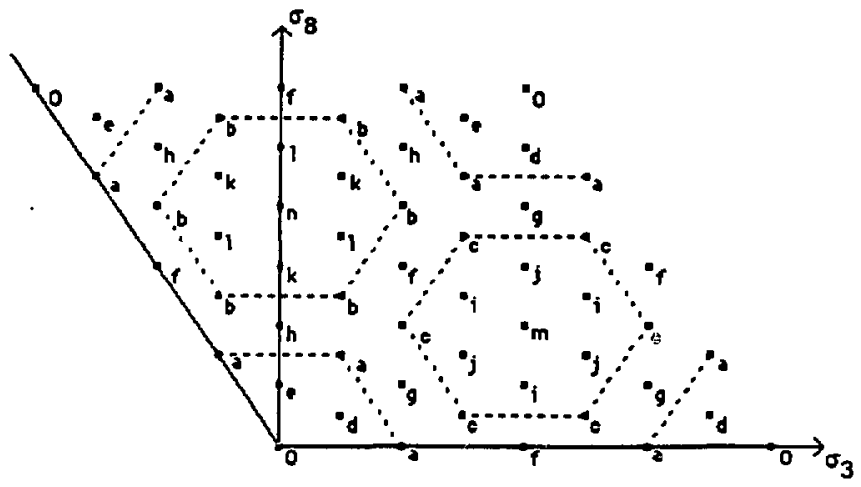

Figure 3: Weyl orbits of SU(3) level 1 Gaussian model
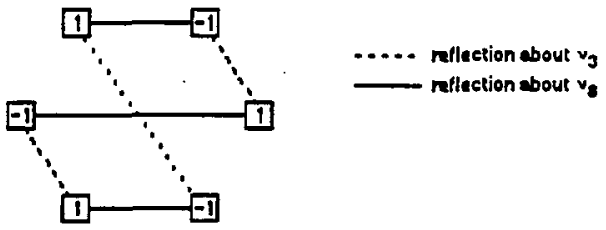

Figure 4: The action of the Weyl group on an orbit of maximal onder. The Numbers in the boxes correspond to coefficients used to form the single state 


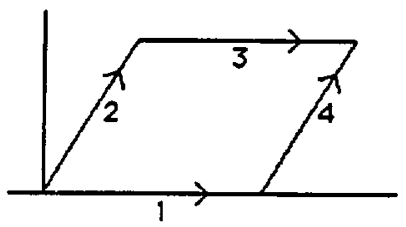

Figure 5 


\section{Bibliography}

1. E. Witten, Comm. Math. Phys. 121, 351 (1989).

2. M. Bos and V.P. Nair lnt. Journ. Mod. Phys. A5, 959 (1990).

3. M. Bos and V.P. Nair, Phys. Lett. B223, 61 (1989).

4. S. Elitzur, G. Moore, A. Schwimmer, and N. Seiberg, Nucl. Phys. B326, 108 (1989).

5. A. P. Polychronakos, Florida Preprint UFIFT-BEP-89-9, and Ann. Phys. 203, 231 (1990).

6. K. Bardakci and. M. Crescimanno, Nucl. Phys. B313, 269 (1989).

7. K. Bardakci and M. Halpern Phys. Rev. D3, 2493 (1971).

8. P. Goddard, A. Kent, and D. Olive, Phys. Lett. B152, 88 (1985); Comm. Math. Pbys. 103, 105 (1986).

9. E.T. Tomboulis, Phys. Lett. B188, 165 (1987).

10. F.D.M. Haldane and E.H. Rezayi, Phys. Rev. B31, 2529 (1985).

11. B.A. Ovrut and S. Thomas, Phys. Rev. D43, 1314 (1991).

12. A. Abouelsaood, C.G. Callan, C.R. Nappi and S.A. Yost, Nucl. Phys. B280, 599 (1987).

13. I. Afleck, J. Harvey, L. Palla and G. Semenoff, Nucl. Phys. B328, 575 (1989).

14. A.M. Polyakov and P.B. Wiegmann, Phys. Lett. B141, 223 (1984).

15. A.M. Polyakov and P.B. Wiegmann, Phys. Lett. B131, 121 (1983).

16. E. Verlinde, Nucl. Phys. B300, 360 (1988). 
17. H. Verlinde and E. Verlinde, Preprint PUPT 89/1149 (1989).

18. S. Coleman, The Magnetic Monopole Fifty Years Later. Based on lectures given at the School of Sub-Nuclear Physics "Ettore Majorana" (1981).

19. M. Bos, privata communication.

20. L. Alvarez-Gaume, J.M.F. Labastida, A.V. Ramallo, Nucl. Phys. B334, 103 (1990).

2i. R.D. Pisarski and S. Rao, Phys. Rev. D22, 2081 (1985).

22. W. Chen, G.W. Semenoff and Y. Wu, Preprint 89-0956(UTAF) and Mod. Phys. Lett. A5. 1833 (1990).

23. D. Gepner and E. Witten, Nucl. Phys. B278, 493 (1986).

24. G. Moore and X. Seiberg, Phy's. Lett. B220, 422 (1989).

25. R. Dijkgraaf, E. Verlinde and H. Verlinde, Comm. Math. Phys. 115, 649 (1988): R. Dijkgraaf, C. Vafa, E. Verlinde and H. Verlinde, Comm. Math. Phys. 123, 485 (1989). 


\section{Contributions and Interests}

This author initiated this project and was motivated by the intriguing connection between conformal field theory and Chern-Simons theory that was pointed out by Witten in ref.[1]. The bulk of that which is new in this paper, with the exeption of the pleasingly general formulation of eq.(5.1) and eq.(5.3) and some of the details of the SU(3) case (both of which were done by S.A. Bates), is this author's contribution. As of late there has been additional work done on underatanding the observeables as operators on the Hilbert space constructed here. A simple picture of the fusion rules and the Verlinde conjecture emerges. This will appear in the new version of the paper to be published.

Open questions of interest are, for example, how to prove the Verlinde conjecture from this point of view and whether one might understand the geometrical structure behind coset constructions. These and other questions are currently under scrutiny. 


\section{CHAPTER 6: Conclusions and Outlook}

Although each of the preceding chapters have concluding sections, here we wish to point out some of the unifying themes and results of this work and also to connect this work to some of the recent investigations by other authors.

One underlying idea of this work is understanding Wilson lines as quantum mechanical operators. In typical higher-dimensional gauge theories trying to understand the Wilson lines as quantum mechanical operators is a troublesome task. However in lowdimensional gauge theories of the type studied here (both the two-dimensional WessZumino-Witten model and Chern-Gimons field theory) one has an infinite-dimensional symmetry group which acts to organize the physical degrees of freedom of the theory and possibly allow one to understand the W'ilson line as a quantum mechanical operator. Indeed, recently Alekseev, et.al. have understood the Wilson lines of particularly simple conformad theories as quantum operators by using a lattice regularization. ${ }^{1}$. Tot suprisingly the Wilson lines form a realization of a quantum group. In their approach they start with Poisson brackets that are very similar to that of eq.(5.16) of Chapter II, section a. It seems that a promising approach for further study would be to find a continuum regularization of the Wilson line.

Interestingly enough, one may study the particularly simple conformal theories investigated by ref.[1] as Chern-Simons field theories. Then, as shown in chapter $V$, it is possible to understand the Wilson lines quantum mechanically at least for the torus without introducing a lattice regularization or indeed any regulation at all! Unfort unately this approach is not $2 s$ general as the approach of ref.[1] and much work remains to be done to if it is profitable to use techniques from Chern-Simons theory to understand the quantum mechanics of Wilson lines for a general situation. 
Another theme of this work has been to try to understand conformal models from - lagrangian point of view. Here of particular interest is understanding the gaugedWZW model as a type of non-linear $\sigma$ model. Becently Mandial et.al ${ }^{2}$ and Witten ${ }^{3}$ bave tried to understand what string propagation on various two-dimensional backgrounds is like by stadying cosets of $s l_{2}$ WZW model. As would be expected, the resulting lagrangian is very similar to that of eq.(6.7) chapter III, section a. It would be interesting to pursue the two-dimensional spacetime interpretation of various coset models to get a glimpse at the nature of stringy gravity.

Low-dimensional field theories, although an old subject that has been intensely studied, continues to be a rich lesting-ground for some of the most interesing questions and intriguing ideas of physics. This author feels that much is yet to be learned by the continued vigorous efforts of many researchers in this topic. 


\section{Biblivgraphy}

(a) A. Alekseev, L. Faddeev, ard A. Volkov, The Unravelling of the Quantun Group Structure in the WZNW Theory, Preprint CERN-TH-5981/91

(b) G. Mandal, A.M. Sengupta and S. Wadia, Classical Solutions of Two-Dimensiona] String Theory, Preprint IASNS-HEP-91/10

(c) E. Witten, On String Theory and Black Holes, Preprint IASNS-HEP-91/12 